\title{
Synopsis of the
}

Parasites of

Vertebrates

of Canada

Helminths and Protozoa of Terrestrial Mammals

Murray J. Kennedy

Alberta Agriculture

Veterinary Laboratory

6909 - 116 Street

Edmonton, Alberta

Canada T6H 4P2 
Copies of this publication are available from the Queen's Printer, 11510 Kingsway Avenue, Edmonton T5G 2Y5

Orders must be accompanied by a cheque for $\$ 5.00$ made out to The Provincial Treasurer. 


\title{
SYNOPSIS OF THE PARASITES OF VERTEBRATES OF CANADA
}

Helminths and Protozoa of Terrestrial Mammals

\author{
Murray J. Kennedy \\ Alberta Agriculture \\ Veterinary Laboratory \\ 6909 - 116 Street \\ Edmonton, Alberta \\ Canada T6H 4P2
}


Digitized by the Internet Archive in 2016

https://archive.org/details/synopsisofparasi00kenn_1 
This synopsis contains Parasite-Host and Host-Parasite lists of 355 species of parasites from 92 species (15 with 1 subspecies; 9 with 2 subspecies; 3 with 3 subspecies; and 1 with 4 subspecies) of terrestrial mammals from Canada. The 355 species of parasites are distributed among higher taxa as follows: Protozoa 70; Trematoda - 62; Cestoidea - 65 ( Cysticercus spp. are not included in the total); Nematoda - 158; and Acanthocephala - 2. 



\section{Introduction}

The compiled information covers the period from 1853 to June, 1985 and was extracted from numerous publications in the primary scientific literature. Journal series such as the Canadian Journal of Zoology, Canadian Journal of Research, Journal of Wildlife Diseases, Journal of Protozoology, Proceeding of the Helminthological Society of Washington, and the Journal of Parasitology were searched in their entirety. Abstracting series such as Zoological Records, Helminthological Abstracts, and Protozoology Abstracts were scanned for additional references. Theses and reports of federal and provincial governments and of other institutions were not searched extensively, hence, some existing records may have been omitted.

In providing references to the parasite-host-distribution data, I have attempted to include only reports based on original records, but screening all reports to ensure complete exclusion of repetitive records based on the same material was not possible. Publications that do not provide original parasite-host-distribution records but deal with some aspect of Canadian mammalian wildlife parasitology are compiled in a supplementary list of references.

The synopsis indicates that up to June, 1985, at least 355 species of parasites have been reported from 92 species (15 with 1 subspecies; 9 with 2 subspecies; 3 with 3 subspecies; and 1 with 4 subspecies) of terrestrial mammals. Parasites not identified to the species level are not included in the total number. The 355 species are divided among major taxa as follows: Protozoa - 70; Trematoda - 62; Cestoidea - 65 (Cysticercus spp. are not included in the total); Nematoda - 158; and Acanthocephala - 2.

The taxonomic arrangement adopted for each phylum or class of parasitic organism included in Canadian records, in most cases, is as follows:

Protozoa - Levine et al. (1980)

Trematoda - Brooks et al. (1985)

Cestoidea - Schmidt (1986)

Nematoda - Anderson et al. (1974-1983)

Acanthocephala - Amin (1982)

Host nomenclature and classification conforms to that of Hall (1981), except that Odocoileus rather than Dama is used for the generic name of the white-tailed, mule, and black-tailed deer. The entries in the synopsis are organized in accordance with the format adopted by Margolis and Arthur (1979) in their Synopsis of the parasites of fishes of Canada.

The Parasite-Host List is organized by higher taxa of parasites in the following order: Protozoa, Platyhelminthes (Trematoda, Cestoidea), Aschelminthes (Nematoda), and Acanthocephala. Species, genera, and family names are listed in alphabetical order within their respective next higher taxon, which in the case of a family is either a superfamily or an order, depending on the group in question and the usefulness of including the superfamily or order category.

For each parasite species listed, the following information is provided:

1) The current scientific name, including author(s) and date(s), followed by any recognized synonyms that may have been used in establishing the Canadian record(s). No attempt has been made to evaluate systematically the validity of published records, but attention is drawn to obvious errors. Adult and larval stages are recorded separately for the Cestoidea and Nematoda. The term metacestode is used either when the Canadian record used the term, or in cases where the type of cestode larva reported is uncertain. 
2) The site of occurrence of the parasite in its host(s) (aberrant sites usually are not included). When the site was not given, the likely site was determined from other records.

3) The hosts. Only currently accepted scientific names are given in the Parasite-Host List. Numbers in parentheses after each host name correspond with the numbers assigned to the references establishing the particular parasite-host records.

4) Distribution ( Dist.) Species distribution is indicated by Provincial or Territory boundaries listed in alphabetical order as follows: Alta (Alberta), BC (British Columbia), Man (Manitoba), NB (New Brunswick), Nfld (Newfoundland), NS (Nova Scotia), NWT (Northwest Territories), Ont (Ontario), PEI (Prince Edward Island), Que (Quebec), Sask (Saskatchewan), and YT (Yukon Territory).

5) Authors responsible for the records are listed in chronological order of the published records. To enable the reader to grasp at a glance the author(s) responsible for particular parasite-host-locality records, I numbered the references listed under "Records" and placed the corresponding number(s) after the host name, and each reference is followed by the locality or localities from which the parasite was (were) reported. An interrogation mark (?) after the locality indicates an uncertain locality record. When only one host is listed for a particular parasite, the references are not numbered, and when all records are from the same locality, the latter was not listed after the authors' names.

6) Under remarks, explanatory comments, as required, are offered on systematics, nomenclature, misidentifications, and synonyms of parasites; and on the hosts, sites within hosts, and validity of previous records.

In the Host-Parasite List, within each order or suborder, the families, genera, species, and subspecies are listed alphabetically. Host synonyms included are only those that were used in establishing the Canadian parasite records. After the name of each parasite species, its geographic distribution in the host in question is given in parentheses.

The REFERENCES are given in two groups, the first includes those directly referred to in the text and a second, SUPPLEMENTARY REFERENCES, contains a list of publications dealing with various aspects of parasites of Canadian mammals but which do not contain original parasite-host records.

To facilitate access to information in the parasite-host and host-parasite lists, the publication concludes with an INDEX to parasite and host scientific names. 


\section{PARASITE-HOST LIST}




\section{KINGDOM ANIMALIA SUBKINGDOM PROTOZOA}

\section{PHYLUM \\ SARCOMASTIGOPHORA SUBPHYLUM MASTIGOPHORA \\ CLASS ZOOMASTIGOPHOREA ORDER DIPLOMONADIDA}

\author{
FAMILY HEXAMITIDAE
}

Giardia mesocricetus Filice, 1952

Site: intestine

Hosts:Mesocricetus auratus $(1,2)$

Lab. rats (2)

Dist.: Ont

Records: 1. Grant and Woo 1978a, 2. 1978b

Giardia microti Kofoid and Christiansen, 1915

Site: intestine

Hosts:Mesocricetus auratus (3)

Microtus pennsylvanicus $(2,3)$

Field mice (1)

Lab. hamsters (3)

Dist.: NS, Ont

Records: 1. Simon 1921 (NS); 2. Grant and

Woo 1978a (Ont), 3. 1978b exp. (Ont)

Giardia muris Grassi, 1881

Site: intestine

Hosts: Mesocricetus auratus (4)

Rattus norvegicus $(2,3,4)$

Lab. mice $(1,3,4)$

Lab. hamster (4)

Lab. rats (1)

Dist.: NS, Ont, Que

Records: 1. Simon 1921 exp. (NS); 2. Firlotte 1948 (Que); 3. Grant and Woo 1978a (Ont), 4. $1978 \mathrm{~b}$ exp. (Ont)

Giardia peromysci Filice, 1952

Site: intestine

Host: Peromyscus maniculatus

Dist.: Ont

Records: Grant and Woo 1978a, 1978b exp.
Giardia simoni Lavier, 1924

Site: intestine

Hosts:Rattus norvegicus $(1,2)$

Lab. rats $(1,2)$

Dist.: Ont

Records: 1. Grant and Woo 1978a, 2. 1978b exp.

\section{ORDER KINETOPLASTIDA}

Trypanosoma citelli Watson, 1912

Site: blood

Host: Spermophilus richardsonii

Dist.: Alta

Record: Watson and Hadwen 1912

Trypanosoma evotomys Hadwen, 1912

Site: blood

Hosts: Clethrionomys gapperi Myotis yumanensis saturatus Rabbits

Dist.: $\mathrm{BC}$

Record: Watson and Hadwen 1912

Trypanosoma hedricki Bower and Woo, 1981

Site: blood

Host: Eptesicus fuscus

Dist.: Ont

Records: Bower and Woo 1981; Paterson and Woo 1984 exp.

Trypanosoma leporis- sylvaticus Watson, 1912

Site: blood

Host: Sylvilagus nuttallii

Dist.: Alta

Record: Watson and Hadwen 1912

Trypanosoma lewisi (Kent, 1880) LaVeran and Mesnil, 1901

Site: blood

Host: Rattus sp.

Dist.: Ont

Record: Watson and Hadwen 1912 
Trypanosoma microti Laveran and Pettit, 1909

Site: blood

Hosts:Microtus pennsylvanicus

Microtus sp. lab (exp.)

Dist.: Ont

Record: Woo et al. 1980

Trypanosoma myoti Bower and Woo, 1981

Site: blood

Host: Myotis lucifugus

Dist.: Ont

Records: Bower and Woo 1981; Paterson and

Woo 1984 exp.

Trypanosoma otospermophili (Wellman and

Wherry, 1910) Laveran, 1911

Site: blood

Hosts:Spermophilus columbianus $(1,2)$

S. franklinii $(1,2)$

S. parryii $(1,2)$

$S$. richardsonii $(1,2)$

S. tridecemlineatus $(1,2)$

Dist.: Alta, YT

Records: 1. Hilton and Mahrt 1972a, 2. 1972b

Trypanosoma peromysci Watson, 1912

Site: blood

Hosts:Peromyscus maniculatus

P. m. nebracensis

Dist.: Alta

Record: Watson and Hadwen 1912

Trypanosoma soricis Hadwen, 1912

Site: blood

Hosts:Blarina brevicauda (2)

Sorex vagrans (1)

Dist.: BC, Ont

Records: 1. Watson and Hadwen 1912 (BC);

2. Woo et al. 1980 (Ont)

Trypanosoma tamiasi Riedel, 1975

Sites: blood and organs

Hosts: Eutamias minimus (1)

Tamias striatus $(1,2)$

Dist.: Ont

Records: 1 . Riedel 1975; 2. Woo et al. 1980

Trypanosoma sp.

Site: blood

Host: Squirrel

Dist.: BC

Record: Watson and Hadwen 1912

ORDER RETORTAMONADIDA
Chilomastix bettencourti da Fonseca, 1915

Site: intestine

Host: Rattus norvegicus

Dist.: Que

Record: Firlotte 1948

ORDER TRICHOMONADIDA

Tritrichomonas muris (Grassi, 1879)

Syn.: Trichomonas muris

Site: intestine

Host: Rattus norvegicus

Dist.: Que

Record: Firlotte 1948

SUBPHYLUM SARCODINA

CLASS LOBOSEA

ORDER AMOEBIDA

Entamoeba muris (Grassi, 1879)

Site: intestine

Host: Rattus norvegicus

Dist.: Que

Record: Firlotte 1948

Unidentified Amoebae

Site: intestine

Hosts: Rangifer tarandus caribou

Dist.: BC

Record: Low 1976

PHYLUM APICOMPLEXA

CLASS SPOROZOEA

ORDER EUCOCCIDIIDA

SUBORDER EIMERIINA

Besnoitia spp.

Sites: skin, skeletal muscle, nasal mucosa, eye, pituitary, lymph nodes

Hosts: Rangifer tarandus (1)

R. . caribou (3)

Barren-ground caribou (2)

Dist.: NWT, Sask

Records: 1. Gibbs 1960 (NWT?); 2. Choquette et al. 1967(NWT); 3. Wobeser 1976 (Sask)

Eimeria ahsata Honess, 1942

Site: intestine

Host: Ovis c. canadensis

Dist.: Alta, BC

Record: Uhazy et al. 1971 
Eimeria albertensis Hair and Mahrt, 1970

Site: intestine

Host: Ursus americanus

Dist.: Alta

Record: Hair and Mahrt 1970

Eimeria arizonensis Levine, Ivens, and

Kruidenier, 1957

Site: intestine

Host: Peromyscus maniculatus

Dist.: BC

Record: Levine and Ivens 1963

Eimeria athabascensis Samoil and Samuel, 1977

Site: intestine

Host: Lepus americanus

Dist.: Alta

Record: Samoil and Samuel 1977b

Eimeria banffensis Lepp, Todd and Samuel,1973

Site: intestine

Host: Ochotona collaris

Dist.: Alta, YT

Record: Hobbs and Samuel 1974

Eimeria barretti Lepp, Todd, and Samuel, 1972

Site: intestine

Hosts: Ochotona collaris (2)

$$
O \text {. princeps }(1,2)
$$

Dist.: Alta, YT

Records: 1. Lepp et al. 1972 (Alta); 2. Hobbs and Samuel 1974 (Alta, YT)

Eimeria bilamellata Henry, 1932

Site: intestine

Hosts:Spermophilus columbianus

S. franklinii

S. richardsonii

Dist.: Alta

Record: Hilton and Mahrt 1971

Eimeria borealis Hair and Mahrt, 1970

Site: intestine

Host: Ursus americanus

Dist.: Alta

Record: Hair and Mahrt 1970

Eimeria calentinei Duszynski and Brunson, 1973

Site: intestine

Hosts: Ochotona collaris (2)

$$
O \text {. princeps }(1,2)
$$

Dist.: Alta, YT

Records: 1. Lepp et al. 1973 (Alta); 2. Hobbs and Samuel 1974 (Alta, YT)
Eimeria callospermophili Henry, 1932

Site: intestine

Hosts:Spermophilus columbianus
S. franklinii
S. richardsoni

Dist.: Alta

Record: Hilton and Mahrt 1971

Eimeria carinii Pinto, 1926

Site: intestine

Hosts:Rattus norvegicus

Dist.: Que

Record: Firlotte 1948

Eimeria circumborealis Hobbs and Samuel, 1974

Site: intestine

Hosts:Ochotona collaris

O. princeps

Dist.: Alta, YT

Record: Hobbs and Samuel 1974

Eimeria confusa Joseph, 1969

Site: intestine

Hosts:Sciurus carolinensis

Dist.: Ont

Record: Lee and Dorney 1971

Eimeria crandallis Honess, 1942

Site: intestine

Host: Ovis c. canadensis

Dist.: Alta, BC

Record: Uhazy et al. 1971

Eimeria dorneyi Levine and Ivens, 1965

Site: intestine

Host: Glaucomys sabrinus

Dist.: Ont

Record: Soon and Dorney 1969a

Eimeria faurei (Moussu and Marotel, 1902)

Martin, 1909

Site: intestine

Hosts: Ovibos moschatus (2)

Ovis c. canadensis (1)

Dist.: Alta, NWT, Que

Records: 1. Uhazy et al. 1971 (Alta); 2.

Duszynski et al. 1977 (Alta, NWT, Que)

Eimeria granulosa Christensen, 1938

Site: intestine

Hosts: Ovibos moschatus (2)

Ovis c. canadensis (1)

Dist.: Alta, NWT

Records: 1. Uhazy et al. 1971 (Alta); 2.

Duszynski et al. 1977 (NWT) 
Eimeria holmesi Samoil and Samuel, 1977

Site: intestine

Host: Lepus americanus

Dist.: Alta

Record: Samoil and Samuel, 1977b

Eimeria intricata Spiegl, 1925

Site: intestine

Host: Ovis c. canadensis

Dist.: Alta

Record: Uhazy et al. 1971

Eimeria keithi Samoil and Samuel, 1977

Site: intestine

Host: Lepus americanus

Dist.: Alta

Record: Samoil and Samuel 1977b

Eimeria klondikensis Hobbs and Samuel, 1974

Site: intestine

Hosts: Ochotona collaris

O. princeps

Dist.: Alta, YT

Record: Hobbs and Samuel 1974

Eimeria lateralis Levine, Ivens, and Kruidenier, 1957

Site: intestine

Hosts:Spermophilus columbianus

S. richardsonii

Dist.: Alta

Record: Hilton and Mahrt 1971

Eimeria leporis Nieschulz, 1923

Site: small intestine

Hosts:Lepus americanus

Dist.: Alta

Record: Samoil and Samuel 1977b

Eimeria miyairii Ohira, 1912

Site: intestine

Host: Rattus norvegicus

Dist.: Que

Record: Firlotte 1948

Eimeria moschati Duszynski, Samuel, and Gray, 1977

Site: intestine

Host: Ovibos moschatus

Dist.: Alta, NWT, Que

Record: 1. Duszynski et al. 1977
Eimeria mustelae Kingscote, 1934

Site: intestine

Hosts:Mustela putoris (exp)

M. vison

Dist.: Ont

Record: Kingscote 1934

Eimeria ninakohlyakimovae Yakimoff and

Rastegaieff, 1930

Site: intestine

Hosts:Ovis c. canadensis

Dist.: Alta, BC

Record: Uhazy et al. 1971

Eimeria ontarioensis Lee and Dorney, 1971

Site: intestine

Host: Sciurus carolinensis

Dist.: Ont

Record: Lee and Dorney 1971

Eimeria oomingmakensis Duszynski, Samuel, and Gray, 1977

Site: intestine

Host: Ovibos moschatus

Dist.: NWT

Record: Duszynski et al. 1977

Eimeria ovibovis Duszynski, Samuel, and Gray, 1977

Site: intestine

Host: Ovibos moschatus

Dist.: Alta, NWT, Que

Record: Duszynski et al. 1977

Eimeria ovina Levine and Ivens, 1970

Site: intestine

Hosts:Ovibos moschatus (2)

Ovis c. canadensis (1)

Dist.: Alta, BC, NWT, Que

Records: 1. Uhazy et al. 1971 (Alta, BC); 2.

Duszynski et al. 1977 (Alta, NWT, Que)

Eimeria parva Kotlan, Mocsy, and Vajda, 1929

Site: intestine

Host: Ovis c. canadensis

Dist.: Alta, BC

Record: Uhazy et al. 1971

Eimeria princepsis Duszynski and Brunson, 1973

Site: intestine

Hosts: Ochotona collaris

O. princeps

Dist.: Alta, YT

Record: Hobbs and Samuel 1974 
Eimeria robertsoni (Madsen, 1938) Carvalho, 1943

Syn: Eimeria magna var. robertsoni

Madsen, 1938

E. m. f. townsendi Carvalho, 1943 pro parte

E. perforans var. groenlandica

Madsen, 1938.

Site: duodenum

Host: Lepus americanus

Dist.: Alta

Records: Samoil and Samuel 1977a exp., 1977b

Eimeria rochesterensis Samoil and Samuel, 1977

Site: intestine

Host: Lepus americanus

Dist.: Alta

Record: Samoil and Samuel 1977b

Eimeria rowani Samoil and Samuel, 1977

Site: intestine

Host: Lepus americanus

Dist.: Alta

Record: Samoil and Samuel 1977b

Eimeria ruficaudati Gill and Ray, 1960

Site: intestine

Host: Lepus americanus

Dist.: Alta

Record: Samoil and Samuel 1977b

Eimeria separata Becker and Hall, 1931

Site: intestine

Host: Rattus norvegicus

Dist.: Que

Record: Firlotte 1948

Eimeria siniffi Levine and Ivens, 1963

Site: intestine

Host: Peromyscus maniculatus

Dist.: BC

Record: Levine and Ivens 1963

Eimeria spermophili Hilton and Mahrt, 1971

Site: intestine

Hosts:Spermophilus franklinii

S. richardsonii

Dist.: Alta

Record: Hilton and Mahrt 1971

Eimeria stiedae Lindemann, 1865

Site: liver

Host: Ondatra zibethicus

Dist.: Ont

Record: Law and Kennedy 1932
Eimeria tamiasciuri Levine, Ivens, and

Kruidenier, 1957

Site: intestine

Host: Tamiasciurus hudsonicus

Dist.: Alta

Record: Mahrt and Chai 1970, 1972.

Eimeria toddi Dorney, 1962

Site: intestine

Host: Tamiasciurus hudsonicus

Dist.: Alta

Record: Mahrt and Chai 1970, 1972

Eimeria townsendi (Carvalho, 1943) Pellerdy, 1956

Syn: Eimeria magna f. townsendi Carvalho, 1956 pro parte

Eimeria magna Perard, 1925 pro parte

Site: intestine

Host: Lepus americanus

Dist.: Alta

Record: Samoil and Samuel 1977b

Eimeria worleyi Lepp, Todd, and Samuel, 1972

Site: intestine

Host: Ochotona princeps

Dist.: Alta

Record: Lepp et al. 1972

Eimeria spp.

Site: intestine

Hosts:Lepus americanus (1)

Ovibos moschatus (4)

Ovis canadensis californiana (2)

Sciurus carolinensis (3)

Dist.: Alta, BC, Man, NWT, Ont, Que

Records: 1. Boughton 1932 (Man); 2. Blood 1963 (BC); 3. Lee and Dorner 1971 (Ont); 4.

Samuel and Gray 1974 (Alta capt., NWT,

Que capt.)

Remarks: Boughton (1932) believed his

Eimeria spp. may be Eimeria stiedae and E. perforans.

Frenkelia microti (Findlay and Middleton, 1934)

Biocca, 1968

Site: brain

Host: Ondatra zibethicus

Dist.: Ont

Record: Karstad 1963 
Isospora marquardti Duszynski and Brunson, 1972

Site: intestine

Hosts: Ochotona collaris

O. princeps

Dist.: Alta, YT

Record: Hobbs and Samuel 1974

Isospora yukonensis Hobbs and Samuel, 1974

Site: intestine

Host: Ochotona collaris

Dist.: YT

Record: Hobbs and Samuel 1974

Sarcocystis campestris Cawthorn, Wobeser and Gajadhar, 1983

Sites: intestine, vascular endothelium,

skeletal muscle

Hosts:Spermophilus richardsonii (exp.)

Taxidea taxus

Dist.: Sask

Record: Cawthorn et al. 1983

Sarcocystis spp. cysts

Site: cardiac muscle, skeletal muscle

Hosts: Alces alces $(2,3,4,10)$

A. alces andersoni (7)

Bison bison $(1,10)$

Cervus elaphus (10)

Odocoileus hemionus (10)

O. virginianus $(6,10)$

Oreamnos americanus (10)

Ovibos moschatus (8)

Ovis canadensis (10)

Rangifer tarandus (5)

R. t. caribou (9)

Dist.: Alta, NS, NWT, Ont, Sask

Records: 1. Cameron 1923 (Alta); 2. DeVos and Allin 1949 (Ont); 3. Kelly et al. 1950 (NS); 4. Peterson 1955 (Ont); 5. Gibbs 1960 (NWT?); 6. Karstad and Trainer 1969 (Ont); 7. Barrett 1972 (Alta); 8. Samuel and Gray 1974 (NWT); 9. Wobeser 1976 (Sask); 10. Mahrt and Colwell 1980 (Alta)
Toxoplasma gondii Nicolle and Manceaux, 1908

Site: serum

Hosts:Blarina brevicauda (1)

Canis latrans $(1,2)$

Martes martes (2)

$M$. pennanti (2)

Mephitis mephitis $(1,2,4)$

Mus musculus (3)

Mustela vison (2)

Peromyscus maniculatus (3)

Procyon lotor (2)

Rattus norvegicus (3)

Sylvilagus floridanus (2)

Ursus americanus $(1,2)$

Vulpes vulpes $(1,2)$

Dist.: Alta, Ont, Sask

Records: 1. Quinn et al. 1976 (Ont); 2. Tizard et al. 1976 (Ont); 3. Tizard et al. 1978 (Ont);

4. Schowalter et al. 1980 (Alta, Sask)

Remarks: All reports used serological methods to detect $T$. gondii.

Unidentified Eucoccidiida

Eucoccidiida gen. spp.

Site: epididymis, intestine, liver

Hosts:Alopex lagopus (4)

Cervus elaphus (5)

Ochotona princeps (1)

Ursus americanus (3)

Rabbit (2)

Dist.: Alta, NWT, Ont, Que, Sask

Records: 1. Lubinsky 1957 (Alta); 2. Westermann and Engelbert 1969 (Ont); 3. Juniper 1978 (Que); 4. Eaton and Secord 1979 (NWT); 5 . Hrudka et al. 1983 (Sask)

\section{SUBORDER ADELEINA}

Hepatozoon spp.

Site: lungs

Hosts: Mustela vison (2)

Tamiasciurus hudsonicus (1)

Dist.: Alta, Ont

Records: 1. Mahrt and Chai 1972 (Alta); 2.

Presidente and Karstad 1975 (Ont). 
PHYLUM MICROSPORA

CLASS MICROSPOREA

ORDER MICROSPORIDA

Encephalitozoon cuniculi Levaditi, Nicolau and Schoen, 1923

Site: brain

Host: Ondatrazibethicus

Dist.: Sask

Record: Wobeser and Schuh 1979
Unidentified Protozoa

Protozoa gen. spp.

Site: intestine

Host: Ondatra zibethicus

Dist.: Man

Record: McKenzie and Welch 1979

\section{SUBKINGDOM EUMETAZOA}

\section{PHYLUM \\ PLATYHELMINTHES \\ CLASS TREMATODA \\ SUBCLASS DIGENEA}

FAMILY BRACHYLAEMIDAE

Brachylaima sp.

Site: intestine

Host: Microtus pennsylvanicus terraenovae

Dist.: Nfld

Record: Rausch 1952b

Postharmostomum helicis (Leidy, 1847) Robinson, 1949

Site: caecum

Host: Peromyscus maniculatus

Dist.: Que

Record: Schad 1956

Scaphiostomum pancreaticum McIntosh, 1934

Site: pancreatic duct

Host: Tamias striatus

Dist.: Ont

Record: Jensen 1972 exp.

\section{FAMILY DICROCOELIIDAE}

Dicrocoelium dendriticum (Rudolphi, 1819) Looss, 1899

Syn: Dicrocoelium lanceolatum

Sites: liver, gall bladder, pancreatic duct, duodenum

Hosts: Lepus americanus struthopus (2) Mink (1)

Dist.: Nfld, PEI

Records: 1. Conklin and Baker 1930 (PEI); 2.

Dodds and Mackiewicz 1961 (Nfld)

\section{FAMILY DIPLOSTOMIDAE}

Alaria alata (Goeze, 1782) Krause, 1914

Site: intestine

Host: Vulpes vulpes

Dist.: Man, PEI, Que

Record: Swales 1933b (PEI, Que); Baron 1970 (Man)

Alaria americana Hall and Wigdor, 1918

Syn.: Alariacanis

Site: intestine

Hosts: Canis latrans $(4,5)$

C. lupus $(1,4,5,6,7)$

Lynx lynx (4)

L. rufus (4)

Martes pennanti (4)

Ursus americanus (7)

Vulpes vulpes $(1,2,3,4,8)$

Dist.: Alta, NB, NS, Ont, PEI, Que, YT

Records: 1. Law and Kennedy 1932 (Ont); 2.

Swales 1933b (PEI, Que); 3.Pearson 1954 exp.

(Ont), 4. 1956 (Ont); 5. Holmes and Podesta

1968 (Alta); 6. Choquette, Gibson et al. 1973

(YT); 7. Addison et al. 1978 (Ont); 8. Smith $1978 b$ (NB, NS)

Alaria americana Hall and Wigdor, 1918

(mesocercaria)

Sites: many tissues

Hosts: Lutra canadensis (2 nat.)

Mus musculus (2)

Mustela putorius (2)

Peromyscus maniculatus $(1,2)$

Ferret (1)

Lab. mice (1)

Dist.: Ont

Records: 1. Pearson 1954 exp., 2. 1956 exp. 
Alaria arisaemoides Augustine and Uribe, 1927

Site: intestine

Hosts: Canis latrans $(3,4,6)$

C. lupus $(5,6)$

Vulpes vulpes $(1,2,6,7)$

Dist.: Alta, Man, NB, NS, NWT, Ont, YT

Records: 1. Law and Kennedy 1932 (Ont); 2.

Pearson 1954 exp. (Ont); 3. 1956 (Ont); 4.

Holmes and Podesta 1968 (Alta); 5. Choquette, Gibson et al. 1973 (NWT, YT); 6. Samuel, Ramalingam et al. 1978 (Man); 7. Smith 1978b (NB, NS)

Alaria arisaemoides Augustine and Uribe, 1927 (mesocercaria)

Sites: many tissues

Hosts: Mus musculus (2)

Peromyscus maniculatus $(1,2)$

Ferret (1)

Lab. mice (1)

Dist.: Ont

Records: 1. Pearson 1954 exp., 2. 1956

Alaria canadensis Webster and Wolfgang, 1956

Site: small intestine

Host: Mephitis mephitis

Dist.: Que

Record: Webster and Wolfgang 1956

Alaria craterum (Barker and Noll, 1915)

Site: duodenum

Hosts: Mustela vison (1)

Ondatra zibethicus (1)

Dist.: Ont

Record: Law and Kennedy 1932

Alaria marcianae (LaRue, 1917) Walton, 1950

Site: intestine

Hosts: Canis latrans (1)

C. lupus $(1,3)$

Procyon lotor hirtus (2)

Vulpes vulpes (2)

Dist.: Man, Que, Sask

Records: 1. Samuel, Ramalingam et al. 1978

(Man); 2. Hoberg and McGee 1982 (Sask); 3.

McNeill et al. 1984 (Que)
Alaria mustelae Bosma, 1931

Site: duodenum, intestine

Hosts: Martes pennanti (4)

Mustela vison (1)

Ondatra zibethicus $(1,2,5)$

Vulpes vulpes $(1,3)$

Dist.: Man, NB, NS, Ont

Records: 1. Law and Kennedy 1932 (Ont); 2.

Sweatman 1952b (Ont); 3. Smith 1978b (NB,

NS); 4. Dick and Leonard 1979 (Man); 5.

McKenzie and Welch 1979 (Man)

Alaria mustelae Bosma, 1931

(mesocercaria)

Site: lungs, spleen, musculature

Host: Spermophilus franklinii

Dist.: Sask

Record: McGee 1980 (Sask)

Alaria taxideae Swanson and Erickson, 1946

Site: intestine

Host: Martes americana (2)

M. a. actuosa (1)

Dist.: Man, NWT

Records: 1. Holmes 1963 (NWT); 2. Poole et al. 1983 (Man)

Alaria spp.

Site: intestine

Hosts: Lynx lynx (2)

Gulo gulo (3)

Martes pennanti (1)

Dist.: Alta, NWT, Ont

Records: 1. Anonymous 1930 (Ont); 2. Zyll

DeJong 1966 (Alta, NWT); 3. Addison and

Boles 1978 (NWT)

Diplostomum mergi Dubois, 1932

Site: intestine

Host: Ondatra zibethicus obscurus

Dist.: Nfld

Record: Rigby and Threlfall 1981

Diplostomum spathaceum (Rudolphi, 1819)

Olsson, 1878

Site: eye

Hosts: Lab. hamster

Lab. rabbit

Dist.: Ont

Record: Lester and Freeman 1976 exp. 
Fibricola cratera (Barker and Noll, 1915) Dubois, 1932

Site: intestine

Host: Mephitis mephitis

Dist.: Que

Record: Webster and Wolfgang 1956

Remarks: Webster and Wolfgang (1956) did not specify the site in which their specimens were found, but presumably it was the intestine.

Fibricola laruei Miller, 1940

Site: small intestine

Host: Procyon lotor

Dist.: Que

Record: Miller 1940

Pharyngostomoides adenocephala Beckerdite,

Miller and Harkema, 1971

Site: intestine

Hosts: Procyon lotor (2)

P. l. hirtus (1)

Dist.: Ont, Sask

Records: 1. Hoberg and McGee 1982 (Sask); 2.

Butterworth and Holmes 1984 (Ont)

Pharyngostomoides procyonis Harkema, 1942

Site: intestine

Host: Procyon lotor

Dist.: Ont

Record: Butterworth and Holmes 1984

\section{FAMILY ECHINOSTOMATIDAE}

Echinochasmus schwartzi Price, 1931

Site: intestine

Host: Ondatra zibethicus

Dist.: Ont

Record: Law and Kennedy 1932

Echinoparyphium contiguum Barker and Bastron, 1915

Sites: duodenum, intestine

Hosts: Ondatra zibethicus $(1,2,3)$

O. z. obscurus (4)

Dist.: BC, Nfld, Ont

Records: 1. Law and Kennedy 1932 (Ont); 2.

Knight 1951 (BC); 3. Sweatman 1952b (Ont);

4. Rigby and Threlfall 1981 (Nfld)

Echinoparyphium recurvatum (Linstow, 1873)

Lühe, 1909

Site: intestine

Host: Ondatra zibethicus obscurus

Dist.: Nfld

Record: Rigby and Threlfall 1981
Echinoparyphium sp.

Site: intestine

Host: Ondatra zibethicus

Dist.: NB

Record: MacKinnon and Burt 1978

Echinostoma callawayensis Barker and Noll, 1915

Sites: duodenum, small intestine

Host: Ondatra zibethicus

Dist.: Ont

Records: Law and Kennedy 1932; Sweatman 1952

Echinostoma revolutum Froelich, 1802

Sites: duodenum, small intestine

Hosts: Castor canadensis (6)

Ondatra zibethicus $(1,2,3,4,5,7,9)$

O. $z$. obscurus (10)

Vulpes vulpes (8)

Dist.: BC, Man, NB, Nfld, NS, Ont, Que

Records: 1. Anonymous 1930 (Ont); 2. Law and

Kennedy 1932 (Ont); 3. Knight 1951 (BC); 4.

Sweatman 1952b (Ont); 5. Gupta 1962b (Que);

6. Smith and Archibald 1967 (NS); 7.

MacKinnon and Burt 1978 (NB); 8. Smith

1978b (NB, NS); 9. McKenzie and Welch 1979

(Man); 10. Rigby and Threlfall 1981 (Nfld)

Echinostoma spp.

Site: intestine

Hosts: Martes pennanti (1)

Ondatra zibethicus (2)

Dist.: Alta, Ont

Records: 1. Anonymous 1930 (Ont); 2. Swales 1933b (Alta)

Euparyphium inerme Fuhrmann, 1904

Site: intestine

Host: Mustela vison

Dist.: Ont

Records: Law and Kennedy 1932; Price 1932

Euparyphium melis Schrank, 1788

Site: duodenum

Host: Mustela vison

Dist.: Ont

Record: Law and Kennedy 1932

\section{FAMILY FASCIOLIDAE}

Fasciola hepatica Linnaeus, 1758

Site: liver

Host: Odocoileus hemionus columbianus

Dist.: BC

Record: Hadwen 1916 
Fascioloides magna (Bassi,1875) Ward, 1917

Syn.: Fasciola magna

Site: liver

Hosts: Alces alces $(10,11$

$\mathrm{BC}, 12,15,18,19,20,21)$

Bison bison $(2,4,5,11)$

Cavia porcellus (4 exp.)

Cervus elaphus $(5,8,9,10,11,13,14,21)$

Cervus spp. (15)

Odocoileus hemionus columbianus

$(1,3,4,7,11)$

O. h. hemionus $(6,11)$

$O$. virginianus $(4,5,10)$

Odocoileus spp. (15)

Oryctolagus cuniculus (4)

Rangifer tarandus caribou (17)

Dist.: Alta, BC, Man, Ont, Que, Sask

Records: 1. Hadwen 1916 (BC); 2. Cameron 1923 (Alta); 3. Hadwen 1932 (BC); 4. Swales 1935 (Alta, BC), 5. 1936 (Que); 6. Cowan 1942 (BC), 7. 1946 (Province?); 8. Kingscote 1947 (Ont); 9. Anonymous 1950 (Ont); 10. Kingscote 1950 (Ont); 11. Cowan 1951 (Alta, BC); 12. Peterson 1955 (Ont); 13. Kingscote 1957 (Ont); 14. Stelfox 1962 (Alta); 15. Fyvie 1966 (Ont); 16. Flook and Stenton 1969 (Alta, BC); 17. Choquette et al. 1971 (Que); 18. Lankester 1972 (Man), 19. 1974 (Man); 20. Samuel et al. 1976 (Alta); 21. Wobeser et al. 1985 (Sask)

\section{FAMILY HETEROPHYIDAE}

Apophallus venustus (Ransom, 1920) Cameron, 1936

Site: intestine

Host: Procyon lotor

Dist.: Can, Ont

Record: Cameron 1936 (Ont), 1937a (Can)

Apophallus sp.

Site: intestine

Host: Mephitis mephitis

Dist.: Que

Record: Webster and Wolfgang 1956

Remarks: Webster and Wolfgang (1956)

believed their species may be Apophallus

venustus.

Cryptocotyle lingua (Creplin, 1825) Lühe, 1899

Site: intestine

Host: Vulpes vulpes

Dist.: NB, NS

Record: Smith $1978 b$
Euryhelmis pyriformis Webster and Wolfgang, 1956

Site: intestine

Host: Mephitis mephitis

Dist.: Que

Record: Webster and Wolfgang 1956

Phagicola longa (Ransom, 1920) Price, 1935

Site: intestine

Host: Vulpes vulpes

Dist.: Ont, Que

Record: Swales 1933b

\section{FAMILY LECITHODENDRIIDAE}

Acanthatrium eptesici Alicata, 1932

Site: digestive tract

Host: Eptesicus fuscus

Dist.: BC

Record: Webster and Casey 1973

Acanthatrium nycteridis Faust, 1919

Site: digestive tract

Host: Lasionycteris noctivagans

Dist.: BC

Record: Webster and Casey 1973

Lecithodendrium anticum Stafford, 1905

Site: intestine

Host: Myotis lucifugus

Dist.: Not specified

Record: Stafford 1905

Lecithodendrium posticum Stafford, 1905

Site: intestine

Host: Myotis lucifugus

Dist.: Not specified

Record: Stafford 1905

Limatulum gastroides Macy, 1935

Site: digestive tract

Host: Lasiurus cinereus

Dist.: BC

Record: Webster and Casey 1973

Prosthodendrium macnabi Macy, 1936

Site: digestive tract

Hosts: Eptesicus fuscus

Dist.: BC

Lasionycteris noctivagans

Record: Webster and Casey 1973

Prosthodendrium naviculum Macy, 1936

Site: digestive tract

Host: Myotis volans longicrus

Dist.: BC

Record: Webster and Casey 1973 
Prosthodendrium silvai (Travassos, 1928)

Site: intestine

Host: Lasiurus cinereus

Dist.: BC

Record: Webster and Casey 1973

Prosthodendrium swansoni Macy, 1936

Site: digestive tract

Host: Myotis lucifugus

Dist.: BC

Record: Webster and Casey 1973

Prosthodendrium spp.

Site: digestive tract

Hosts: Eptesicus fuscus

Lasionycteris noctivagans

Lasiurus cinereus

Dist.: BC

Myotis lucifugus

Record: Webster and Casey 1973

FAMILY MICROPHALLIDAE

Microphallus sp.

Site: intestine

Host: Procyon lotor hirtus

Dist.: Sask

Record: Hoberg and McGee 1982

Remarks: Hoberg and McGee (1982) did not specify the site in which their specimens were found, but presumably it was the intestine.

\section{FAMILY NOTOCOTYLIDAE}

Notocotylus filamentis (Barker, 1915) Harwood, 1939

Syn.: Catatropis filamentis (Barker, 1915)

Sites: small intestine, large intestine

Host: Ondatra zibethicus

Dist.: Man, Ont

Records: Law and Kennedy 1932 (Ont);

Sweatman 1952b (Ont); McKenzie and Welch 1979 (Man)

Notocotylus urbanensis (Cort, 1914) Harrah, 1922

Site: caecum

Host: Ondatra zibethicus

Dist.: BC, NB, Ont

Records: Knight 1951 (BC); Beverley-Burton and Sweeny 1972 (Ont); MacKinnon and Burt 1978 (NB)
Nudacotyle novicia Barker, 1916

Site: intestine

Host: Ondatra zibethicus

Dist.: NB, Ont

Records: Law and Kennedy 1932 (Ont);

MacKinnon and Burt 1978 (NB)

Quinqueserialis quinqueserialis (Barker and Laughlin, 1911) Harwood,1939

Syn.: Notocotylus quinqueserialis Barker and Laughlin, 1911

Site: caecum

Hosts: Microtus pennsylvanicus (4)

Ondatra zibethicus $(1,2,3,5,6,7)$

O. $z$. obscurus (8)

Zapus hudsonius (4)

Dist.: BC, Man, NB, Nfld, Ont, Que

Records: 1. Law and Kennedy 1932 (Ont); 2.

Sweatman 1952b (Ont); 3. Knight 1951 (BC);

4. Schad 1954 (Nfld, Que); 5. Beverley-Burton and Sweeny 1972 (Ont); 6. MacKinnon and

Burt 1978 (NB); 7. McKenzie and Welch 1979

(Man); 8. Rigby and Threlfall 1981 (Nfld)

Quinqueserialis zibethicai Gupta 1962

Site: duodenum

Host: Ondatra zibethicus

Dist.: Que

Record: Gupta 1962a

\section{FAMILY OPISTHORCHIIDAE}

Metorchis conjunctus (Cobbold, 1860) Looss, 1899

Sites: bile ducts, gall bladder, pancreas

Hosts: Canis latrans (2)

Canis lupus $(2,6)$

Martes pennanti (4)

Ondatra zibethicus (1)

Sigmodon hispidus (5)

Vulpes vulpes (3)

Dist.: Alta, Man, NB, NS, Ont, Que, Sask

Records: 1. Sweatman 1952b (Ont); 2. Holmes and Podesta 1968 (Alta); 3. Smith 1978b (NB, NS); 4. Dick and Leonard 1979 (Man); 5.

Watson 1981 exp. (Que); 6. Wobeser et al. 1983 (Sask)

Opisthorchis tonkae Wallace and Penner, 1939

Sites: gall bladder and bile ducts

Host: Ondatra zibethicus

Dist.: NB

Record: MacKinnon and Burt 1978

Remarks: MacKinnon and Burt (1978) did not specify the sites in which their specimens were found, but presumably it was the gall bladder and bile ducts. 
Parametorchis canadensis Price, 1929

Site: gallbladder

Host: Mustela vison

Dist.: Ont

Records: Price 1929; Anonymous 1930, 1931;

Law and Kennedy 1932

\section{FAMILY PARAMPHISTOMIDAE}

Paramphistomum cervi (Zeder, 1790) Fischoeder, 1901

Site: rumen

Host: Alces alces

Dist.: Nfld, Ont, Que, Sask

Records: Peterson 1955 (Ont, Que); Threlfall

1967 (Nfld); Lankester et al. 1979 (Ont);

Kennedy et al. 1985 (Ont, Sask)

Remarks: Kennedy et al. (1985) re-examined

Lankester's et al. (1979) material and

concluded there were two species present:

Paramphistomum cervi and Paramphistomum liorchis.

Paramphistomum liorchis Fischoeder, 1901

Site: rumen

Host: Alces alces

Dist.: Ont

Records: Kennedy et al. 1985

Paramphistomum spp.

Site: rumen

Host: Alces alces

Dist.: Alta

Record: Samuel et al. 1976

Unidentified Paramphistomidae

Paramphistomidae gen. sp.

Site: intestine

Host: Ondatra zibethicus obscurus

Dist.: Nfld

Record: Rigby and Threlfall 1981

Stichorchis subtriquetrus (Rudolphi, 1814)

Fischoeder, 1901

Sites: caecum, small intestine

Hosts: Castor canadensis $(1,2,4,5,6)$

C. c. leucodontus (3)

Dist.: Alta, BC, Nfld, NS, Ont, Que

Records. 1. Duff 1914 (Ont, Que); 2. Law and

Kennedy 1932 (Ont); 3. Cowan 1942 (BC); 4.

Choquette and Pimlott 1956 (Nfld); 5. Smith

and Archibald 1967 (NS); 6. Bush and Samuel

1981 (Alta)
Wardius zibethicus Barker and East, 1915

Sites: caecum, colon

Host: Ondatra zibethicus

Dist.: Man, NB, Ont

Records: Law and Kennedy 1932 (Ont);

MacKinnon and Burt 1978 (NB); McKenzie

and Welch 1979 (Man)

Zygocotyle lunata (Diesing, 1835) Stunkard, 1917

Site: caecum

Host: Alces alces

Dist.: Alta

Records: Samuel et al. 1976; Stock and Barrett 1983

\section{FAMILY PLAGIORCHIIDAE}

Plagiorchis elegans (Rudolphi, 1802)

Site: intestine

Host: Procyon lotor hirtus

Dist.: Sask

Record: Hoberg and McGee 1982

Plagiorchis muris Tanabe, 1922

Site: intestine

Host: Microtus pennsylvanicus

Dist.: Nfld, Que

Record: Schad 1954

Plagiorchis noblei Fedorova, 1969

Site: intestine

Host: Ondatra zibethicus

Dist.: Man

Record: McKenzie and Welch 1979

Plagiorchis proximus Barker, 1915

Sites: small intestine, duodenum

Hosts: Glaucomys sabrinus (8)

Mustela vison $(1,2)$

Ondatra zibethicus $(2,4,5,7)$

O. z. obscurus (9)

Spermophilus franklinii (8)

$S$. richardsonii $(3,8)$

Tamiasciurus hudsonicus (8)

Zapus hudsonius (6)

Dist.: BC, Man, NB, Nfld, Ont, Que, Sask

Records: 1. Anonymous 1930 (Ont); 2. Law and

Kennedy 1932 (Ont); 3. Rausch and Tiner 1948

(Man); 4. Knight 1951 (BC); 5. Sweatman 1952b (Ont); 6. Schad 1954 (Nfld, Que); 7.

MacKinnon and Burt 1978 (NB); 8. McGee

1980 (Sask); 9. Rigby and Threlfall 1981 (Nfld) 
Plagiorchis vespertilionis (Müller, 1784) Braun, 1900

Site: intestine

Hosts: Eptesicus fuscus (2)

Lasionycteris noctivagans (2)

Lasiuris cinereus (2)

Myotis californicus (2)

M. lucifugus $(1,2)$

Dist.: BC

$M$. volans longicrus (2)

Records: 1. Stafford 1905 (not specified); 2.

Webster and Casey 1973 (BC)

Plagiorchis spp.

Site: intestine

Hosts: Clethrionomys gapperi (1)

Eptesicus fuscus (2)

Lasionycteris noctivagans (2)

Dist.: BC, Nfld, Que

Records: 1. Schad 1954 (Nfld, Que); 2. Webster and Casey 1973 (BC)

\section{FAMILY PSILOSTOMIDAE}

Psilostomum ondatrae Price, 1931

Site: liver

Host: Ondatra zibethicus

Dist.: Ont

Record: Law and Kennedy 1932

Stephanoproraoides lawi Price, 1934

Site: small intestine

Host: Castor canadensis

Dist.: NS, Ont

Records: Price 1934 (Ont); Smith and

Archibald 1967 (NS)

\section{FAMILY SCHISTOSOMATIDAE}

Schistosomatium douthitti (Cort, 1914) Price, 1929

Site: hepatic portal vein

Hosts: Erethizon dorsatum (2)

Microtus pennsylvanicus (1)

Ondatra zibethicus (3)

Dist.: Man, NB, Ont

Records: 1. Farley 1962 (Man); 2. Choquette, Broughton et al. 1973 (Ont); 3. MacKinnon and Burt 1978 (NB)

\section{Schistosomatium douthitti ova}

Sites: liver, blood vessels

Hosts: Erethizon dorsatum (1)

Ondatra zibethicus (2)

Dist.: Man, Ont

Records: 1. Choquette, Broughton et al. 1973

(Ont); 2. McKenzie and Welch 1979 (Man)
Schistosomatium douthitti (Cort, 1914) Price, 1929 (immature)

Site: spleen

Host: Ondatra zibethicus

Dist.: Man

Record: McKenzie and Welch 1979

FAMILY TROGLOTREMATIDAE

Nanophyetus salmincola (Chapin, 1926) Chapin, 1927

Site: small intestine

Host: Mustela vison

Dist.: Ont

Record: Anonymous 1930

Paragonimus kellicotti Ward, 1908

Sites: bronchi, bronchioles

Hosts: Canis latrans $(2,3)$

Mephitis mephitis $(2,3)$

Mustela vison $(2,3)$

Vulpes vulpes $(2,3)$

Mink (1)

Dist.: Ont

Record: 1. Fyvie 1966; 2. Presidente and

Ramsden 1975b; 3. Ramsden and Presidente 1975

Paragonimus spp.

Site: intestine

Hosts: Mustela vison (1) Mink (2)

Dist.: Can, Ont

Records: 1. Swales 1933b (Ont); 2. Cameron 1937b (Can)

Digenea gen. spp. metacercariae

Site: liver

Host: Peromyscus maniculatus

Dist.: Que

Record: Schad 1956

\section{CLASS CESTOIDEA SUBCLASS EUCESTODA ORDER CYCLOPHYLLIDEA}

FAMILY ANOPLOCEPHALIDAE

Andrya arctica Rausch, 1952

Site: intestine

Hosts: Dicrostonyx groenlandicus richardsoni

(1)

D. torquatus (3)

Synaptomys borealis (2)

Dist.: Alta, Man, NWT

Records: 1. Rausch 1952a (Man); 2. Lubinsky 1957 (Alta); 3. Webster 1974 (NWT) 
Andrya bairdi Schad, 1954

Site: small intestine

Host: Microtus chrotorrhinus

Dist.: Nfld, Que

Record: Schad 1954

Andrya communis Douthitt, 1915

Site: intestine

Hosts: Clethrionomys gapperi

Microtus pennsylvanicus

Phenacomys intermedius

Dist.: Alta

Record: Lubinsky 1957

Andrya macrocephala Douthitt, 1915

Site: small intestine

Hosts: Clethrionomys gapperi $(1,2)$

Microtus chrotorrhinus $(1,2)$

M. ochrogaster (2)

$M$. pennsylvanicus $(1,2)$

Ondatra zibethicus (3)

Dist.: Alta, NB, Nfld, Que

Records: 1. Schad 1954 (Nfld, Que); 2.

Lubinsky 1957 (Alta); 3. MacKinnon and Burt $1978(\mathrm{NB})$

Andrya neotomae Voge, 1946

Site: intestine

Host: Neotoma cinerea

Dist.: Alta

Record: Lubinsky 1957

Andrya primordialis Douthitt, 1915

Site: small intestine

Host: Tamiasciurus hudsonicus

Dist.: Alta, Sask

Records: Mahrt and Chai 1972 (Alta); McGee 1980 (Sask)

Andrya sciuri Rausch, 1947

Site: intestine

Host: Glaucomys sabrinus

Dist.: Sask

Record: McGee 1980

Andrya sp.

Site: intestine

Host: Clethrionomys gapperi

Dist.: Lab, Que

Record: Schad 1954
Atriotaenia procyonis (Chandler, 1942) Spassky, 1951

Site: intestine

Hosts: Procyon lotor hirtus

Dist.: Sask

Record: Hoberg and McGee 1982

Remarks: Hoberg and McGee (1982) did not specify the site in which their specimens were . found, but presumably it was the intestine.

Avitellina arctica Kolmakov, 1938

Site: intestine

Host: Rangifer tarandus

Dist.: NWT

Record: Gibbs 1959

Avitellina spp.

Site: small intestine

Hosts: Oreamnos americanus (2) Rangifer tarandus (1)

Dist.: Alta, NWT

Records: 1. Gibbs 1960 (NWT?); 2. Samuel, Hall et al. 1978 (Alta)

Cittotaenia pectinata Goeze, 1782

Site: intestine

Host: Lepus americanus struthopus

Dist.: Nfld

Record: Dodds and Mackiewicz 1961

Cittotaenia pectinata americana Stiles and Hassel, 1896

Site: small intestine

Host: Lepus americanus

Dist.: Man

Record: Boughton 1932

Diandrya vancouverensis Mace and Shepard, 1981

Site: small intestine

Host: Marmota vancouverensis

Dist.: BC

Record: Mace and Shepard 1981

Moniezia benedini (Moniez, 1879) Blanchard, 1891

Site: intestine

Hosts: Alces alces (3)

Cervus elaphus (4)

Odocoileus hemionus columbianus

$(2,3)$

O. h. hemionus (3)

Oreamnos americanus $(1,3,5)$

Ovis canadensis (1)

O. c. canadensis (3)

Dist.: Alta, BC

Records: 1. Cowan 1944 (Alta), 2. 1946 (BC), 3. 1951 (Alta, BC); 4. Flook and Stenton 1969 (Alta); 5. Samuel, Hall et al. 1978 (Alta) 
Moniezia expansa (Rudolphi, 1810) Blanchard, 1891

Site: intestine

Hosts: Ovibos moschatus $(2,3,5)$ Ovis canadensis canadensis (4) Rangifer tarandus (1)

Dist.: Alta, NWT, Sask

Records: 1. Erickson and Highby 1942 (Sask); 2. Durrell and Bolton 1957 (NWT); 3. Gibbs and Tener 1958 (NWT); 4. Samuel, Hall et al. 1978 (Alta); 5. Webster and Rowell 1980 (NWT)

Moniezia spp.

Site: small intestine

Hosts: Alces alces (6)

A. a. andersoni (3)

Bison bison (1)

Ovibos moschatus (4)

Rangifer tarandus (2)

R. t. caribou (5)

Dist.: Alta, BC, NWT

Records: 1. Cameron 1923 (Alta); 2. Gibbs 1960 (NWT?); 3. Barrett 1972 (Alta); 4. Samuel and Gray 1974 (NWT, Alta capt.); 5. Low 1976 (BC); 6.Samuel et al. 1976 (Alta) Remarks: This parasite was identified by Low (1976) as probably being Moniezia expansa. This parasite was reported by Cameron (1923) as "...species not identified, but probably planissima."

Oochoristica oklahomensis Peery, 1939

Site: intestine

Host: Mephitis mephitis

Dist.: Que

Record: Choquette 1951

Paranoplocephala infrequens (Douthitt, 1915)

Baer, 1927

Sites: caecum, small intestine

Hosts: Microtus pennsylvanicus $(1,2)$ Synaptomys borealis (1)

Dist.: Alta, Lab, Que

Records: 1. Schad 1954 (Lab, Que); 2. Lubinsky 1957 (Alta)

Paranoplocephala lemmi Rausch, 1952

Site: caecum

Host: Lemmus t. trimucronatus

Dist.: NWT

Record: Rausch $1952 b$
Paranoplocephala variabilis (Douthitt, 1915)

Hansen, 1947

Sites: colon, small intestine

Hosts: Dicrostonyx hudsonius (1) Microtus pennsylvanicus $(1,2)$

Phenacomys intermedius (2) Thomomys talpoides (2)

Dist.: Alta, Nfld, Que

Records: 1. Schad 1954 (Nfld, Que); 2.

Lubinsky 1957 (Alta)

Schizorchis caballeroi Rausch, 1960

Site: small intestine

Hosts: Ochotona collaris

Dist.: Alta, YT

Record: Hobbs 1980

Schizorchis ochotonae Hansen, 1948

Site: small intestine

Host: Ochotona princeps

Dist.: Alta

Record: Lubinsky 1957

Thysanosoma actinioides Diesing, 1834

Sites: duodenum, jejunum

Hosts: Alces alces $(7,9)$

A. a. andersoni (6)

Cervus elaphus $(1,3,5,9)$

Odocoileus hemionus columbianus

$(2,3)$

O.h. hemionus (3 $\mathrm{BC})$

Oreamnos americanus $(1,3,4,8)$

Ovis c. canadensis (3)

Dist.: Alta, BC

Records: 1.Cowan 1944 (Alta), 2. 1946 (BC), 3.

1951 (Alta, BC); 4. Kerr and Holmes 1966

(Alta); 5. Flook and Stenton 1969 (Alta, BC); 6.

Barrett 1972 (Alta); 7. Samuel et al. 1976

(Alta); 8. Samuel, Hall et al. 1978 (Alta, BC);

9. Stock and Barrett 1983 (Alta)

Wyominia tetoni Scott, 1941

Sites: bile duct, hepatic ducts

Host: Ovis dalli

Dist.: YT

Record: Gibbs and Fuller 1959

Anoplocephalidae gen. sp.

Site: intestine

Hosts: Ondatra zibethicus obscurus (2)

Oreamnos americanus (1)

Dist.: Alta, BC, Nfld

Records: 1. Samuel, Hall et al. 1978 (Alta, BC);

2. Rigby and Threlfall 1981 (Nfld) 


\section{FAMILY CATENOTAENIIDAE}

Catenotaenia dendritica (Goeze, 1782) Janicki, 1904

Site: small intestine

Host: Clethrionomys gapperi

Dist.: Alta, Nfld, Que

Records: Schad 1954 (Nfld, Que); Lubinsky

1957 (Alta)

\section{FAMILY DILEPIDIDAE}

Choanotaenia nebraskensis Hansen, 1950

Site: small intestine

Host: Mus musculus

Dist.: Alta

Record: Lubinsky 1957

Choanotaenia peromysci (Erickson, 1938)

Site: small intestine

Host: Peromyscus maniculatus

Dist.: Alta

Record: Lubinsky 1957

Choanotaenia spermophili (McLeod, 1933)

Syn: Prochoanotaenia spermophili McLeod, 1933

Site: intestine

Hosts: Spermophilus franklinii $(1,2)$

S. richardsonii $(1,2)$

Dist.: Man, Sask

Records: 1. McLeod 1933 (Man); 2. McGee 1980

(Sask)

Choanotaenia sp.

Site: small intestine

Host: Zapus hudsonius

Dist.: Alta

Record: Lubinsky 1957

Dipylidium caninum (Linnaeus, 1758) Leuckart, 1863

Site: intestine

Hosts: Vulpes vulpes

Dist.: Que

Record: Swales 1933b

Paruterina candelabraria (Goeze, 1782)

(plerocercoids)

Site: liver

Hosts: Microtus pennsylvanicus (1)

Peromyscus maniculatus (1)

Tamiasciurus hudsonicus $(2,3)$

Dist.: Alta, Man, Sask

Records: 1. Baron 1971 (Man); 2. Mahrt and

Chai 1972 (Alta); 3. McGee 1980 (Sask)
Paruterina rauschi Freeman, 1957

(metacestode)

Site: liver

Hosts: Clethrionomys gapperi

Peromyscus maniculatus

Dist.: Ont

Record: Freeman and Wright 1960

Remarks: Freeman and Wright (1960) did not specify the site in which their metacestodes were found, but presumably it was the liver.

\section{FAMILY HYMENOLEPIDIDAE}

Hymenolepis bennetti Freeman, 1960

Site: small intestine

Hosts: Napaeozapus i. insignis

Dist.: Ont

Peromyscus maniculatus gracilis

Record: Freeman 1960

Hymenolepis citelli (McLeod, 1933)

Syn.: Weinlandia citelli (McLeod, 1933)

Site: intestine, stomach

Hosts: Spermophilus franklinii

S. richardsonii

S. tridecemlineatus

Dist.: Man, Sask

Records: McLeod 1933 (Man); McGee 1980

(Sask)

Hymenolepis diminuta Kudolphi, 1819

Site: small intestine

Hosts: Rattus norvegicus (1)

Spermophilus richardsonii (2)

Dist.: Alta, Que

Records: 1. Firlotte 1948 (Que); 2. Lubinsky 1957 (Alta)

Hymenolepis evaginata (Barker and Andrews, 1915)

Site: intestine

Hosts: Ondatra zibethicus $(1,2,3,4,5,6)$

O. . obscurus (7)

Dist.: Alta, BC, NB, Nfld, Ont

Records: 1. Anonymous 1930 (Ont); 2. Law and Kennedy 1932 (Ont); 3. Knight 1951 (BC); 4.

Sweatman 1952b (Ont); 5. Lubinsky 1957 (Alta);

6. MacKinnon and Burt 1978 (NB); 7. Rigby and Threlfall 1981 (Nfld)

Hymenolepis gertschi Macy, 1947

Site: digestive tract

Host: Eptesicus fuscus

Dist.: BC

Record: Webster and Casey 1973 
Hymenolepis horrida von Linstow, 1901

Site: small intestine

Hosts: Clethrionomys gapperi $(1,2,3)$

Microtus chrotorrhinus (1)

$M$. pennsylvanicus (3)

Tamiasciurus hudsonicus (4)

Dist.: Alta, Nfld, Que

Records: 1. Schiller 1952 (Que); 2. Schad 1954

(Nfld, Que); 3. Lubinsky 1957 (Alta); 4. Mahrt and Chai 1972 (Alta)

Hymenolepis johnsoni Schiller, 1952

Site: intestine

Host: Microtus pennsylvanicus drummondi

Dist.: NWT

Record: Rausch 1952

Remarks: Rausch (1952) did not specify the site in which this specimen was found, but presumably it was the intestine.

Hymenolepis murinae (Meggitt, 1927)

Site: intestine

Host: Rattus norvegicus

Dist.: Que

Record: Firlotte 1948

Hymenolepis octocoronata (von Linstow)

Fuhrmann, 1924

Site: small intestine

Host: Ondatra zibethicus

Dist.: BC

Record: Knight 1951

Hymenolepis spp.

Site: small intestine

Hosts: Eutamias minimus (4)

Myotis lucifugus (2)

Ondatra zibethicus $(1,3)$

Dist.: Alta, BC, Man, Sask

Records: 1. Swales 1933b (Alta); 2. Webster and Casey 1973 (BC); 3. McKenzie and Welch 1979 (Man); 4. McGee 1980 (Sask)

Unidentified Hymenolepididae

Hymenolepididae gen. sp.

Site: not specified

Hosts: Rattus norvegicus

Dist.: Nfld

Record: Smith and Threlfall 1973
FAMILY MESOCESTOIDIDAE

Mesocestoides kirbyi Chandler, 1944

Site: intestine

Hosts: Canis latrans (1)

C. lupus (2)

Dist.: Alta, YT

Records: 1. Holmes and Podesta 1968 (Alta); 2.

Choquette, Gibson et al. 1973 (YT)

Mesocestoides latus Mueller, 1928

Site: intestine

Host: Mephitis mephitis

Dist.: Que

Records: Choquette 1951; Webster and

Wolfgang 1956

Mesocestoides spp.

Site: intestine

Hosts: Canis latrans (3)

Martes americana (2)

Mustela vison (2)

Vulpes vulpes (4)

Zapus hudsonius (1)

Dist.: Nfld, Ont, Que

Records: 1. Schad 1954 (Nfld, Que); 2.

Freeman 1956 (Ont); 3. Freeman et al. 1961

(Ont); 4. Freeman 1962 (Ont)

\section{FAMILY TAENIIDAE}

Cladotaenia circi Yamaguti, 1935

(cysticercoid)

Site: liver

Host: Spermophilus tridecemlineatus

Dist.: Sask

Record: McGee 1980

Cladotaenia globifera (Batsch, 1786)

(metacestode)

Site: liver

Hosts: Clethrionomys gapperi (1)

Glaucomys sabrinus (3)

Microtus pennsylvanicus (2)

Peromyscus maniculatus (1)

Dist.: Man, Ont, Sask

Records: 1. Freeman and Wright 1960 (Ont); 2.

Baron 1971 (Man); 3. McGee 1980 (Sask)

Cladotaenia mirsoevi Skrjabin and Popoff, 1924

Site: ileum

Hosts: Clethrionomys gapperi

Clethrionomys sp.

Dist.: Alta

Record: Lubinsky 1957 
Cladotaenia spp. cysticercus

Site: liver

Hosts: Ondatra zibethicus (1)

Tamiasciurus hudsonicus (2)

Dist.: Alta, BC

Records: 1. Knight 1951 (BC); 2. Mahrt and

Chai 1972 (Alta)

Cysticercus fasciolaris Batsch, 1786

Site: liver cysts

Hosts: Ondatra zibethicus $(2,3)$

Rattus norvegicus (1)

Dist.: BC, Ont, Que

Records: 1. Firlotte 1948 (Que); 2. Knight 1951

(BC); 3. Sweatman 1952b (Ont)

Cysticercus ovis Cobbold, 1869

Site: muscle cysts

Host: Odocoileus hemionus columbianus

Dist.: BC

Record: Cowan 1946

Cysticercus pisiformis Block, 1780

Site: intestine cysts

Hosts: Cottontail rabbit (2)

Lab. rabbit (3)

Rabbit (1)

Dist.: Ont

Varying hare (2)

Records: 1. Law 1933b; 2. Fyvie 1966; 3.

Barker 1970 exp.

Cysticercus talpae Rudolphi, 1819

Site: liver cysts

Hosts: Ondatra zibethicus

Dist.: Ont

Record: Skinker 1935

Cysticercus tarandi Moniez, 1789

Site: muscle cysts

Hosts: Rangifer tarandus (1)

Moose (2)

Dist.: NWT, Ont

Records: 1. Gibbs 1960 (NWT?); 2. Fyvie 1966

(Ont)
Cysticercus tenuicollis Pallas, 1766

Site: liver cysts

Hosts: Alces alces $(1,2,6,10)$

Cervus elaphus $(5,6,10)$

Odocoileus hemionus $(5,9)$

$O$. h. columbianus $(5,6)$

O. h. hemionus (6)

O. virgianus ochrourus (6)

Oreamnos americanus $(4,6)$

Ovibos moschatus (7)

Ovis canadensis (4)

O. c. canadensis (6)

Rangifer tarandus $(6,9)$

R.t. terraenovae (1)

Deer (10)

Dist.: Alta, BC, Nfld, NWT, Ont

Records: 1. Dikmans 1932 (Nfld); 2. Hadwen 1932 (Alta); 3. Gregson 1937 (BC); 4. Cowan 1944 (Alta), 5. 1946 (BC), 6. 1951 (Alta, BC); 7. Gibbs and Tener 1958 (NWT); 8. Ritcey and Edwards 1958 (BC); 9. Gibbs 1960 (NWT?); 10. Fyvie 1966 (Ont); 11. Threlfall 1969 (Nfld)

Cysticercus spp. cyst

Sites: cysts in muscle and body cavity

Hosts: Alces alces $(2,3,4,5)$

Odocoileus hemionus columbianus (1)

Rangifer tarandus (1)

Dist.: Alta, BC, NS, Ont, Sask, YT

Records: 1. Cowan 1951 (Alta, BC); 2. Peterson 1955 (Ont); 3. Benson 1958 (NS); 4. Ritcey and Edwards 1958 (BC); 5. Sweatman and Henshall 1962 (Ont, Sask, YT)

Echinococcus granulosus (Batsch, 1786) Rudolphi, 1801

Site: intestine

Hosts: Canis latrans $(1,3,4,5,6,8)$

C. lupus $(1,2,3,4,5,6,7,8,9)$

Martes pennanti (3)

Dist.: Alta, Man, NWT, Ont, Que, YT

Records: 1. Cowan 1948 (Alta); 2. DeVos and

Allin 1949 (Ont); 3. Sweatman 1952a (Ont); 4.

Freeman et al. 1961 (Ont); 5. Holmes 1961

(Alta); 6. Holmes and Podesta 1968 (Alta); 7.

Choquette, Gibson et al. 1973 (NWT, YT); 8.

Samuel, Ramalingam and Carbyn 1978 (Man);

9. McNeill et al. 1984 (Que) 
Echinococcus granulosus (Batsch, 1786) Rudolphi, 1801

(hydatid cyst)

Sites: cysts in lungs and liver

Hosts: Alces alces $(1,5,7,8,10,11,13,15,16,19$, $20,21,22,24,25,26,27,28,29)$

A. a. andersoni (23)

Bison bison (20)

Cervus elaphus $(2,4,6,7,19,20,23)$

Odocoileus hemionus columbianus

$(3,7)$

O. h. hemionus $(4,7)$

O. virginianus $(8,10,18,20)$

Ovibos moschatus (14)

Rangifer tarandus $(10,12,15$

NWT, 17,20)

R.t. groenlandicus (9)

Rangifer sp. (20)

Deer (21)

Dist.: Alta, BC, Man, NS, NWT, Ont, Que, Sask, YT

Records: 1. Hadwen 1932 (Man); 2. Cowan 1944 (Alta), 3. 1946 (BC), 4. 1948 (Alta); 5. DeVos and Allin 1949 (Ont); 6. Green 1949 (Alta); 7. Cowan 1951 (Alta, BC); 8. Sweatman 1952a (Ont); 9. Banfield 1954 (Man); 10. Harper et al. 1955 (Sask, NWT); 11. Peterson 1955 (Ont); 12. Choquette et al. 1957 (NWT); 13. Benson 1958 (NS); 14. Gibbs and Tener 1958 (NWT); 15. Lubinsky 1958 (Alta, NWT); 16. Ritcey and Edwards 1958 (BC); 17. Gibbs 1960 (NWT?); 18. Anderson 1962b (Ont); 19. Stelfox 1962 (Alta); 20. Sweatman and Williams 1963 (Alta, NWT, Ont, Sask, YT); 21. Fyvie 1966 (Ont); 22. Flook and Stenton 1969 (Alta); 23. Barrett 1972 (Alta); 24. Samuel 1972b (Alta); 25. Samuel et al. 1976 (Alta); 26. Addison et al. 1979 (Ont); 27. Crichton 1979 (Man); 28. Rau and Caron 1979 (Que); 29. Crichton 1980 (Man)

Remarks: Benson (1958) assumed the cysts in the liver were hydatid cysts.

Echinococcus granulosus (Batsch, 1786) Rudolphi, 1801

(Lebanon strain)

Site: liver cysts

Hosts: Mus musculus lab.

Sigmodon hispidus

Dist.: Que

Record: Webster and Cameron 1961 exp.
Echinococcus granulosus canadensis

Site: liver cysts

Hosts: Lab. mice

Dist.: Que

A.K.R. mice

Record: Webster and Cameron 1961 exp.

Echinococcus multilocularis Leuckart, 1863

Site: intestine

Hosts: Alopex lagopus $(1,5)$

Canis latrans (4)

Vulpes vulpes $(2,3,4)$

Dist.: Man, NWT, Sask

Records: 1. Choquette et al. 1962 (NWT); 2.

Hnatiuk 1969 (Sask); 3. Baron 1970 (Man); 4.

Samuel, Ramalingam et al. 1978 (Man); 5.

Eaton and Secord 1979 (NWT)

Echinococcus multilocularis Leuckar, 1863

(hydatid cyst)

Sites: liver, peritoneum, subcutaneous

Hosts: Meriones unguiculatus (7)

Microtus pennsylvanicus (1)

Peromyscus maniculatus $(2,3,4,8)$

Sigmodon hispidus $(5,6,7)$

A/J lab. mice (7)

Dist.: Alta, Man, Que, Sask

Records: 1. Hnatiuk 1966 (Sask); 2. Lee 1969

(Man); 3. Leiby et al. 1969 (Man); 4. Holmes et al. 1971 (Alta); 5. Rau and Tanner 1972 exp.

(Que); 6. Rau and Tanner 1973 exp. (Que); 7.

Baron et al. 1974 exp. (Que); 8. Chalmers and

Barrett 1974 (Alta)

Echinococcus multilocularis sibiricensis Vogel, 1955

Syn.: Echinococcus sibiricensis Rausch and Schiller, 1954

Site: liver cysts

Hosts: Meriones unguiculatus

Mus musculus lab.

Ondatra zibethicus

Sigmodon hispidus

Lab. mice

Lab. rats

Dist.: Que

Record: Webster and Cameron 1961 exp.

Echinococcus sp.

Site: liver

Host: Bison bison

Dist.: Alta

Records: Cameron 1923 
Multiceps sp.

Site: intestine

Host: Lynx canadensis

Dist.: Alta, NWT

Record: Zyll de Jong 1966

Multiceps sp. cyst

Site: skeletal muscle cysts

Host: Lepus americanus struthopus

Dist.: Nfld

Record: Dodds and Mackiewicz 1961

Remarks: Dodds and Mackiewicz (1961) did not specify the site in which the cysts were found, but presumably they were in skeletal muscle.

Taenia crassiceps (Zeder, 1800) Rudolphi, 1810

Site: small intestine

Hosts: Alopex lagopus (6)

Canis lupus $(1,4)$

Vulpes vulpes $(2,3,4,5)$

Vulpes sp. (1)

Coyote x Dog (1)

Dist.: Man, NB, NS, NWT, Ont

Records: 1. Freeman et al. 1961 (Ont); 2.

Freeman 1962 (Ont); 3. Baron 1970 (Man); 4.

Samuel, Ramalingam et al. 1978 (Man); 5.

Smith 1978b (NB, NS); 6. Eaton and Secord 1979 (NWT)

Taenia crassiceps (Zeder, 1800) Rudolphi, 1810

(Cysticercus)

Sites: Subcutaneous tissue, thoracic and abdominal cavities

Hosts: Dicrostonyx groenlandicus richardsoni

(1)

D. torquatus (4)

Marmota monax (2)

Microtus pennsylvanicus (2)

M. p. drummondii (3)

Ondatra zibethicus (2)

Peromyscus maniculatus (2 exp.)

Sciurus carolinensis (2 exp.)

Tamias striatus (2)

Tamiasciurus hudsonicus (2 exp.)

Cotton rat (3 exp.)

Gerbil (3 exp.)

Lab. mouse (3 exp.)

White mice ( 2 exp.)

Dist.: Man, NWT, Ont

Records: 1. Rausch 1952b (Man); 2. Freeman

1962 (Ont); 3. Lubinsky and Baron 1970

(Man); 4. Webster 1974 (NWT)
Taenia hydatigena Pallas, 1766

Site: intestine

Hosts: Canis latrans $(4,5,8)$
C. lupus $(2,3,4,5,6,8,9,10)$
Felis concolor (2)
F. c. vancouverensis (1)
Ursus americanus $(7,9)$

Dist.: Alta, BC, Man, NWT, Ont, Que, YT

Records: 1. Cowan 1942 (BC), 2. 1944 (Alta); 3.

Sweatman and Plummer 1957 exp. (Ont); 4.

Freeman et al. 1961 (Ont); 5. Holmes and

Podesta 1968 (Alta); 6. Choquette, Gibson et

al. 1973 (NWT, YT); 7. Frechette and Rau 1977 (Que); 8. Samuel, Ramalingam and Carbyn 1978 (Man); 9. Dies 1979 (Alta); 10. McNeill et al. 1984 (Que)

Taenia hydatigena Pallas, 1766

(Cysticercus)

Site: liver

Hosts: Alces alces $(3,5,9,11,15)$

A. a. andersoni (7)

Cervus elaphus $(5,15)$

Odocoileus virginianus (4)

Odocoileus sp. (3)

Oreamnos americanus $(6,10)$

Ovibos moschatus $(8,14)$

Ovis canadensis canadensis (10)

Rangifer tarandus $(2,3)$

R.t. groenlandicus (1)

Deer (5)

Moose $(12,13)$

Dist.: Alta, BC, Man, NWT, Ont, Que, Sask

Records: 1. Banfield 1954 (not specified); 2.

Choquette et al. 1957 (NWT); 3. Sweatman and Plummer 1957 (Alta, BC, NWT, Ont, Que, Sask); 4. Anderson 1962b (Ont); 5. Stelfox 1962 (Alta); 6. Kerr and Holmes 1966 (Alta); 7 Barrett 1972 (Alta); 8. Samuel 1972b (Alta); 9. Samuel et al. 1976 (Alta); 10. Samuel, Hall et al. 1978 (Alta); 11. Addison et al. 1979 (Ont); 12. Crichton 1979 (Man), 13. 1980 (Man); 14. Webster and Rowell 1980 (NWT); 15. Stock and Barrett 1983 (Alta)

Taenia krabbei Moniez, 1879

Site: intestine

Hosts: Canis latrans (2)

C. lupus $(1,2,4)$

Ursus americanus $(6,5,7)$

$U . \operatorname{arctos}(3)$

Dist.: Alta, NWT, Ont, Que, YT

Records: 1. Freeman et al. 1961 (Ont); 2.

Holmes and Podesta 1968 (Alta); 3. Choquette et al. 1969 (NWT, YT?); 4. Choquette et al. 1973 (NWT, YT); 5. Frechette and Rau 1977 (Que); 6. Addison et al. 1978 (Ont); 7. Dies 1979 (Alta) 
Taenia krabbei Moniez, 1879

(cysticercus)

Sites: cardiac muscle, skeletal muscle

Hosts: Alces alces $(4,6,7,9,10,11,12)$

A. a. andersoni (8)

Rangifer tarandus $(1,2,5)$

R. t. groenlandicus $(3,7)$

Moose (13)

Dist.: Alta, Man, NWT, Ont, Que, Sask, YT

Records: 1. Erickson and Highby 1942 (Sask);

2. Cowan 1944 (Alta); 3. Banfield 1954 (not

specified); 4. Peterson 1955 (Que); 5.

Choquette et al. 1957 (NWT); 6. Stelfox 1962

(Alta); 7. Sweatman and Henshall 1962 (NWT,

Ont, Sask, YT); 8. Barrett 1972 (Alta); 9.

Samuel 1972a (Alta), 10. 1972b (Alta); 11.

Samuel et al. 1976 (Alta); 12. Addison et al.

1979 (Ont); 13. Crichton 1979 (Man)

Taenia laticollis Rudolphi, 1819

Site: intestine

Hosts: Canis latrans (1)

C. lupus (1)

Lynx canadensis $(2,3,4)$

L. rufus (2)

Dist.: Alta, BC, Nfld, NWT, Ont

Records: 1. Freeman et al. 1961 (Ont); 2.

Adams 1964 (BC); 3. Zyll de Jong 1966 (Alta,

NWT); 4. Threlfall 1969 (Nfld)

Taenia macrocystis (Diesing, 1850) Lühe, 1910

Site: small intestine

Hosts: Lynx canadensis

L. rufus

Dist.: NB, Nfld, NS

Record: Bursey and Burt 1970

Taenia macrocystis (Diesing, 1850) Lühe, 1910 (metacestode)

Site: unspecified

Host: Lepus americanus (1)

Dist.: NB, NS

Record: Bursey and Burt 1970

Taenia martis (Zeder, 1803) Wahl, 1967

Site: digestive tract

Hosts: Gulo gulo (3)

Martes americana (1)

M. a. actuosa (2)

Dist.: NWT, Ont

Records: 1. Freeman 1956 (Ont); 2. Holmes 1963 (NWT); 3. Addison and Boles 1978

(NWT)
Taenia multiceps Leske, 1780

Syn: $\quad$ Multiceps multiceps (Leske, 1780)

Site: intestine

Hall, 1919

Hosts: Canis latrans

Dist.: Alta

C. lupus

Record: Holmes and Podesta 1968

Taenia mustelae Gmelin, 1790

Syn.: Taenia tenuicollis Rudolphi, 1819

Site: intestine

Hosts: Martes americana (5)

M. a. actuosa (3)

Mustela erminea $(2,4)$

M. putorius (2 exp.)

$M$. vison $(1,2$ exp. 4$)$

Dist.: Man, Nfld, NWT, Ont

Records: 1. Skinker 1935 (Ont); 2. Freeman 1956 (Ont); 3. Holmes 1963 (NWT); 4.

Jennings et al. 1982 (Nfld); 5. Poole et al.1983

(Man)

Remarks: Verster (1969) noted that $T$.

tenuicollis is a synonym of $T$. mustelae.

Taenia mustelae Gmelin, 1790

(metacestode)

Syn.: Taenia tenuicollis Rudolphi, 1819

Sites: liver, lungs, kidneys, mesenteries

Hosts: Clethrionomys gapperi $(1,3,5,6)$

Eutamias minimus $(3,7)$

Marmota monax (3)

Microtus ochrogaster (5)

$M$. pennsylvanicus $(1,3,5)$

Mus musculus (3 exp.)

Mustela putorius (3 exp.)

M. vison (3 exp.)

Ondatra zibethicus $(3,3$ exp.,6)

Peromyscus leucopus (4)

$P$. maniculatus $(1,2,3,6)$

Spermophilus franklinii (7)

S. tridecemlineatus (7)

Synaptomys borealis (1)

Tamias striatus (3)

Tamiasciurus hudsonicus (3 exp.,7)

Thomomys talpoides (5)

Zapus hudsonius (3)

Dist.: Alta, NB, Nfld, NWT, Ont, Que, Sask Records: 1. Schad 1954 (Nfld, Que), 2. 1956

(Que); 3. Freeman 1956 (Ont); 4. Gibbs 1957

(NWT); 5. Lubinsky 1957 (Alta); 6. Freeman and Wright 1960 (Ont); 7. MacKinnon and Burt 1978 (NB); 8. Mahrt and Chai 1972 (Alta); 9. McGee 1980 (Sask)

Remarks: Verster (1969) noted that $T$. tenuicollis is a synonym of $T$. mustelae. 
Taenia omissa Lühe, 1910

Site: digestive tract

Hosts: Canis lupus

Felis concolor

Dist.: Alta, BC

Record: Holmes and Podesta 1968

Taenia ovis (Cobbold, 1869) Ransom, 1913

(cysticercus)

Site: muscle

Host: Rangifer tarandus groenlandicus

Dist.: NWT

Record: Banfield 1954

Taenia ovis / T. krabbei Moniez, 1879

(cysticercus)

Site: muscle

Hosts: Alces alces

Odocoileus hemionus columbianus Rangifer tarandus

Dist.: Alta, BC

Record: Cowan 1951

Taenia pisiformis (Bloch, 1780) Gmelin, 1790

Site: intestine

Hosts: Canis latrans $(2,5,7)$

C. lupus $(1,2,5,6)$

Lynx canadensis (4)

Vulpes vulpes (3)

Coyote x Dog (2)

Dist.: Alta, Man, NWT, Ont

Records: 1. Law and Kennedy 1932 (Ont); 2.

Freeman et al. 1961 (Ont); 3. Freeman 1962

(Ont); 4. Zyll de Jong 1966 (Alta, NWT); 5.

Holmes and Podesta 1968 (Alta); 6. Choquette

et al. 1973 (NWT); 7. Samuel, Ramalingam et al. 1978 (Man)

Taenia pisiformis (Bloch, 1780) Gmelin, 1790 (cysticerci)

Sites: abdominal cavity, liver, skeletal muscle

Hosts: Lepus americanus $(1,2,4,5)$

L. a. struthopus (3)

Dist.: Alta, Man, Nfld

Records: 1. Boughton 1932 (Man); 2. Lubinsky 1957 (Alta); 3. Dodds and Mackiewicz 1961

(Nfld); 4. Smith and Threlfall 1973 (Nfld); 5.

Keith et al. 1985 (Alta)

Taenia polyacantha Leukart, 1856

Site: intestine

Host: Alopex lagopus

Dist.: NWT

Record: Webster 1974
Taenia rileyi Loewen, 1929

Site: intestine

Hosts: Lynx canadensis $(1,2)$

L. rufus (1)

Dist.: Alta, BC, NWT

Records: 1. Adams 1964 (BC); 2. Zyll de Jong

1966 (Alta, NWT)

Remarks: The records of Adams (1964)

involved a tentative parasite identification.

The parasite's distinction from Taenia omissa was uncertain.

Taenia rileyi Loewen, 1929

(cyticerci)

Site: unspecified

Host: Tamiasciurus hudsonicus

Dist.: Alta

Record: Mahrt and Chai 1972

Taenia serialis (Gervais, 1847) Baillet, 1863

Site: intestine

Host: Canis lupus

Dist.: NWT, YT

Record: Choquette et al. 1973

Taenia serialis (Gervais, 1847) Baillet, 1863

(coenurus)

Syn.: $\quad$ Multiceps serialis (Gervais, 1847)

Stiles and Stevenson, 1905

Site: body cavity, connective tissue, liver

Hosts: Lepus americanus $(1,5)$

L. a. pallidus (2)

Varying hare $(3,4)$

Dist.: Alta, BC, Man, Nfld, Ont

Records: 1. Boughton 1932 (Man); 2. Cowan

1942 (BC); 3. Freeman et al. 1961 (Ont); 4.

Fyvie 1966 (Ont); 5. Smith and Threlfall 1973

(Nfld); 6. Keith et al. 1985 (Alta)

Taenia sibirica Dubnitzky, 1952

Site: intestine

Host: Martes pennanti

Dist.: Man

Record: Dick and Leonard 1979

Taenia taeniaeformis (Batsch, 1786) Wolffügel, 1911

Site: digestive tract

Hosts: Canis lupus (2)

Felis concolor vancouverensis (1)

Lynx rufus fasciatus (1)

Dist.: Alta, BC

Records: 1. Cowan 1942 (BC); 2. Holmes and

Podesta 1968 (Alta) 
Taenia taeniaeformis (Batsch, 1786) Wolffügel, 1911

(cysticercus)

Site: liver

Hosts: Lepus americanus struthopus (5)

Mus musculus (4)

Ondatra zibethicus $(1,2,7)$

O. z. osoyoosensis (3)

Rattus norvegicus (6)

Dist.: Alta, BC, Man, Nfld, Ont

Records: 1. Anonymous 1930 (Ont); 2. Law and

Kennedy 1932 (Ont); 3. Knight 1951 (BC); 4.

Lubinsky 1957 (Alta); 5. Dodds and

Mackiewicz 1961 (Nfld); 6. Smith and

Threlfall 1973 (Nfld); 7. McKenzie and Welch

1979 (Man)

Taenia taxidiensis Skinker, 1935

(cysticerus)

Site: musculature of rear legs

Host: Spermophilus franklinii

Dist.: Sask

Record: McGee 1980

Taenia twitchelli Schwartz, 1924

Site: digestive tract

Hosts: Canis latrans (1)

$$
\text { Gulo gulo (2) }
$$

Dist.: Alta, NWT

Records: 1. Holmes and Podesta 1968 (Alta); 2.

Addison and Boles 1978 (NWT)

Unidentified Taenia spp.

Sites: digestive tract, small intestine

Hosts: Canis latrans (7)

Canis lupus $(2,6,7,9)$

Lynx canadensis (5)

Martes americana $(4,8)$

Mustela vison (1)

Vulpes vulpes $(3,7)$

Dist.: Alta, Man, NWT, Ont, Que, YT

Records: 1. Law and Kennedy 1932 (Ont); 2.

DeVos and Allin 1949 (Ont); 3. Freeman 1962

(Ont); 4. Holmes 1963 (NWT); 5. Zyll de Jong

1966 (Alta, NWT); 6. Choquette et al. 1973

(NWT, YT); 7. Samuel, Ramalingam and

Carbyn 1978 (Man); 8. Poole et al. 1983 (Man);

9. McNeill et al. 1984 (Que)
Taenia spp. metacestode

Sites: myocardium, peritoneal cavity

Hosts: Alces alces (2)

Microtus pennsylvanicus drummondi

(3)

Ondatra zibethicus (1)

Dist.: Man, Ont

Records: 1. Anonymous 1930 (Ont); 2. DeVos and Allin 1949 (Ont); 3. Rausch and Tiner 1949 (Man)

Unidentified Taeniid ova

Site: intestine

Host: Ursus americanus

Dist.: Que

Record: Frechette and Rau 1977

Remarks: Frechette and Rau (1977) believed their material was probably $T$. krabbei and/or T. hydatigena.

\section{ORDER PSEUDOPHYLLIDEA}

\section{FAMILY DIPHYLLOBOTHRIIDAE}

Diphyllobothrium cordatum Leuckart, 1863

Site: intestine

Host: Vulpes vulpes

Dist.: Ont

Record: Law and Kennedy 1932 (Ont)

Diphyllobothrium latum Linnaeus, 1758

Site: intestine

Host: Vulpes vulpes

Dist.: Ont

Record: Law and Kennedy 1932

Diphyllobothrium ursi Rausch, 1954

Site: intestine

Hosts: Ursus americanus $(2,3)$

$U . \operatorname{arctos}(1)$

Dist.: BC, Que, YT

Records: 1. Choquette et al. 1969 (BC, YT); 2.

Frechette and Rau 1977 (Que); 3. Juniper 1978 (Que)

Diphyllobothrium ursi Rausch, 1954

(eggs)

Site: intestine

Host: Ursus americanus

Dist.: Que

Record: Frechette and Rau 1978 
Diphyllobothrium spp.

Site: digestive tract

Hosts: Canis latrans (3)

C. lupus $(1,3,4)$

Gulo gulo (5)

Vulpes vulpes (2)

Dist.: Alta, NWT, Ont

Records: 1. DeVos and Allin 1949 (Ont); 2.

Freeman 1962 (Ont); 3. Holmes and Podesta

1968 (Alta); 4. Choquette et al. 1973 (NWT); 5.

Addison and Boles 1978 (NWT)

\section{Diphyllobothrium sp. eggs}

Site: intestine

Host: Ursus americanus

Dist.: Que

Record: Frechette and Rau 1977

Schistocephalus solidus (Müller, 1776) Steenstrup, 1857

(plerocercoid)

Site: gastro-intestinal tract

Host: Lutra canadensis

Dist.: Nfld

Record: Smith and Threlfall 1973

Remarks: The plerocercoid was probably acquired by eating infected fish, and is not considered a true parasite of Lutra canadensis.

\section{Unidentified Cestoda}

Cestoda gen. spp.

Site: digestive tract, muscle

Hosts: Alces alces (1)

Clethrionomys gapperi (2)

Lasiurus cinereus (3)

Dist.: BC, Nfld, Que

Records: 1. Cowan 1942 (BC); 2. Schad 1954 (Nfld, Que); 3. Webster and Casey 1973 (BC)

PHYLUM

NEMATHELMINTHES

CLASS NEMATODA

SUBCLASS ADENOPHOREA

ORDER ENOPLIDA

SUPERFAMILY

DIOCTOPHYMATOIDEA

FAMILY DIOCTOPHYMATIDAE
Dioctophyma renale (Goeze, 1782) Collet-Meygret, 1802

Site: kidney

Hosts: Canis latrans (5)

C. lupus (9)

Mustela vison $(1,2,3,6,7,8)$

Mink (4)

Dist.: Alta, Man, Ont, Que

Records: 1. Anonymous 1930 (Ont); 2. Law and

Kennedy 1932 (Ont); 3.Swales 1933b (Que); 4.

Fyvie 1966 (Ont); 5. Holmes and Podesta 1968

(Alta); 6. Crichton and Urban 1970 (Man); 7.

Mace and Anderson 1975 (Ont); 8. Mace 1976

(Ont); 9. McNeill et al. 1984 (Que)

Eustrongylides sp.larva

Site: muscle

Host: Ondatra zibethicus

Dist.: Ont

Record: Gibson and McKiel 1972

\section{SUPERFAMILY TRICHINELLOIDEA}

FAMILY TRICHINELLIDAE

Trichinella spiralis (Owen, 1835) Railliet, 1894

Site: small intestine

Hosts: Martes pennanti (4)

Mesocricetus auratus (3)

Mus musculus lab. $(2,3,5)$

Rattus sp. (1)

Ursus maritimus (3)

Lab. rat (3)

Dist.: Man, NB, NS, PEI, Que

Records: 1. Frank 1951 (NB, NS, PEI); 2

Shanta and Meerovitch 1967a (Que); 3. Dick and Belosevic 1978 (Man); 4. Dick and

Leonard 1979 (Man); 5. Dick and Chadee 1983

(Man)

Trichinella spiralis (Owen, 1835) Railliet, 1894

(cyst/larva)

Sites: diaphragm, tongue, skeletal muscle

Hosts: Alopex lagopus (14)

Canis latrans $(9,11)$

C. lupus $(8,9,13)$

Felis concolor (11)

Gulo gulo $(11,13)$

Lynx canadensis (11)

L. rufus $(9,11)$

Martes americana $(14,15)$

Mephitis mephitis (11) 
Mus musculus lab. $(4,5,14$ exp.)

Mustela frenata (11)

M. vison (11)

Peromyscus maniculatus (11)

Procyon lotor (9)

Rattus sp. $(2,3)$

Sorex vagrans (11)

Spermophilus columbianus (11)

Tamiasciurus hudsonicus (11)

Ursus americanus $(7,9,10,11)$

$U$. $\operatorname{arctos}(6,11)$

$U$. maritimus $(12,14)$

Vulpes vulpes $(9,14)$

Lab. rat (1)

Dist.: Alta, BC, NB, Nfld, NS, NWT, PEI, Que, YT

Records: 1. Moynihan and Musfeldt 1949 (BC);

2. Frank 1951 (NB, NS, PEI); 3. Pool 1952

(BC); 4. Shanta and Meerovitch 1967a exp.

(Que); 5. Shanta and Meerovitch 1967b (Que);

6. Choquette et al. 1969 (NWT, YT); 7. Schmitt et al. 1972 (BC); 8. Choquette et al. 1973

(NWT, YT); 9. Frechette and Panisset 1973

(Que); 10. Frechette and Rau 1977 (Que); 11.

Schmitt et al. 1978 (BC); 12. Smith 1978a

(Nfld); 13. Dies 1980 (Alta); 14. Chadee and

Dick 1982 (Man, NWT); 15. Bourque 1985

(Que)

Remarks: Smith (1978a) reported T. spiralis

from Thalarctos maritimus. I am following

Hall's (1981) classification and calling the host

Ursus maritimus.

Trichinella spiralis var. pseudospiralis

Site: muscle

Hosts: Mus musculus $(1,2)$

Procyon lotor (1)

Dist.: Man

Records: 1. Dick 1983 exp.; 2. Dick and Chadee 1983

Trichinella spp.

Site: unspecified

Hosts: Canis lupus (1)

Procyon lotor (2)

Lab. mouse (2)

Dist.: Alta, Man

Records: 1. Gunson and Dies 1980 (Alta); 2.

Dick 1983 exp. (Man)

Trichinella spp. larva

Site: diaphragm, tongue

Hosts: Felis concolor (3)

Martes americana (2)

Ursus americanus (1)

$U$. $\operatorname{arctos}(3)$

Dist.: Alta, Man, Ont
Records: 1. Addison et al. 1978 (Ont); 2. Poole et al. 1983 (Man); 3. Dies and Gunson 1984

(Alta)

\section{FAMILY TRICHURIDAE}

Capillaria aerophila Creplin, 1839

Sites: Lungs, bronchi, trachea

Hosts: Canis latrans (3)

C. lupus (3)

Martes americana $(5,6)$

Vulpes vulpes $(1,2,4,5,6)$

Dist.: Alta, NB, NS, Ont, Que

Records: 1. Law and Kennedy 1932 (Ont); 2.

Swales 1933b (Que); 3. Holmes and Podesta

1968 (Alta); 4. Smith 1978b (NB, NS); 5.

Butterworth and Beverley-Burton 1980 (Ont),

6. 1981 (Ont)

Capillaria eubursata Skarbilovich, 1946

Site: digestive tract

Host: Myotis lucifugus

Dist.: BC

Record: Webster and Casey 1973

Capillaria hepatica Bancroft, 1896

Site: liver

Hosts: Antilocapra americana (11)

Canis latrans (13)

Clethrionomys gapperi (10)

C. g. gapperi (8)

Microtus pennsylvanicus (10)

M. p. pennsylvanicus (8)

Mus musculus lab. (8 exp., 9 exp.,12)

Napaeozapus $i$. insignis (8)

Ondatra zibethicus $(1,2,4,5,14)$

Peromyscus leucopus (9 exp.)

P. l. noveboracensis (8 exp.)

$P$. maniculatus $(5,6,7,8,9,10)$

Rattus norvegicus (3)

Spermophilus franklinii (15)

S. richardsonii (15)

Synaptomys c. cooperi (8)

Tamiasciurus hudsonicus (8 exp.)

Thomomys talpoides $(5,6)$

Dist.: Alta, BC, Man, Ont, Que, Sask

Records: 1. Price 1931 (Ont); 2. Law and

Kennedy 1932 (Ont); 3. Firlotte 1948 (Que); 4.

Knight 1951 (BC); 5. Lubinsky 1956 (Alta), 6. 1957 (Alta); 7. Freeman 1958 (Ont); 8.

Freeman and Wright 1960 (Ont); 9. Wright 1961 (Ont); 10. Lubinsky et al. 1971 (Man); 11. Barret and Chalmers 1972 (Alta); 12. Wright 1972 (Ont); 13. Wobeser and Rock 1973 (Sask); 14. McKenzie and Welch 1979 (Man); 15. McGee 1980 (Sask) 
Capillaria michiganensis Read, 1949

Site: intestine

Hosts: Ondatra zibethicus $(1,2)$

O. z. obscurus (3)

Dist.: Man, Nfld, Ont

Records: 1. Webster 1966b (Ont); 2. McKenzie and Welch 1979 (Man); 3. Rigby and Threlfall 1981 (Nfld)

Capillaria mustelorum Cameron and Parnell, 1932

Site: intestine

Host: Mustela vison

Dist.: Nfld

Record: Jennings et al. 1982

Remarks: Jennings et al. (1982) did not specify the site in which their specimens were found, but presumably it was the intestine.

Capillaria plica (Rudolphi, 1819) Travassos, 1915

Site: urinary bladder

Hosts: Canis latrans $(3,5)$

Martes pennanti $(3,5)$

Mephitis mephitis $(3,5)$

Procyon lotor $(3,5)$

Sorex cinereus (4)

Vulpes vulpes $(1,2,3,5)$

Dist.: Ont, Que

Records: 1. Law and Kennedy 1932 (Ont); 2.

Swales 1933b (Que); 3. Butterworth and

Beverley-Burton 1980 (Ont); 4. Bourque 1981

(Que); 5. Butterworth and Beverley-Burton 1981 (Ont)

Capillaria procyonis Pence, 1975

Site: esophageal mucosa

Hosts: Mephitis mephitis

Dist.: Ont

Procyon lotor

Records: Butterworth and Beverley-Burton 1980,1981

Capillaria putorii (Rudolphi, 1819) Travassos, 1915

Site: stomach

Hosts: Martes americana

$M$. pennanti

Mephitis mephitis

Mustela erminea

$M$. vison

Procyon lotor

Dist.: Ont

Records: Butterworth and Beverley-Burton

1980,1981
Capillaria ransomia Barker and Noyes, 1915

Site: intestine

Host: Ondatra zibethicus

Dist.: BC

Record: Knight 1951

Capillaria spp.

Site: small intestine

Hosts: Canis latrans (7)

Mustela vison $(1,2)$

Odocoileus hemionus columbianus

$(3,4)$

Ovibos moschatus (6)

Peromyscus maniculatus (5)

Procyon lotor (2)

Dist.: Alta, BC, Ont, Que

Records: 1. Anonymous 1930 (Ont); 2. Law and

Kennedy 1932 (Ont); 3. Cowan 1946 (BC), 4.

1951 (BC); 5. Schad 1956 (Que); 6. Samuel and

Gray 1974 capt. (Alta); 7. Samuel,

Ramalingam et al. 1978 (Alta)

Capillaria sp.larva

Site: intestine

Host: Mustela erminea

Dist.: Nfld

Record: Jennings et al. 1982

Remarks: Jennings et al. (1982) did not specify the site in which their specimens were found,

but presumably it was the intestine.

Trichosomoides crassicauda (Bellingham, 1840) Railliet, 1895

Site: intestine

Host: Rattus norvegicus

Dist.: Que

Record: Firlotte 1948

Trichuris fossor Hall, 1916

Site: caecum

Host: Thomomys talpoides

Dist.: Alta

Record: Lubinsky 1957

Trichuris leporis (Froelich, 1789)

Site: large intestine

Hosts: Lepus americanus (1) Spermophilus richardsonii (2)

Dist.: Man

Records: 1. Boughton 1932 ; 2. Tiner 1950

Trichuris muris (Schrank, 1788) Hall, 1916

Sites: caecum, colon

Host: Mus musculus

Dist.: Ont

Record: Lee and Wright 1978 exp. 
Trichuris opaca Barker and Noyes, 1915

Site: caecum

Host: Ondatra zibethicus

Dist.: BC, Ont

Records: Knight 1951 (BC); Sweatman 1952b

(Ont)

Trichuris oreamnos Knight, 1974

Site: caecum

Host: Oreamnos americanus

Dist.: BC

Records: Knight 1974; Samuel, Hall et al. 1978

Trichuris ovis (Abildgaard, 1795) Smith, 1908

Sites: caecum, ileum

Hosts: Cervus elaphus (5)

Odocoileus hemionus columbianus

$(3,4)$

Oreamnos americanus $(2,4)$

Ovis c. canadensis (1)

Dist.: Alta, BC

Records: 1. Cowan 1942 (BC), 2. 1944 (Alta), 3. 1946 (BC), 4. 1951 (Alta, BC); 5. Flook and

Stenton 1969 (Alta)

Trichuris schumakovitschi (Savinkova, 1967)

Knight and Uhazy, 1973

Site: intestine

Hosts: Oreamnos americanus (2)

Ovis c. canadensis $(1,2)$

Dist.: Alta, Canada

Records: 1. Knight and Uhazy 1973 (Canada);

2. Samuel, Hall et al. 1978 (Alta)

Trichuris vulpis (Froelich, 1789)

Site: intestine

Host: Vulpes vulpes

Dist.: Que

Record: Swales 1933b

Remarks: Swales (1933b) did not specify the site in which his specimens were found, but presumably it was the intestine.

Trichuris spp.

Sites: caecum, feces

Hosts: Alces alces $(2,8,11)$

A. a. andersoni (5)

Cervus elaphus (11)

Odocoileus hemionus (9)

Oreamnos americanus $(3,9)$

Ovibos moschatus $(6,7)$

Ovis canadensis californianus (2)

Spermophilus richardsonii $(1,10)$

Dist.: Alta, BC, Man, Sask

Records: 1. Rausch and Tiner 1948 (Man); 2.

Blood 1963 (BC); 3. Kerr and Holmes 1966

(Alta); 4. Walker and Becklund 1970 (BC); 5.
Barrett 1972 (Alta); 6. Samuel and Gray 1974 capt. (Alta); 7. MacDonald et al. 1976 (Alta); 8. Samuel et al. 1976 (Alta); 9. Samuel, Hall et al. 1978 (Alta); 10. McGee 1980 (Sask); 11. Stock and Barrett 1983 (Alta) Remarks: Kerr and Holmes (1966) feel their species was probably Trichuris ovis.

\section{SUBCLASS SECERNENTEA ORDER ASCARIDIDA SUPERFAMILY ASCARIDOIDEA}

\section{FAMILY ANISAKIDAE}

Anisakis sp.

Site: stomach

Host: Guinea pig

Dist.: Que

Record: Myers 1963 exp.

\section{FAMILY ASCARIDIDAE}

Ascaris spp.

Site: intestine

Hosts: Mustela vison (2)

Procyon lotor $(1,2)$

Tamiasciurus hudsonicus (4)

Taxidea taxus (3)

Ursus americanus (5)

Dist.: Alta, Ont, Que

Records: 1. Anonymous 1930 (Ont); 2. Law and Kennedy 1932 (Ont); 3. Swales 1933b (Alta); 4. Mahrt and Chai 1972 (Alta); 5. Juniper 1978 (Que)

Baylisascaris columnaris (Leidy, 1856)

Syn.: Ascaris columnaris

Site: digestive tract

Host: Mephitis mephitis

Dist.: Ont, Que

Records: Choquette 1951 (Que); Sprent 1952

(Ont); Webster and Wolfgang 1956 (Que)

Baylisascaris devosi (Sprent, 1952)

Syn.: Ascaris devosi

Site: intestine

Hosts: Gulo gulo (3)

Martes americanus (4)

M. a. americanus (1)

$M$. pennanti (4)

M. p. pennanti (1)

Mephitis mephitis (1 exp.)

Mustela putorius (1 exp.)

Dist.: Man, NWT, Ont

Records: 1. Sprent 1952 (Ont); 2. Addison and Boles 1978 (NWT); 3. Dick and Leonard 1979 (Man); 4. Poole et al. 1983 (Man) 
Baylisascaris laevis (Leidy, 1856)

Site: small intestine

Hosts: Marmota vancouverensis (2)

Spermophilus richardsonii (1)

Dist.: BC, Sask

Records: 1. McGee 1980 (Sask); 2. Mace and

Shepard 1981 (BC)

Baylisascaris transfuga (Rudolphi, 1819) Sprent, 1968

Syn.: Ascaris transfuga Rudolphi, 1819

Sites: large and small intestine

Hosts: Ursus americanus $(4,5,6)$

U. a. vancouveri (2)

$U . \operatorname{arctos}(1,3)$

Dist.: Alta, BC, NWT, Ont, Que, YT

Records: 1. Baird 1853 (BC); 2. Cowan 1942

(BC); 3. Choquette et al. 1969 (YT?, NWT?); 4.

Frechette and Rau 1977 (Que); 5. Addison et al. 1978 (Ont); 6. Dies 1979 (Alta)

Remarks: Baird (1853) referred to the grizzly bear as Ursus ferox.

Baylisascaris transfuga (Rudolphi, 1819) Sprent, 1968

(eggs)

Site: intestine

Host: Ursus americanus

Dist.: Que

Record: Frechette and Rau 1978

Baylisascaris sp.larva

Site: liver

Host: Spermophilus richardsonii

Dist.: Sask

Record: McGee 1980

Toxascaris leonina (Von Linstow, 1902) Leiper, 1907

Syn.: Toxascaris limbata

Site: small intestine

Hosts: Alopex lagopus (9)

Canis latrans $(5,8)$

C. lupus $(5,7,8)$

Lynx canadensis $(4,6)$

L. rufus fasciatus (3)

Vulpes vulpes $(1,2,8)$

Dist.: Alta, BC, Man, Nfld, NWT, Ont, Que, YT

Records: 1. Law and Kennedy 1932 (Ont); 2. Swales 1933b (Que); 3.Cowan 1942 (BC); 4.

Zyll de Jong 1966 (Alta, NWT); 5. Holmes and

Podesta 1968 (Alta); 6. Threlfall 1969 (Nfld); 7.

Choquette et al. 1973 (NWT, YT); 8. Samuel, Ramalingam et al. 1978 (Man); 9. Eaton and

Secord 1979 (NWT)
Toxocara canis (Werner, 1782) Stiles, 1905

Site: small intestine

Hosts: Canis latrans (2)

C. lupus (2)

Vulpes vulpes $(1,3,4)$

Dist.: Alta, Man, NB, NS, Ont

Records: 1. Law and Kennedy 1932 (Ont); 2.

Holmes and Podesta 1968 (Alta); 3. Baron

1970 (Man); 4. Smith 1978b (NB, NS)

Toxocara cati (Schrank, 1788) Brumpt, 1927

Syn: Toxocara mystax (Zeder, 1800)

Site: small intestine

Host: Lynx canadensis

Dist.: Alta, NWT, Ont

Record: Anonymous 1930 (Ont); Law and

Kennedy 1932 (Ont); Zyll de Jong 1966 (Alta, NWT)

Ascarid gen. sp. egg

Site: intestine (feces)

Host: Ursus americanus

Dist.: Que

Record: Frechette and Rau 1977

Remarks: Frechette and Rau (1977) noted that their specimens were probably Baylisascaris transfuga.

\section{SUPERFAMILY HETERAKOIDEA}

\section{FAMILY HETERAKIDAE}

Heterakis spumosa Schneider, 1866

Site: intestine

Host: Rattus norvegicus

Dist.: Nfld, Que

Records: Firlotte 1948 (Que); Smith and

Threlfall 1973 (Nfld)

\section{SUPERFAMILY SUBULUROIDEA}

FAMILY SUBULURIDAE

Oxynema sp.

Site: small intestine

Host: Spermophilus tridecemlineatus

Dist.: Alta

Record: Lubinsky 1957

ORDER OXYURIDA SUPERFAMILY OXYUROIDEA

FAMILY HETEROXYNEMATIDAE 
Cephaluris alaskensis Akhtar

Site: gastrointestinal tract

Hosts: Ochotona collaris

O. princeps

Dist.: Alta, YT

Record: Hobbs 1980

Cephaluris coloradensis Olsen, 1949

Site: gastrointestinal tract

Hosts: Ochotona collaris

O. princeps

Dist.: Alta, YT

Record: Hobbs 1980

Heteroxynema cucullatum Hall, 1916

Site: caecum

Host: Eutamias minimus

Dist.: Sask

Record: McGee 1980

Labiostomum coloradensis Leiby, 1961

Site: gastrointestinal tract

Host: Ochotona princeps

Dist.: Alta

Record: Hobbs 1980

Labiostomum rauschi Akhtar, 1956

Site: gastrointestinal tract

Host: Ochotona collaris

Dist.: YT

Record: Hobbs 1980

Labiostomum talkeetnaeuris (Akhtar, 1956)

Syn.: Eugenuris talkeetnaeuris Akhtar, 1956

Site: gastrointestinal tract

Host: Ochotona collaris

Dist.: YT

Record: Hobbs 1980

Unidentified Labiostomum sp.

Site: gastrointestinal tract

Host: Ochotona princeps

Dist.: Alta

Record: Hobbs 1980

\section{FAMILY OXYURIDAE}

Passalurus nonanulatus Skinker, 1931

Site: large intestine

Host: Lepus americanus

Dist.: Man

Record: Boughton 1932
Skrjabinema ovis Skrjabin, 1915

Site: caecum, large intestine

Hosts: Oreamnos americanus $(1,2)$

Ovis c. canadensis (2)

Dist.: Alta

Records: 1. Kerr and Holmes 1966; 2. Samuel,

Hall et al. 1978

Skrjabinema sp.

Sites: caecum, large intestine

Host: Ovis canadensis californianus

Dist.: BC

Record: Blood 1963

Syphacia citelli Tiner and Rausch, 1950

Site: caecum

Host: Spermophilus richardsonii

Dist.: Sask

Record: McGee 1980

Syphacia eutamii Tiner, 1948

Site: large intestine

Host: Eutamias amoenus

Dist.: Alta

Record: Lubinsky 1957

Syphacia obvelata (Rudolphi, 1802) Seurat, 1916

Syn.: Ascaris obvelata Rudolphi, 1802

Site: large intestine

Hosts: Microtus pennsylvanicus (2)

M. p. drummondi (1)

Mus musculus (3)

Dist.: Man, Nfld, Que

Records: 1. Rausch and Tiner 1949 (Man); 2.

Schad 1954 (Nfld, Que); 3. Threlfall 1969

(Nfld)

Syphacia peromysci Harkema, 1936

Sites: caecum, colon

Host: Peromyscus maniculatus

Dist.: Que

Record: Schad 1956

Syphacia thompsoni Price, 1928

Site: caecum

Host: Tamiasciurus hudsonicus

Dist.: Alta, Sask

Records: Mahrt and Chai 1972 (Alta); McGee 1980 (Sask) 
ORDER RHABDITIDA

SUPERFAMILY RHABDITOIDEA

FAMILY RHABDITIDAE

Pelodera strongyloides (Schneider, 1860)

Schneider, 1866

larva

Site: conjunctival sacs

Hosts: Dicrostonyx groenlandicus

Lemmus trimucronatus

Microtus pennsylvanicus (exp.)

Mus musculus (exp.)

Dist.: NWT

Peromyscus maniculatus (exp.)

Record: Cliff et al. 1978

\section{FAMILY STRONGYLOIDIDAE}

Strongyloides papillosus (Wedl, 1856) Ransom, 1911

Site: large intestine

Host: Lepus americanus

Dist.: Man

Record: Boughton 1932

Stongyloides ratti Sandground, 1925

Site: intestine

Host: Rattus norvegicus

Dist.: Que

Record: Firlotte 1948

Strongyloides robustus Chandler, 1942

Site: intestine

Hosts: Spermophilus richardsonii

$S$. tridecemlineatus

Tamiasciurus hudsonicus

Dist.: Sask

Record: McGee 1980

Strongyloides sp.

Site: intestine

Host: Mustela vison

Dist.: Ont

Record: Law and Kennedy 1932

ORDER SPIRURIDA

SUPERFAMILY

DRACUNCULOIDEA

FAMILY DRACUNCULIDAE
Dracunculus insignis (Leidy, 1858) Chandler, 1942

Site: connective tissue

Hosts: Martes pennanti $(4,5)$

Mephitis mephitis (2)

Mustela vison $(4,5,6)$

Ondatra zibethicus (3)

Procyon lotor $(4,5,6,7)$

Mink (1)

Muskrat (1)

Otter (1)

Raccoon (1)

Dist.: Ont

Records: 1. Fyvie 1966; 2. Webster and Casey 1970; 3. Gibson and McKiel 1972; 4. Crichton and Beverley-Burton 1973, 5. 1974, 6. 1975 exp., 7. 1977

Dracunculus lutrae Crichton and Beverley-

Burton, 1973

Site: connective tissue

Host: Lutra canadensis

Dist.: Ont

Records: Crichton and Beverley-Burton 1973, 1974

Dracunculus spp.

Site: unspecified

Hosts: Didelphis virginiana $(2,3)$

Lutra canadensis (1)

Mustela erminea $(1,2,3)$

$M$. vison (1)

Ondatra zibethicus $(1,2,3)$

Procyon lotor (3)

Taxidea taxus (2)

Dist.: Man, Ont

Records: 1. Crichton 1971 (Ont); 2. Crichton and Beverley-Burton 1973 (Man, Ont), 3. 1974

(Ont)

Remarks: Crichton (1971) stated that

Dracunculus sp. in his Ondatra zibethicus and

Mustela erminea ..."probably represent accidental infections."

SUPERFAMILY FILARIOIDEA

FAMILY FILARIIDAE

Filaria sp.

Site: trachea

Host: Mustela vison

Dist.: Ont

Record: Law and Kennedy 1932 


\section{FAMILY ONCHOCERCIDAE}

Ackertia marmotae Webster, 1967

Sites: lymphatics, connective tissue of the gall bladder and bile

Hosts: Marmota monax (2)

M. m. canadensis (1)

Dist.: Ont, Que

Records: 1. Webster 1967b (Ont, Que); 2. Ko 1972 (Ont)

Cercopithifilaria leporinus Bartlett, 1983

Site: subcutaneous tissue

Host: Lepus americanus

Dist.: Alta, Ont

Record: Bartlett 1983b

Dipetalonema mephitis Webster and Beauregard, 1964

Site: subcutaneous tissue

Host: Mephitis mephitis

Dist.: Que

Records: Webster and Beauregard 1964, 1965

Dipetalonema mephitis Webster and Beauregard, 1964

(microfilaria)

Syn.: Microfilaria mephitis Webster and Beauregard, 1964

Site: blood, brain

Hosts: Mephitis mephitis

Dist.: Ont, Que

Records: Webster and Beauregard 1964 (Que),

1965 (Que); Webster 1966a (Ont)

Dipetalonema sprenti Anderson, 1953

Site: peritoneal cavity

Host: Castor canadensis

Dist.: Ont

Records: Anderson 1953; Addison 1973

Dipetalonema sprenti Anderson,1953

(microfilaria)

Site: blood

Host: Castor canadensis

Dist.: Ont

Record: Addison 1973

Dirofilaria scapiceps (Leidy, 1886) York and Maplestone, 1926

Syn.: Filaria scapiceps Leidy, 1886

Sites: subcutaneous fascia of the shoulder and lumbar regions; intermuscular fascia of the hind legs
Hosts: Lepus americanus $(2,3,4,5)$

L. a. struthopus (1)

L. capensis (2)

Oryctolagus cuniculus $(2,3)$

Sylvilagus floridanus $(2,3,4)$

Dist.: Alta, NB, NWT, Ont

Records: 1. Maltais and Ouellette 1979 (NB);

2. Bartlett 1983a (Alta, NWT, Ont), 3. 1984a $\exp$ (Ont), 4. 1984b (Alta, Ont); 5. Keith et al. 1985 (Alta)

Dirofilaria ursi Yamaguti, 1941

Sites: superficial abdominal fascia, adipose tissue of inguinal region, oesophagus

Hosts: Ursus americanus $(4,5,6)$

$U$. a. americanus $(2,5)$

$U \cdot \operatorname{arctos}(3)$

Dist.: Alta, BC, Ont, Que, YT

Records: 1. Anderson 1952 (Ont); 2. Choquette 1952 (Ont, Que); 3. Choquette et al. 1969 (BC, YT); 4. Frechette and Rau 1977 (Que); 5.

Addison et al. 1978 (Ont); 6. Dies 1979 (Alta)

Dirofilaria ursi Yamaguti, 1941

(microfilaria)

Site: blood

Host: Ursus americanus

Dist.: Ont

Record: Addison et al. 1978; Addison 1980

Elaeophora schneideri Wehr and Dikmans, 1935

Site: large arteries

Host: Odocoileus hemionus columbianus

Dist.: BC

Record: Cowan 1946, Cowan 1951

Litomosoides carinii (Travassos, 1919) Vaz, 1934

Site: coelom

Host: Thomomys talpoides

Dist.: Alta

Record: Lubinsky 1957

Litomosoides guiterasi (Vigueras, 1934)

Sandground, 1934

Site: digestive tract

Host: Eptesicus fuscus

Dist.: BC

Record: Webster and Casey 1973 
Onchocerca cervipedis Wehr and Dikmans, 1935

Syn.: Wehrdikmansia cervipedis (Wehr and

Dikmans, 1935) Caballero, 1945

Site: subcutaneous connective tissue

Hosts: Alces alces $(2,3,6,7,8)$

A. a. andersoni (5)

Odocoileus hemionus columbianus (2)

O. h. hemionus (2)

$O$. virginianus (4)

Dist.: Alta, BC, Ont

Records: 1. Cowan 1946 (BC), 2. 1951 (BC); 3.

Ritcey and Edwards 1958 (BC); 4 . Anderson

1962b (Ont); 5. Barrett 1972 (Alta); 6. Samuel

1972b (Alta); 7. Samuel et al. 1976 (Alta); 8.

Pledger et al. 1980 (Alta)

Onchocerca sp.

Site: leg muscle

Host: Rangifer tarandus caribou

Dist.: BC

Record: Low 1976

Rumenfilaria andersoni Lankester and Snider, 1982

Site: subserosal veins of the rumen

Host: Alces alces

Dist.: Ont

Record: Lankester and Snider 1982

Setaria cervi (Dujardin, 1845) Becklund and

Walker, 1964

Site: peritoneal cavity

Hosts: Cervus elaphus (4)

Odocoileus hemionus columbianus

$(2,3)$

Rangifer tarandus (1)

Dist.: Alta, BC, Sask

Records: 1. Erickson and Highby 1942 (Sask);

2. Cowan 1946 (BC), 3. 1951 (BC); 4. Flook and

Stenton 1969 (Alta)

Setaria labiatopapillosa (Perroncito, 1882) Railliet and Henry, 1911

Site: peritoneal cavity

Hosts: Alces alces (3)

Bison bison $(1,2)$

Dist.: Alta

Records: 1. Cameron 1923; 2. Becklund and

Walker 1969; 3. Samuel et al. 1976

Setaria tundra Isaichikov and Raevskays, 1928

Site: body cavity

Host: Odocoileus virginianus

Dist.: Ont

Record: Anderson 1962b
Setaria yehi Desset, 1966

Site: peritoneal cavity

Hosts: Alces alces (2)

Odocoileus hemionus (1)

Rangifer tarandus (1)

Dist.: Alta, BC, NWT

Records: 1. Becklund and Walker 1969 (BC,

NWT); 2. Samuel et al. 1976 (Alta)

Setaria spp.

Site: intestine

Hosts: Alces alces $(1,3,4)$

A. a. andersoni (2)

Dist.: Alta, Man, Que

Records: 1. Peterson 1955 (Que); 2. Barrett

1972 (Alta); 3. Crichton 1979 (Man), 4.1980

(Man)

SUPERFAMILY

GNATHOSTOMATOIDEA

FAMILY GNATHOSTOMATIDAE

Gnathostoma miyazakii Anderson, 1964

Site: kidneys

Host: Lutra c. canadensis

Dist.: Ont

Record: Anderson 1964a

\section{SUPERFAMILY PHYSALOPTEROIDEA}

FAMILY PHYSALOPTERIDAE

Physaloptera bispiculata Vaz and Pereira, 1955

Site: stomach

Host: Ochotona princeps

Dist.: Alta

Record: Lubinsky 1957

Physaloptera maxillaris Molin, 1860

Site: digestive tract

Host: Mephitis mephitis

Dist.: Ont, Que

Records: Choquette 1951 (Que); Webster and

Beauregard 1965 (Que); Lincoln and Anderson 1973 (Ont); Cawthorn and Anderson 1976a

(Ont), 1976b (Ont)

Physaloptera praeputialis Linstow, 1899

Site: stomach

Host: Lynx canadensis

Dist.: Alta, NWT

Record: Zyll de Jong 1966 
Physaloptera rara Hall and Wigdor, 1918

Site: intestine

Host: Ursus americanus

Dist.: Ont

Record: Addison et al. 1978 capt.

Physaloptera spinicauda McLeod, 1933

Site: stomach

Hosts: Spermophilus franklinii $(1,3)$

$S$. richardsonii (3)

S. tridecemlineatus $(1,2,3)$

Dist.: Alta, Man, Sask

Records: 1. Mcleod 1933 (Man); 2. Lubinsky

1957 (Alta); 3. McGee 1980 (Sask)

Physaloptera spinicauda McLeod, 1933

(larva)

Sites: stomach, duodenum

Hosts: Spermophilus franklinii

S. richardsonii

S. tridecemlineatus

Dist.: Sask

Record: McGee 1980

Physaloptera spp.

Site: stomach, intestine

Hosts: Canis latrans (7)

Gulo gulo (6)

Lynx canadensis (2)

Martes pennanti (8)

Mephitis mephitis (4)

Procyon lotor $(1,2,3)$

P. l. hirtus (9)

Tamiasciurus hudsonicus (5)

Taxidea taxus (3)

Dist.: Alta, Man, NWT, Ont, Que, Sask

Records: 1. Anonymous 1930 (Ont); 2. Law and

Kennedy 1932 (Ont); 3. Swales 1933b (Alta,

Que); 4. Webster and Wolfgang 1956 (Que); 5.

Mahrt and Chai 1972 (Alta); 6. Addison and

Boles 1978 (NWT); 7. Samuel, Ramalingam et

al. 1978 (Alta); 8. Dick and Leonard 1979

(Man); 9. Hoberg and McGee 1982 (Sask)

Remarks: Webster and Wolfgang (1956)

believed their Physaloptera sp. was probably

$P$. maxillaris .

\section{SUPERFAMILY RICTULARIOIDEA}

\section{FAMILY RICTULARIIDAE}

Rictularia citelli McLeod, 1933

Sites: stomach, duodenum

Hosts: Spermophilus franklinii

S. tridecemlineatus

Dist.: Man

Record: McLeod 1933
Rictularia coloradensis Hall, 1916

Site: stomach

Host: Microtus pennsylvanicus

Dist.: Alta

Record: Lubinsky 1957

Rictularia lucifugus Douvres, 1956

Site: digestive tract

Hosts: Eptesicus fuscus

Myotis lucifugus

$M$. volans longicrus

Dist.: BC

Record: Webster and Casey 1973

Rictularia sp.

Site: digestive tract

Host: Myotis lucifugus

Dist.: BC

Record: Webster and Casey 1973

\section{SUPERFAMILY SPIRUROIDEA}

\section{FAMILY SPIROCERCIDAE}

Cylicospirura subaequalis (Molin, 1860)

Site: stomach

Host Lynx canadensis

Dist.: Alta, Nfld, NWT

Records: Zyll de Jong 1966 (Alta, NWT);

Threlfall 1969 (Nfld); Pence et al. 1978 (Alta)

Spirocerca arctica Petrov, 1927

Site: stomach

Host: Canis lupus

Dist.: NWT

Record: Choquette et al. 1973

Spirocerca lupi (Rudolphi, 1809) Railliet and

Henry, 1911

Sites: esophagus, stomach

Hosts: Canis lupus

Dist.: NWT

Record: Choquette et al. 1973

\section{FAMILY SPIRURIDAE}

Protospirura ascaroidea Hall, 1916

Site: stomach

Host: Thomomys talpoides

Dist.: Alta

Record: Lubinsky 1957 
Protospirura muris (Gmelin, 1780)

Syn.: Mastophorus muris (Gmelin, 1790)

Site: stomach, liver

Hosts: Clethrionomys gapperi $(2,4)$

Peromyscus maniculatus (3)

Rattus norvegicus (1)

Zapus hudsonius (2)

Dist.: Alta, Nfld, Que

Record: 1. Firlotte 1948 (Que); 2. Schad 1954

(Nfld), 3. 1956 (Que); 4.

Lubinsky 1957 (Alta)

Spirura infundibuliformis McLeod, 1933

Sites: stomach, esophagus

Hosts: Spermophilus franklinii (2)

$S$. richardsonii $(1,2)$

S. tridecemlineatus $(1,2)$

Dist.: Man, Sask

Records: 1. McLeod 1933 (Man); 2. McGee 1980 (Sask)

Spirura infundibuliformis McLeod, 1933

(larva)

Sites: stomach, esophagus

Hosts: Spermophilus franklinii

S. richardsonii

S. tridecemlineatus

Dist.: Sask

Record: McGee 1980

\section{ORDER STRONGYLIDA} SUPERFAMILY

\section{ANCYLOSTOMATOIDEA}

FAMILY ANCYLOSTOMATIDAE

Ancylostoma caninum Ercolani, 1854

Site: intestine

Hosts: Canis latrans (2)

Vulpes vulpes (1)

Dist.: Alta, Que

Records: 1. Swales 1933b (Que); 2. Holmes and

Podesta 1968 (Alta)

Arthrocephalus lotoris (Schwartz, 1925) Chandler, 1942

Site: digestive tract

Host: Mephitis mephitis

Dist.: Que

Record: Choquette 1951
Placoconus lotoris (Schwartz, 1925) Webster, 1956

Site: intestine

Hosts: Mephitis mephitis (1) Procyon lotor $(2,3)$

Dist.: Que

Records: 1. Webster and Wolfgang 1956; 2.

Balasingam 1964a exp., 3. 1964b exp.

Uncinaria yukonensis (Wolfgang, 1956)

Site: small intestine

Hosts: Ursus americanus $(1,3)$

$U . \operatorname{arctos}(2)$

Dist.: NWT, Que, YT

Records: 1. Wolfgang 1956 (YT); 2. Choquette et al. 1969 (NWT, YT); 3. Frechette and Rau 1977 (Que)

Hookworm egg

Site: feces

Host: Ursus americanus

Dist.: Que

Records: Frechette and Rau 1977; Juniper 1978

Remarks: Frechette and Rau (1977) believed their material was probably Uncinaria yukonensis.

\section{SUPERFAMILY METASTRONGYLOIDEA}

\section{FAMILY ANGIOSTRONGYLIDAE}

Aelurostrongylus pridhami Anderson, 1962

Site: lungs

Hosts: Mustela erminea (4)

$M$. vison $(2,3,4)$

M. v. vison (1)

Dist.: Nfld, Ont

Records: 1. Anderson 1962a (Ont); 2. Stockdale 1970a exp. (Ont), 3. 1970b exp. (Ont); 4.

Jennings et al. 1982 (Nfld)

Andersonstrongylus captivensis Webster, 1978

Site: lungs

Host: Mephitis mephitis

Dist.: Ont

Record: Webster 1980 exp.

Angiostrongylus vasorum (Baillet, 1866)

Kamensky, 1905

Site: unspecified

Host: Vulpes vulpes

Dist.: Nfld

Record: Smith and Threlfall 1973 
Sobolevingylus sp.

Site: lungs

Host: Martes pennanti

Dist.: Ont

Record: Craig and Borecky 1976

Trilobostrongylus bioccai Anderson, 1963

Site: lungs

Hosts: Martes pennanti (2)

$$
\text { M.p. pennanti (1) }
$$

Dist.: Ont

Records: 1. Anderson 1963b; 2. Craig and

Borecky 1976

\section{FAMILY CRENOSOMATIDAE}

Crenosoma decoratum (Creplin, 1847) Stoss, 1898

Sites: lungs and trachea

Host: Vulpes vulpes

Dist.: Ont

Record: Law and Kennedy 1932

Crenosoma goblei Dougherty, 1945

Site: bronchi, bronchioles

Host: Procyon lotor

Dist.: Ont

Record: Craig and Anderson 1972

Crenosoma hermani Anderson, 1962

Site: bronchi

Host: Mustela v. vison

Dist.: Ont

Record: Anderson 1962a; Craig and Anderson 1972

Crenosoma mephitidis Hobmaier, 1941

Sites: bronchi, bronchioles, lungs

Host: Mephitis mephitis

Dist.: Ont, Que

Records: Webster 1964 (Que); Webster and

Beauregard 1965 (Que); Craig and Anderson

1972 (Ont); Stockdale et al. 1974 exp. (Ont)

Crenosoma petrowi Morozov, 1939

Sites: bronchi, bronchioles

Hosts: Martes pennanti (2)

Taxidea taxus (1)

Ursus americanus $(3,4)$

Dist.: Ont

Records: 1. Craig and Anderson 1972; 2. Craig and Borecky 1976; 3. Addison 1978; 4. Addison et al. 1978
Crenosoma potos Buckley, 1930

Site: lungs

Host: Ursus americanus

Dist.: Ont

Record: Addison 1978; Addison et al. 1978

Crenosoma vulpis (Dujardin, 1844) Railliet, 1915

Site: lungs

Hosts: Ursus americanus $(4,5)$

Vulpes vulpes $(1,2,3,6)$

Dist.: NB, Nfld, NS, Ont

Records: 1. Threlfall 1969 (Nfld); 2. Craig and Anderson 1972 (Ont); 3. Smith and Threlfall 1973 (Nfld); 4. Addison 1978 (Ont); 5. Addison et al. 1978 (Ont); 6. Smith 1978b (NB, NS)

Crenosoma spp.

Site: lungs

Hosts: Ursus americanus (2)

Dist.: Ont

Red fox (1)

Records: 1. Law and Secord 1931; 2.Addison et al. 1978

Troglostrongylus wilsoni (Stough, 1953)

Sarmiento and Stough, 1956

Site: lungs

Host: Lynx canadensis

Dist.: Alta, NWT

Record: Zyll de Jong 1966

\section{FAMILY FILAROIDIDAE}

Filaroides canadensis Anderson, 1963

Site: lungs

Host: Lutrac. canadensis

Dist.: Ont

Record: Anderson 1963b

Filaroides martis (Werner, 1782) Dougherty, 1943

Syn.: Filaroides bronchialis (Gmelin, 1790)

Sites: bronchi, trachea

Hosts: Mephitis mephitis (3)

Mustela vison $(1,5,6,7)$

M. v. vison (2)

Mink (4)

Dist.: Ont, Que

Records: 1. Law and Kennedy 1932 (Ont); 2.

Anderson 1962a (Ont); 3. Webster and

Beauregard 1965 (Que); 4. Fyvie 1966 (Ont); 5.

Stockdale 1970b exp. (Ont); 6. Stockdale and

Anderson 1970 exp. (Ont); 7. Ko and Anderson 1972 exp. (Ont) 
Filaroides mephitis Webster, 1967

Site: lungs

Host: Mephitis mephitis

Dist.: Ont

Record: Webster 1967a

Oslerus osleri (Cobbold, 1879) Anderson, Chabaud, and Willmott, 1978

Sites: lungs, trachea

Hosts: Canis latrans $(1,2,3)$

$$
\begin{aligned}
& \text { C. lupus }(1,3) \\
& \text { Coyote } x \operatorname{Dog}(1)
\end{aligned}
$$

Dist.: Alta, Man, Ont

Records: 1. Fyvie 1966 (Ont); 2. Holmes and Podesta 1968 (Alta); 3. Samuel, Ramalingam et al. 1978 (Man)

\section{Oslerus sp.}

Site: pulmonary veins

Host: Mustela vison

Dist.: Ont

Record: Anonymous 1930

\section{FAMILY PROTOSTRONGYLIDAE}

Elaphostrongylus cervi Cameron, 1931

Sites: beneath skin, fascia of thoracic

musculature

Hosts: Alces alces (1)

Rangifer tarandus (1)

R. t. caribou (2)

Dist.: Nfld, Ont

Records: 1. Lankester 1977 exp. (Ont); 2.

Lankester and Northcott 1979 (Nfld)

Elaphostrongylus cervi Cameron, 1931

(larva)

Sites: feces, trachea

Hosts: Rangifer tarandus caribou

$$
R \text {.t. groenlandicus }
$$

Dist.: Man, Nfld

Record: Lankester and Northcott 1979

Elaphostrongylus odocoilei Hobmaier and

Hobmaier, 1934

Sites: meninges, subarachnoid space

Host: Odocoileus virginianus borealis

Dist.: Ont

Record: Anderson 1956

Orthostrongylus macrotis (Dikmans, 1931)

Dougherty and Goble, 1946

Site: lungs

Hosts: Alces alces

$$
\text { Cervus elaphus }
$$

Dist.: Alta

Record: Stock and Barrett 1983
Parelaphostrongylus andersoni Prestwood, 1972

Sites: skeletal muscles of thigh and dorsum

Host: Odocoileus virgianus

Dist.: BC

Record: Pybus and Samuel 1981 exp.

Parelaphostrongylus andersoni Prestwood, 1972 (larva)

Site: feces

Host: Odocoileus virginianus

Dist.: BC

Records: Pybus and Samuel 1981; Ballantyne and Samuel 1984

Parelaphostrongylus odocoilei (Hobmaier and Hobmaier, 1934)

Boev and Schults, 1950

Sites: skeletal muscles, larvae in feces

Hosts: Alces alces (3 exp.)

A. a. andersoni (1)

Oreamnos americana (5)

Odocoileus hemionus (4)

$O$. h. columbianus $(1,5,6)$

$O$. h. hemionus $(1,2,3,6)$

$O$. virginianus / O. hemionus hybrid (5)

Dist.: Alta, BC

Records: 1. Platt and Samuel 1978a exp.

(Alta), 2. 1978b exp.; 3. Pybus and Samuel 1980 (Alta); 4. Ballantyne and Samuel 1984

(BC); 5. Pybus, Foreyt, and Samuel 1984 (Alta, BC); 6. Shostak and Samuel 1984 exp. (Alta)

Parelaphostrongylus tenuis (Dougherty, 1945)

Pryadko and Boev, 1971

Syn.: Pneumostrongylus tenuis

Dougherty, 1945

Sites: piarachnoid and meninges of brain

Hosts: Alces alces $(2,3,12)$

A. a. americana (4)

Cervus elaphus (6)

Odocoileus $h$. hemionus (6)

$O$. virginianus $(3,7,10,11)$

O. $v$. borealis (1)

Rangifer $t$. tarandus (9)

R.t. terraenovae (8)

Guinea pig ( Cavia porcellus) (5)

Dist.: Man, NB, NS, Ont, Que, Sask

Records: 1. Anderson 1963a (Ont), 2. 1964b exp. (Ont); 3. Smith et al. 1964 (NB,NS); 4.

Anderson 1965a (Ont); 5. Anderson and

Strelive 1966a (Ont); 6. Anderson et al. 1966 exp. (Ont); 7. Anderson and Strelive 1967 exp. (Ont), 8. 1968 exp. (Ont); 9. Anderson 1971b (Ont); 10. Bindernagel and Anderson 1972a (Man, Ont, Que, Sask), 11. 1972b (Man, Ont, Que, Sask); 12. Lankester 1972 (Man) 
Parelaphostrongylus tenuis (Dougherty, 1945)

Pryadko and Boev, 1971

(larva)

Site: feces

Hosts: Alces alces (1)

Odocoileus virginianus (1)

O. v. dacotensis (3)

Rangifer tarandus caribou (2)

Dist.: Alta, NS, Ont

Records: 1. Saunders 1973 (Ont); 2. Dauphine, Jr. 1975 (NS); 3. Shostak and Samuel 1984 exp. (Alta)

Parelaphostrongylus sp.

(larva)

Site: feces

Host: Odocoileus hemionus

Dist.: Alta

Record: Samuel and Holmes 1974

Protostrongylus boughtoni Goble and Dougherty, 1943

Sites: lungs, bronchi, bronchioles

Hosts: Lepus americanus $(1,2,4,5)$

L. a. americanus (3)

Oryctolagus cuniculus lab. rabbit (3)

Dist.: Alta, Man, NB, Ont

Records: 1. Boughton 1932 (Man); 2. Goble and

Dougherty 1943 (Man, NB, Ont); 3. Kralka

and Samuel 1984 exp. (Alta); 4. Keith et al.

1985 (Alta); 5. Kralka 1985 (Que)

Protostrongylus macrotis Dikmans, 1931

Site: lungs

Host: Alces alces

Dist.: Alta

Record: Samuel et al. 1976

Protostrongylus rushi Dickmans, 1937

Site: lungs

Hosts: Oreamnos americanus (2)

Ovis c. canadensis $(1,2)$

Dist.: Alta, BC

Records: 1. Uhazy et al. 1973; 2. Samuel, Hall et al. 1978

Protostrongylus stilesi Dickmans, 1931

Site: lungs

Hosts: Oreamnos americanus $(1,2,5,6)$

Ovis canadensis $(1,3)$

O. c. canadensis $(2,4,5)$

Dist.: Alta, BC

Records: 1. Cowan 1944 (Alta), 2. 1951 (Alta,

BC); 3. Hudson et al. 1970 (BC?); 4. Uhazy et al. 1973 (Alta, BC); 5. Samuel, Hall et al. 1978 (Alta); 6. Pybus, Foreyt, and Samuel 1984

(Alta)

\section{Protostrongylus sp.}

Site: lungs

Host: Oreamnos americanus

Dist.: Alta

Record: Kerr and Holmes 1966

Remarks: This parasite was reported by Kerr and Holmes (1966) as probably being

Protostrongylus stilesi.

Protostrongylus sp. larva

Site: feces, liver

Hosts: Ovis canadensis californiana (1)

$O$. c. canadensis $(2,4)$

Rangifer tarandus caribou (3)

Dist.: Alta, BC, Man, Ont

Records: 1. Blood 1963 (BC); 2. Uhazy et al. 1973

(Alta, BC); 3. Lankester et al. 1976 (Man, Ont); 4.

Gates and Samuel 1977 (Alta)

Varestrongylus alpenae (Dikmans, 1935)

Dougherty, 1945

Site: lungs

Hosts: Odocoileus h. hemionus

Dist.: Sask

$O$. virginianus

Records: Gray et al. 1985

\section{FAMILY SKRJABINGYLIDAE}

Skrjabingylus chitwoodorum Hill, 1939

Site: nasal sinuses

Hosts: Mephitis mephitis (2)

Spilogale putorius gracilis (1)

Dist.: BC, Ont

Records: 1. Cowan 1942 (BC); 2. Lankester and Anderson 1971 exp. (Ont)

Skrjabingylus lutrae Lankester and Crichton, 1972

Site: frontal sinuses

Host: Lutra canadensis

Dist.: Ont

Record: Lankester and Crichton 1972

Skrjabingylus magnus Webster, 1965

Site: frontal sinuses

Host: Mephitis mephitis

Dist.: Que

Record: Webster 1965 
Skrjabingylus nasicola (Leukart, 1842) Petrov, 1927

Site: nasal sinus

Hosts: Mustela erminea $(2,5)$

M. e. anguinae (1)

M. e. richardsonii (4)

$M$. frenata longicauda (4)

M. vison (3)

Dist.: BC, Man, Nfld, Ont

Records: 1. Swales 1938 (BC); 2. Cowan 1942

(BC); 3. Lankester and Anderson 1971 exp.

(Ont); 4. Gamble and Riewe 1982 (Man); 5.

Jennings et al. 1982 (Nfld)

Skrjabingylus sp.

Site: unspecified

Host: Mephitis mephitis

Dist.: Que

Record: Webster and Beauregard 1965

Unidentified Metastrongyloidea

Metastrongyloidea gen. spp. (larva)

Site: liver

Hosts: Blarina brevicauda

Peromyscus maniculatus

Sorex fumeus

Tamias striatus

Dist.: Ont

Record: Lankester and Anderson 1966

\section{SUPERFAMILY STRONGYLOIDEA}

\section{FAMILY CHABERTIIDAE}

Castorstrongylus castoris Chapin, 1925

Sites: caecum, colon, stomach

Host: Castor canadensis

Dist.: Alta, NB, Nfld

Records: Choquette and Pimlott 1956 (Nfld); Smith 1967 (NB); Threlfall 1969 (Nfld); Bush and Samuel 1981 (Alta)

Oesophagostomum venulosum (Rudolphi, 1809)

Railliet and Henry, 1913

Site: caecum

Hosts: Odocoileus hemionus columbianus

$(1,2)$

O. virginianus (3)

Dist.: BC, Ont

Records: 1. Cowan 1946 (BC), 2. 1951 (BC); 3.

Baker and Pursglove 1976 (Ont)
Oesophagostomum sp.

Site: intestine

Host: Bison bison

Dist.: Alta

Record: Cameron 1923

\section{SUPERFAMILY \\ TRICHOSTRONGYLOIDEA}

FAMILY AMPHIBIOPHILIDAE

Graphidiella ochotonae Olsen, 1948

Site: gastrointestinal tract

Host: Ochotona princeps

Dist.: Alta

Record: Hobbs 1980

FAMILY DICTYOCAULIDAE

Dictyocaulus filaria (Rudolphi, 1809) Railliet and Henry, 1907

Site: lungs

Host: Bison bison

Dist.: Alta

Record: Cameron 1923

Dictyocaulus hadweni Chapin, 1925

Site: lungs

Hosts: Alces alces $(2,3,4)$

Bison bison (1)

Dist.: Alta, Nfld

Records: 1. Chapin 1925 (Alta); 2. Benson 1958

(Alta); 3. Threlfall 1967 (Nfld), 4.1969 (Nfld)

Remarks: Dikmans (1936), Olsen and

Fenstermacher (1942), and Threlfall (1967)

consider this species a synonyn of Dictyocaulus viviparus.

Dictyocaulus viviparus (Bloch, 1782) Railliet and Henry, 1907

Sites: lungs, bronchioles

Hosts: Alces alces $(4,7,12,15,16)$

A. a. andersoni (13)

Cervus elaphus $(2,4,11,16)$

Odocoileus hemionus (2)

O. h. columbianus $(3,4)$

O. h. hemionus (4)

$O$. virginianus (10)

Ovibos moschatus $(6,8,14)$

Ovis canadensis (2)

$O$. c. canadensis (4)

Rangifer tarandus (9)

R.t. greonlandicus (5)

Dist.: Alta, BC, NWT, Ont, Que, Sask

Records: 1. Erickson and Highby 1942 (Sask);

2. Cowan 1944 (Alta), 3. 1946 (BC), 4.1951

(Alta, BC); 5. Banfield 1954 (NWT); 6. Durrell 
and Bolton 1957 (NWT); 7. Benson 1958

(Alta); 8. Gibbs and Tener 1958 (NWT); 9.

Gibbs 1960 (NWT?); 10.Anderson 1962b (Ont);

11. Flook and Stenton 1969 (Alta, BC);

12.Gupta and Gibbs 1971 (Que); 13. Barrett

1972 (Alta); 14. Samuel and Gray 1974 (NWT);

15.Samuel et al. 1976 (Alta); 16. Stock and

Barrett 1983 (Alta)

Dictyocaulus spp.

Sites: lung alveoli, bronchioles

Hosts: Rangifer tarandus caribou (1)

Alces alces $(2,3)$

Dist.: BC, Man

Records: 1. Low 1976 (BC); 2. Crichton 1979

(Man), 3. 1980 (Man)

Remarks: Low (1976) believed his

Dictyocaulus sp. was probably $D$. viviparus.

\section{FAMILY HELIGMOSOMIDAE}

Citellinema bifurcatum Hall, 1916

Syn.: Warrenius bifurcatus (Hall, 1916)

Sleggs, 1925

Site: intestine

Hosts: Eutamias minimus (5)

Spermophilus franklinii (5)

S. richardsonii $(1,2,3,5)$

$S$. tridecemlineatus (5)

Tamiasciurus hudsonicus $(4,5)$

T. h. hudsonicus (3)

Dist.: Alta, Man, Sask

Records: 1. Sleggs 1925 (Sask); 2. McLeod 1933

(Man, Sask); 3. Rausch and Tiner 1948 (Man);

4. Mahrt and Chai 1972 (Alta); 5. McGee 1980

(Sask)

Heligmosomoides polygyrus Hall, 1916

Syn.: Nematospiroides dubius Baylis, 1926

Site: small intestine

Hosts: Microtus chrotorrhinus (1)

Phenacomys intermedius (2)

Dist.: Alta, Nfld, Que

Records: 1. Schad 1954 (Nfld, Que); 2.

Lubinsky 1957 (Alta)

Heligmosomum carolinensis (Dikmans, 1940)

Skrjabin, 1971

Syn: Nematospiroides carolinensis

Dikmans, 1940

Site: unspecified

Hosts: Clethrionomys gapperi (1)

Ondatra zibethicus (2)

Dist.: Man, Nfld, Que

Records: 1. Schad 1954 (Nfld, Que); 2.

McKenzie and Welch 1979 (Man)
Heligmosomum hudsonius Cameron, 1937

Site: unspecified

Host: Dicrostonyx hudsonius

Dist.: Nfld, Que

Record: Schad 1954

Heligmosomum muris Yokogawa, 1920

Syn.: Nippostrongylus muris (Yokogawa, 1920)

Site: intestine

Host: Rattus norvegicus

Dist.: Que

Record: Firlotte 1948

\section{FAMILY MOLINEIDAE}

Molineus sp.

Site: unspecified

Host: Martes pennanti

Dist.: Man

Record: Dick and Leonard 1979

Murielus harpespiculus Dickmans, 1939

Site: gastrointestinal tract

Host: Ochotona princeps

Dist.: Alta

Record: Hobbs 1980

Nematodirella alcidis (Dikmans, 1935) Ivaskin, 1954

Site: small intestine

Hosts: Alces alces

Cervus elaphus

Dist.: Alta

Record: Stock and Barrett 1983

Nematodirella longispiculata Yorke and

Maplestone, 1926

Site: small intestine

Hosts: Alces alces (1,2 Alta,5,6,10)

A. a. andersoni (7)

Cervus elaphus (1)

Odocoileus hemionus columbianus (2)

Ovibos moschatus $(3,8)$

Rangifer tarandus (4)

R. t. caribou (9)

Dist.: Alta, BC, Nfld, NWT

Records: 1. Cowan 1944 (Alta), 2. 1951 (Alta, BC); 3. Gibbs and Tener 1958 (NWT); 4. Gibbs 1960 (NWT?); 5. Threlfall 1967 (Nfld), 6. 1969 (Nfld); 7. Barrett 1972 (Alta); 8. Samuel and Gray 1974 (NWT); 9. Low 1976 (BC); 10.

Samuel et al. 1976 (Alta)

Remarks: Low (1976) reported it as

Nematodirella longissimespiculata ( N. $l$.

longispiculata). 
Nematodirella longispiculata antilocaprae

Dikmans, 1935

Site: intestine

Host: Odocoileus hemionus columbianus

Dist.: BC

Record: Cowan 1946

Nematodirella sp.

Site: feces

Host: Ovibos moschatus

Dist.: Alta, NWT

Record: Samuel and Gray 1974 (Alta capt., NWT)

Nematodirus archari Sokolova, 1948

Site: small intestine

Host: Ovis c. canadensis

Dist.: Alta

Record: Samuel, Hall et al. 1978

Nematodirus davtiani Grigoryan, 1949

Site: small intestine

Hosts: Oreamnos americanus

Ovis c. canadensis

Dist.: Alta

Record: Samuel, Hall et al. 1978

Nematodirus filicollis Rudolphi, 1802

Site: small intestine

Hosts: Odocoileus hemionus columbianus

$(2,3)$

Oreamnos americanus $(1,3)$

Ovis canadensis (1)

O. c. canadensis (3)

Dist.: Alta, BC

Records: 1. Cowan 1944 (Alta), 2. 1946 (BC), 3. 1951 (Alta, BC)

Nematodirus helvetianus May, 1920

Site: small intestine

Hosts: Cervus elaphus (4)

Oreamnos americanus (2)

Ovibos moschatus $(1,3)$

Dist.: Alta, NWT

Records: 1. Samuel and Gray 1974 (NWT); 2.

Samuel, Hall et al. 1978 (Alta); 3. Webster and

Rowell 1980 (NWT); 4. Stock and Barrett 1983

(Alta)

Nematodirus maculosus Becklund, 1965

Site: small intestine

Host: Oreamnos americanus

Dist.: Alta, BC

Records: Becklund 1965 (Alta); Kerr and

Holmes 1966 (Alta); Samuel, Hall et al. 1978

(Alta, BC)
Nematodirus odocoilei Becklund and Walker, 1967

Site: small intestine

Hosts: Odocoileus hemionus (1)

$O$. virginianus (2)

Dist.: BC, Que

Records: 1. Becklund and Walker 1967 (BC); 2.

Webster and MacKay 1969 (Que)

Nematodirus oiratianus Raevskaia, 1929

Site: small intestine

Host: Ovis c. canadensis

Dist.: Alta

Record: Samuel, Hall et al. 1978

Nematodirus spathiger (Railliet, 1896) Railliet and Henry, 1909

Site: small intestine

Host: Ovis canadensis canadensis

Dist.: Alta

Record: Samuel, Hall et al. 1978

Nematodirus triangularis Boughton, 1932

Site: duodenum

Host: Lepus americanus

Dist.: Man, Nfld

Record: Boughton 1932 (Man); Smith and

Threlfall 1973 (Nfld)

Nematodirus spp.

Sites: small intestine, feces

Hosts: Alces alces (2)

Oreamnos americanus (5)

Ovibos moschatus (3)

Ovis c. canadensis $(1,5)$

Rangifer tarandus caribou (4)

Dist.: Alta, BC, NWT, Ont, Que

Records: 1. Cowan 1942 (BC); 2. Peterson 1955

(Ont, Que); 3. Samuel and Gray 1974 (NWT,

Que capt., Alta capt.); 4. Low 1976 (BC); 5.

Samuel, Hall et al. 1978 (Alta, BC)

Nematodirus spp. larva

Site: feces

Host: Ovis canadensis californianus

Dist.: BC

Record: Blood 1963

\section{FAMILY ORNITHOSTRONGYLIDAE}

Allintoshius nycticeius Chitwood, 1937

Site: digestive tract

Host: Myotis volans longicrus

Dist.: BC

Record: Webster and Casey 1973 


\section{FAMILY TRICHOSTRONGYLIDAE}

Cooperia oncophora (Railliet, 1898) Ransom, 1907

Site: small intestine

Host: Cervus elaphus

Dist.: Alta

Record: Stock and Barrett 1983

Haemonchus contortus (Rudolphi, 1803) Cobbold, 1898

Site: abomasum

Hosts: Odocoileus hemionus columbianus (1) Ovibos moschatus $(2,3)$

Dist.: Alta, BC, NWT

Records: 1. Cowan 1951 capt. (BC); 2. Durrell and Bolton 1957 (NWT); 3. MacDonald et al.

1976 capt. (Alta)

Haemonchus ostertagi [SIC]

Site: intestine

Host: Bison bison

Dist.: Alta

Record: Cameron 1923

Haemonchus placei (Place, 1893)

Site: abomasum

Host: Ovibos moschatus

Dist.: NWT

Record: Durrell and Bolton 1957

Marshallagia marshalli (Ransom, 1907) Orlov, 1933

Sites: small intestine, abomasum

Hosts: Oreamnos americanus $(2,3)$

Ovibos moschatus (4)

Ovis canadensis californianus $(1,3)$

Dist.: Alta, BC, NWT

Records: 1. Blood 1963 (BC); 2. Kerr and

Holmes 1966 (Alta); 3. Samuel, Hall et al.

1978 (Alta, BC); 4. Webster and Rowell 1980

(NWT)

Marshallagia spp.

Site: abomasum

Hosts: Oreamnos americanus (2)

Ovibos moschatus (1)

Ovis c. canadensis (2)

Dist.: Alta, BC, NWT, Que

Records: 1. Samuel and Gray 1974 (Alta capt., NWT, Que capt.); 2. Samuel, Hall et al. 1978 (Alta, BC)
Obeliscoides cuniculi Graybill, 1924

Site: stomach

Hosts: Lepus americanus $(1,3)$

L. a. struthopus (2)

Dist.: Alta, Nfld

Records: 1. Lubinsky 1957 (Alta); 2. Dodds and Mackiewicz 1961 (Nfld); 3. Keith et al. 1985

(Alta)

Obeliscoides cuniculi cuniculi Graybill, 1926

Site: stomach

Hosts: Lepus capensis (1)

Marmota monax (1)

Oryctolagus cuniculus (3)

Dist.: Ont

Sylvilagus floridanus $(1,2)$

Records: 1. Measures and Anderson 1983a

(Ont), 2. 1983b (Ont), 3. 1984 exp. (Ont)

Obeliscoides cuniculi multistriatus Measures and Anderson, 1983

Site: stomach

Hosts: Lepus americanus $(1,2)$

Oryctolagus cuniculus (3)

Dist.: Alta, Ont

Records: 1. Measures and Anderson 1983a

(Alta, Ont), 2. 1983b (Alta, Ont), 3. 1984 exp.

(Ont)

Ostertagia bisonis Chapin, 1925

Sites: stomach, duodenum

Host: Bison bison

Dist.: Alta

Record: Chapin 1925

Ostertagia circumcincta (Stadelmann, 1894)

Ransom, 1907

Site: abomasum

Hosts: Odocoileus hemionus columbianus

$(2,3)$

Oreamnos americanus $(1,3,6,8)$

Ovibos moschatus (4)

Ovis canadensis (1)

O. c. californianus (5)

O. c. canadensis $(3,8)$

Rangifer tarandus caribou (7)

Dist.: Alta, BC, NWT

Records: 1. Cowan 1944 (Alta), 2. 1946 (BC), 3.

1951 (Alta, BC); 4. Gibbs and Tener 1958

(NWT); 5. Blood 1963 (BC); 6. Kerr and

Holmes 1966 (Alta); 7. Low 1976 (BC); 8.

Samuel, Hall et al. 1978 (Alta, BC) 
Ostertagia dikmansi Becklund and Walker, 1968

Site: abomasum

Host: Odocoileus virginianus

Dist.: Ont

Records: Becklund and Walker 1968; Baker and Anderson 1975

Ostertagia marshalli Ransom, 1907

Sites: intestine, stomach

Hosts: Oreamnos americanus $(1,2)$

Ovis canadensis (1)

O. c. canadensis (2)

Dist.: Alta, BC

Records: 1. Cowan 1944 (Alta), 2. 1951 (Alta, $\mathrm{BC})$

Ostertagia mossi Dikmans, 1931

Site: abomasum

Host: Odocoileus virginianus

Dist.: Ont

Record: Baker and Anderson 1975

Ostertagia occidentalis Ransom, 1907

Site: abomasum

Hosts: Oreamnos americanus $(1,2,4,5)$

Ovis canadensis (1)

$O$. c. californianus (3)

O. c. canadensis $(2,5)$

Dist.: Alta, BC

Records: 1. Cowan 1944 (Alta), 2. 1951 (Alta, BC); 3. Blood 1963 (BC); 4. Kerr and Holmes 1966 (Alta); 5. Samuel, Hall et al. 1978 (Alta)

Ostertagia odocoilei Dikmans, 1931

Site: abomasum

Host: Odocoileus virginianus

Dist.: Ont

Records: Walker and Becklund 1970; Baker and Anderson 1975

Ostertagia oreamni (Swales, 1934)

Syn.: Skrjabinema oreamni Swales, 1934

Site: intestine

Hosts: Oreamnos americanus $(1,2)$

Rangifer tarandus $(1,2)$

Dist.: Alta

Record: 1. Swales 1934; 2.Cowan 1951

Ostertagia ostertagi (Stiles,1892) Ransom, 1907

Site: abomasum

Host: Ovis canadensis californianus

Dist.: BC

Record: Blood 1963
Ostertagia trifurcata Ransom, 1907

Site: small intestine

Hosts: Oreamnos americanus $(1,2,4,6)$

Ovibos moschatus (7)

Rangifer tarandus (3)

R. t. caribou (5)

Dist.: Alta, BC, NWT

Records: 1. Cowan 1944 (Alta), 2. 1951 (Alta, BC); 3. Choquette et al. 1957 (NWT); 4. Kerr and Holmes 1966 (Alta); 5. Low 1976 (BC); 6. Samuel, Hall et al. 1978 (Alta); 7. Webster and Rowell 1980 (NWT)

Ostertagia spp.

Site: abomasum

Hosts: Alces alces (6)

A. a. andersoni $(3)$
Cervus elaphus $(6)$
Oreamnos americanus (5)
Ovibos moschatus $(4)$
Ovis canadensis californiana (2)
O. c. canadensis $(5)$
Rangifer tarandus $(1,2)$

Dist.: Alta, BC, NWT, Que

Records: 1. Cowan 1944 (Alta), 2. 1951 (Alta, BC); 3.Barrett 1972 (Alta); 4. Samuel and Gray 1974 (Alta capt., NWT, Que capt.); 5. Samuel, Hall et al. 1978 (Alta, BC); 6. Stock and Barrett 1983 (Alta).

Remarks: Stock and Barrett (1983) reported Skrjabinagia sp. from this host. Skrjabinagia is regarded as a synonym of Ostertagia.

Spiculopteragia bohmi (Gebauer, 1932) Orloff, 1933

Site: abomasum

Host: Odocoileus virginianus

Dist.: Que

Record: Doster and Friend 1971

Teladorsagia davtiani Andreeva and Satubaldin, 1954

Site: abomasum

Host: Oreamnos americanus

Dist.: Alta

Records: Kerr and Holmes 1966; Samuel, Hall et al. 1978

Teladorsagia spp.

Site: abomasum

Hosts: Oreamnos americanus

Ovis c. canadensis

Dist.: Alta, BC

Record: Samuel, Hall et al. 1978 
Travassosius americanus Chapin, 1925

Sites: stomach, small intestine

Host: Castor canadensis

Dist.: Alta, NB, Nfld, NS

Records: Choquette and Pimlott 1956 (Nfld);

Smith 1967 (NB); Smith and Archibald 1967

(NS); Bush and Samuel 1978 (Alta), 1981

(Alta)

Trichostrongylus axei (Cobbold, 1879) Railliet and Henry, 1909

Sites: stomach, small intestine, abomasum

Hosts: Cervus elaphus (2)

Spermophilus richardsonii (1)

Dist.: Alta, Sask

Records: 1. McGee 1980 (Sask); 2. Stock and

Barrett 1983 (Alta)

Trichostrongylus calcaratus Ransom, 1911

Site: caecum

Host: Ondatra zibethicus obscurus

Dist.: Nfld

Record: Rigby and Threlfall 1981

Trichostrongylus longispicularis Gorden, 1933

Site: small intestine

Host: Alces alces

Dist.: Alta

Record: Stock and Barrett 1983

Trichostrongylus spp.

Site: small intestine

Hosts: Lepus americanus (1)

Odocoileus hemionus columbianus (2)

Dist.: BC, Man

Records: 1. Boughton 1932 (Man); 2. Cowan

1946 (BC)

Unidentified Trichostrongylidae

Trichostrongylidae gen. sp.

Site: intestine

Host: Alces alces

Dist.: Alta

Record: Samuel et al. 1976

Remarks: Samuel et al. (1976) did not specify the site in which their specimens were found, but presumably it was the intestine.

Unidentified Nematoda

Nematoda gen. spp.

Sites: intestine, lungs

Host: Lepus americanus

Dist.: Nfld

Record: Smith and Threlfall 1973
PHYLUM

ACANTHOCEPHALA

CLASS ARCHIACANTHOCEPHALA

ORDER GIGANTORHYNCHIDA

FAMILY GIGANTORHYNCHIDAE

Mediorhynchus robustus Van Cleave, 1916

Site: small intestine

Host: Spermophilus richardsonii

Dist.: Sask

Record: McGee 1980

\section{ORDER MONILIFORMIDA}

FAMILY MONILIFORMIDAE

Moniliformis spiradentatis McLeod, 1933

Sites: stomach, intestine

Host: Spermophilus tridecemlineatus

Dist.: Man

Record: McLeod 1933

\section{CLASS PALAEACANTHOCEPHALA ORDER ECHINORHYNCHIDA}

FAMILY ECHINORHYNCHIDAE

Echinorhynchus lateralis Leidy, 1851

Site: gastrointestinal tract

Host: Lutra canadensis

Dist.: Nfld

Record: Smith and Threlfall 1972

\section{ORDER POLYMORPHIDA}

\section{FAMILY POLYMORPHIDAE}

Polymorphus paradoxus Connell and Corner, 1957

Site: small intestine

Hosts: Castor canadensis (1)

Microtus pennsylvanicus (2)

Ondatra zibethicus (1)

Dist.: Alta

Records: 1. Connell and Corner 1957; 2. Platt 1978

Polymorphus spp.

Site: intestine

Hosts: Ondatra zibethicus

Dist.: BC, Man

Records: Knight 1951 (BC); McKenzie and

Welch 1979 (Man) 
Unidentified Acanthocephala

Acanthocephala gen. sp.

Site: intestine

Host: Lepus americanus

Dist.: Man

Record: Boughton 1932 


\section{HOST-PARASITE LIST}




\section{CLASS MAMMALIA \\ INFRACLASS METATHERIA \\ ORDER MARSUPIALIA}

FAMILY DIDELPHIDAE

Didelphis virginiana Kerr

Virginia

NEMATODA

Dracunculus sp. (Ont)

opossum

\section{INFRACLASS EUTHERIA \\ ORDER ARTIODACTYLA}

FAMILY ANTILOCAPRIDAE

Antilocapra americana (Ord)

Pronghorn

NEMATODA

Capillaria hepatica (Alta)

FAMILY BOVIDAE

Bison bison (L.)

Bison

PROTOZOA

Sarcocystis sp.cysts (Alta)

TREMATODA

Fascioloides magna (Alta, BC, Que)

CESTOIDEA

Echinococcus granulosus hydatid cyst (Alta,

NWT, Ont, Sask, YT)

Echinococcus sp. (Alta)

Moniezia sp. (Alta)

NEMATODA

Dictyocaulus filaria (Alta)

D. hadweni (Alta)

Haemonchus ostertagi (Alta)

Oesophagostomum sp. (Alta)

Ostertagia bisonis (Alta)

Setaria labiatopapillosa (Alta)

Oreamnos americanus (Blainville) Mountain goat

PROTOZOA

Sarcocystis sp. cysts (Alta)

CESTOIDEA

Anoplocephalidae (Alta, BC)

Avitellina sp. (Alta)

Cysticercus tenuicollis (Alta, BC)

Moniezia benedini (Alta)

Taenia hydatigena cysticercus (Alta)

Thysanosoma actinioides (Alta, BC)

NEMATODA

Marshallagia marshalli (Alta, BC)

Marshallagia sp. (Alta, BC)

Nematodirus davtiani (Alta)

N. filicollis (Alta)
$N$. helvetianus (Alta)

N. maculosus (Alta, BC)

Nematodirus sp. (Alta, BC)

Ostertagia circumcincta (Alta, BC)

O. marshalli (Alta, BC)

O. occidentalis (Alta, BC)

O. oreamni (Alta)

O. trifurcata (Alta, BC)

Ostertagia sp. (Alta, BC)

Parelaphostrongylus odocoilei (Alta)

Protostrongylus rushi (Alta, BC)

P. stilesi (Alta)

Protostrongylus sp. (Alta)

Skrjabinema ovis (Alta)

Teladorsagia davtiani (Alta)

Teladorsagia sp. (Alta, BC)

Trichuris oreamnos $(\mathrm{BC})$

T. ovis (Alta)

T. schumankovitschi (Alta)

Trichuris sp. (Alta)

Ovibos moschatus (Zimmermann)

Muskox

PROTOZOA

Eimeria faurei (Alta, NWT, Que)

E. granulosa (NWT)

E. moschati (Alta, NWT, Que)

E. oomingmakensis (NWT)

E. ovibovis (Alta, NWT, Que)

E. ovina (Alta, NWT, Que)

Eimeria sp. (Alta, NWT, Que)

Sarcocystis sp. cysts (NWT)

CESTOIDEA

Cysticercus tenuicollis (NWT)

Echinococcus granulosus hydatid cysts (NWT)

Moniezia expansa (NWT)

Moniezia sp. (Alta, NWT)

Taenia hydatigena cysticercus (Alta, NWT)

NEMATODA

Capillaria sp. (Alta)

Dictyocaulus viviparus (NWT)

Haemonchus contortus (Alta, NWT)

H. placei (NWT)

Marshallagia marshalli (NWT)

Marshallagia sp. (Alta, NWT, Que)

Nematodirella longispiculata (NWT)

Nematodirella sp.(Alta, NWT)

Nematodirus helvetianus (NWT)

Nematodirus sp. (Alta, NWT, Que)

Ostertagia circumcincta (NWT)

O. trifurcata (NWT)

Ostertagia sp. (Alta, NWT, Que)

Trichuris sp. (Alta)

Ovis canadensis Shaw

Mountain

PROTOZOA

sheep

Sarcocystis sp. cysts (Alta)

CESTODA 
Cysticercus tenuicollis (Alta)

Moniezia benedini (Alta)

NEMATODA

Dictyocaulus viviparus (Alta)

Nematodirus filicollis (Alta)

Ostertagia circumcincta (Alta)

O. marshalli (Alta)

O. occidentalis (Alta)

Protostrongylus stilesi (Alta, BC)

Ovis canadensis californiana

PROTOZOA

Eimeria sp. (BC)

NEMATODA

Marshallagia marshalli (Alta, BC)

Nematodirus sp. larva (BC)

Ostertagia circumcincta $(\mathrm{BC})$

O. occidentalis $(\mathrm{BC})$

O. ostertagi $(\mathrm{BC})$

Ostertagia sp. (BC)

Protostrongylus larva (BC)

Skrjabinema sp. (BC)

Trichuris sp. (BC)

Ovis canadensis canadensis Shaw

PROTOZOA

Eimeria ahsata (Alta, BC)

E. crandallis (Alta, BC)

E. faurei (Alta)

E. granulosa (Alta)

E. intricata (Alta)

E. ninakohlyakimovae (Alta, BC)

E. ovina (Alta, BC)

E. parva (Alta, BC)

CESTOIDEA

Cysticercus tenuicollis (Alta, BC)

Moniezia benedini (Alta, BC)

M. expansa (Alta)

Taenia hydatigena cysticercus (Alta)

Thysanosoma actinioides (BC)

NEMATODA

Dictyocaulus viviparus (Alta)

Marshallagia sp. (Alta)

Nematodirus archari (Alta)

N. davtiani (Alta)

$N$. filicollis (Alta)

$N$. oiratianus (Alta)

N. spathiger (Alta)

Nematodirus sp. (Alta, BC)

Ostertagia circumcincta (Alta, BC)

O. marshalli (Alta, BC)

O. occidentalis (Alta, BC)

Ostertagia sp. (Alta)

Protostrongylus rushi (Alta, BC)

P. stilesi (Alta, BC)

Protostronylus sp. larvae (Alta, BC)

Skrjabinema ovis (Alta)
Teladorsagia sp. (Alta)

Trichuris ovis $(\mathrm{BC})$

T. schumakovitschi (Alta, Can)

Ovis dalli Nelson

CESTOIDEA

Wyominia tetoni (YT)

\section{FAMILY CERVIDAE}

Douglas

Alces alces (L.)

Moose

PROTOZOA

Sarcocystis sp. cysts (Alta, Ont)

TREMATODA

Fascioloides magna (Alta, BC, Man, Ont,

Sask)

Paramphistomum cervi (Nfld, Ont, Que)

Paramphistomum spp. (Alta)

Zygocotyle lunata (Alta)

CESTOIDEA

Cestoidea gen. spp. (BC)

Cysticercus tenuicollis (Alta, BC, Nfld)

Cysticercus sp. cyst (BC, NS, Ont, Sask, YT)

Echinococcus granulosus hydatid cyst (Alta,

BC, Man, NS, NWT, Ont, Que, Sask, YT)

Moniezia benedini (Alta, BC)

Moniezia sp. (Alta)

Taenia hydatigena cysticercus (Alta, Ont, Sask)

Taenia krabbei cysticercus (Alta, NWT, Ont, Que, Sask, YT)

Taenia ovis / T. krabbei cysticercus (Alta, BC)

Taenia sp. metacestode (Ont)

Thysanosoma actinioides (Alta)

NEMATODA

Dictyocaulus hadweni (Alta, Nfld)

D. viviparus (Alta, BC, Que)

Elaphostrongylus cervi (Ont)

Nematodirella alcidis (Alta)

N. longispiculata (Alta, BC, Nfld)

Nematodirus sp. (Ont, Que)

Onchocerca cervipedis (Alta, BC)

Orthostrongylus macrotis (Alta)

Ostertagia sp. (Alta)

Parelaphostrongylus odocoilei (Alta)

$P$. tenuis (Man, NB, NS, Ont)

$P$. tenuis larva (Ont)

Protostrongylus macrotis (Alta)

Setaria labiatopapillosa (Alta)

S. yehi (Alta)

Setaria sp. (Que)

Trichostrongylus longispicularis (Alta)

Trichuris sp. (Alta, BC)

Alces a americana (Clinton)

NEMATODA

Parelaphostrongylus tenuis (Ont) 
Alces $a$. andersoni Peterson

PROTOZOA

Sarcocystis sp. cyst (Alta)

CESTOIDEA

Echinococcus granulosus hydatid cyst (Alta)

Moniezia sp. (Alta)

Taenia hydatigena cysticercus (Alta)

Taenia krabbei cysticercus (Alta)

Thysanosoma actinioides (Alta)

NEMATODA

Dictyocaulus viviparus (Alta)

Nematodirella longispiculata (Alta)

Onchocerca cervipedis (Alta)

Ostertagia sp. (Alta)

Parelaphostrongylus odocoilei (Alta)

Setaria sp. (Alta)

Trichuris sp. (Alta)

Cervus elaphus L.

Wapiti

PROTOZOA

Eucoccidiida gen. sp. (Sask)

Sarcocystis sp. cysts (Alta)

TREMATODA

Fascioloides magna (Alta, BC, Ont, Que, Sask)

CESTOIDEA

Cysticercus tenuicollis (Alta, BC, Ont)

Echinococcus granulosus hydatid cyst (Alta,

BC, NWT, Ont, Sask, YT)

Moniezia benedini (Alta)

Taenia hydatigena cysticercus (Alta)

Thysanosoma actinioides (Alta, BC)

NEMATODA

Cooperia oncophora (Alta)

Dictyocaulus viviparus (Alta, BC)

Nematodirella alcidis (Alta)

$N$. longispiculata (Alta)

Nematodirus helvetianus (Alta)

Orthostrongylus macrotis (Alta)

Ostertagia sp. (Alta)

Parelaphostrongylus tenuis (Ont)

Setaria cervi (Alta)

Trichostrongylus axei (Alta)

Trichuris ovis (Alta)

Trichuris sp. (Alta)

Odocoileus hemionus (Rafinesque) Mule deer PROTOZOA

Sarcocystis sp. cysts (Alta)

CESTOIDEA

Cysticercus tenuicollis (BC, NWT)

NEMATODA

Dictyocaulus viviparus (Alta)

Nematodirus odocoilei (BC)

Parelaphostrongylus odocoilei (Alta)

Parelaphostrongylus sp. larva (Alta)

Setaria yehi (BC)

Trichuris sp. (Alta)
Odocoileus hemionus columbianus (Richardson)

Syn.: Odocoileus columbianus Merriam

TREMATODA

Fasciola hepatica (BC)

Fascioloides magna (Alta, BC, Can)

CESTOIDEA

Cysticercus ovis $(\mathrm{BC})$

C. tenuicollis (BC)

Cysticercus sp. cyst (Alta, BC)

Echinococcus granulosus hydatid cysts (Alta, $\mathrm{BC})$

Moniezia benedini (Alta, BC)

Taenia krabbei / $T$. ovis cysticercus (BC)

Thysanosoma actinioides (Alta, BC)

NEMATODA

Capillaria sp. (BC)

Dictyocaulus viviparus (BC)

Elaeophora schneideri (BC)

Haemonchus contortus (BC)

Nematodirella longispiculata (BC)

$N$. l. antilocaprae (BC)

Nematodirus filicollis $(\mathrm{BC})$

Onchocerca cervipedis (BC)

Oesophagostomum venulosum (BC)

Ostertagia circumcincta $(\mathrm{BC})$

Parelaphostrongylus odocoilei (Alta, BC)

Setaria cervi (BC)

Trichostrongylus sp. (BC)

Trichuris ovis $(\mathrm{BC})$

Odocoileus hemionus hemionus (Rafinesque)

TREMATODA

Fascioloides magna (Alta, BC)

CESTOIDEA

Cysticercus tenuicollis (Alta, $\mathrm{BC}$ )

Echinococcus granulosus hydatid cyst (Alta)

Moniezia benedini (Alta, BC)

Thysanosoma actinioides (BC)

NEMATODA

Dictyocaulus viviparus (Alta, BC)

Onchocerca cervipedis (BC)

Parelaphostrongylus odocoilei (Alta)

$P$. tenuis (Ont)

Varestrongylus alpenae (Sask)

Odocoileus virginianus (Zimmermann)

\section{PROTOZOA}

White-tailed deer

Sarcocystis sp. cysts (Alta, Ont)

TREMATODA

Fascioloides magna (Alta, BC, Ont, Que)

CESTOIDEA

Echinococcus granulosus hydatid cyst (Alta,

NWT, Ont, Sask, YT)

Taenia hydatigena cysticercus (Ont)

NEMATODA

Dictyocaulus viviparus (Ont) 
Nematodirus odocoilei (Que)

Oesophagostomum venulosum (Ont)

Onchocerca cervipedis (Ont)

Ostertagia dikmansi (Ont)

O. mossi (Ont)

O. odocoilei (Ont)

Parelaphostrongylus andersoni (BC)

$P$. tenuis (Man, NB, NS, Ont, Que, Sask)

$P$. tenuis larva (Ont)

Setaria tundra (Ont)

Spiculopteragia bohmi (Que)

Varestrongylus alpenae (Sask)

Odocoileus virginianus borealis Miller

NEMATODA

Elaphostrongylus odocoilei (Ont)

Parelaphostrongylus tenuis (Ont)

Odocoileus virginianus dacotensis Goldman and Kellogg

NEMATODA

Parelaphostrongylus tenuis larva (Alta)

Odocoileus virginianus ochrourus Bailey

CESTOIDEA

Cysticercus tenuicollis (BC)

Odocoileus sp.

CESTOIDEA

Taenia hydatigena cysticercus (Alta, BC, Ont, Que)

Rangifer tarandus (L.) Caribou PROTOZOA

Besnoitia sp. (NWT)

Sarcocystis sp. cyst (NWT)

CESTOIDEA

Avitellina arctica (NWT)

Cysticercus tarandi (NWT)

C. tenuicollis (Alta, BC, NWT)

Cysticercus sp. cyst (Alta, BC)

Echinococcus granulosus hydatid cyst (Alta,

NWT, Ont, Sask, YT)

Moniezia expansa (Sask)

Moniezia sp. (NWT)

Taenia hydatigena cysticercus (NWT)

T. krabbei cysticercus (Alta, NWT, Sask)

$T$. ovis / T. krabbei cysticercus (Alta)

NEMATODA

Dictyocaulus viviparus (NWT)

Elaphostrongylus cervi (Ont)

Nematodirella longispiculata (NWT)

Ostertagia oreamni (Alta)

$O$. trifurcata (NWT)

Ostertagia spp. (Alta)

Setaria cervi (Sask)

S. yehi (NWT)
Rangifer tarandus caribou (Gmelin)

PROTOZOA

Amoebae (BC)

Besnoitia sp. (Sask)

Sarcocystis sp. cyst (Sask)

TREMATODA

Fascioloides magna (Que)

CESTOIDEA

Moniezia sp. (BC)

NEMATODA

Dictyocaulus sp. (BC)

Elaphostrongylus cervi (Nfld)

E. cervi larva (Man, Nfld)

Nematodirella longispiculata (BC)

Nematodirus sp. (BC)

Onchocerca sp.(BC)

Ostertagia circumcincta $(\mathrm{BC})$

O. trifurcata $(\mathrm{BC})$

Parelaphostrongylus tenuis larva (NS)

Protostrongylus larva (Man, Ont)

Rangifer tarandus groenlandicus (L.)

CESTOIDEA

Echinococcus granulosus hydatid cyst (Man)

Taenia hydatigena cysticercus (not specified)

T. krabbei cysticercus (NWT, Ont, Sask, YT)

$T$. ovis cysticercus (NWT)

NEMATODA

Dictyocaulus viviparus (NWT)

Elaphostrongylus cervi larva (Man, Nfld)

Rangifer tarandus tarandus $\mathrm{L}$.

NEMATODA

Parelaphostrongylus tenuis (Ont)

Rangifer tarandus terraenovae (Gmelin)

CESTOIDEA

Cysticercus tenuicollis (Nfld)

NEMATODA

Parelaphostrongylus tenuis (Ont)

Rangifer sp.

NEMATODA

Skrjabinema oreamni (Alta)

CERVIDAE OF UNDETERMINED SPECIES

Barren-ground caribou

PROTOZOA

Besnoitia sp. (NWT)

Deer

TREMATODA

Fascioloides magna (Ont)

CESTOIDEA

Cysticercus tenuicollis (Ont) 
Echinococcus granulosus hydatid cyst (Ont) Taenia hydatigena cysticercus (Alta)

Elk

TREMATODA

Fascioloides magna (Ont)

Moose

PROTOZOA

Sarcocystis sp. cyst (not specified)

TREMATODA

Fascioloides magna (Ont)

CESTOIDEA

Cysticercus tarandi (Ont)

C. tenuicollis (Ont)

Echinococcus granulosus hydatid cyst (Man,

Ont)

Taenia hydatigena cysticercus (Man)

T. krabbei cysticercus (Man)

NEMATODA

Dictyocaulus sp. (Man)

Setaria sp. (Man)

Reindeer

CESTOIDEA

Echinococcus granulosus hydatid cyst (Alta, NWT, Ont, Sask, YT)

\section{ORDER CARNIVORA}

\section{FAMILY CANIDAE}

Alopex lagopus (L.)

Arctic fox

PROTOZOA

Coccidia (NWT)

Eucoccidiida gen. sp. (NWT)

CESTOIDEA

Echinococcus multilocularis (NWT)

Taenia crassiceps (NWT)

T. polyacantha (NWT)

NEMATODA

Toxascaris leonina (NWT)

Trichinella spiralis cyst (NWT)

Canis latrans Say

Coyote

PROTOZOA

Toxoplasma gondii (Ont)

TREMATODA

Alaria americana (Alta, Ont)

A. arisaemoides (Alta, Man, Ont)

A. marcianae (Man)

Metorchis conjunctus (Alta)

Paragonimus kellicotti (Ont)

CESTOIDEA

Diphyllobothrium sp. (Alta)

Echinococcus granulosus (Alta, Man, Ont)

E. multilocularis (Man)
Mesocestoides kirbyi (Alta)

Mesocestoides sp. (Ont)

Taenia hydatigena (Alta, Man, Ont)

T. krabbei (Alta)

T. laticollis (Ont)

T. multiceps (Alta)

$T$. pisiformis (Alta, Man, Ont)

T. twitchelli (Alta)

Taenia sp.(Man)

NEMATODA

Ancylostoma caninum (Alta)

Capillaria aerophila (Alta)

C. hepatica (Sask)

C. plica (Ont)

Capillaria sp. (not specified)

Dioctophyma renale (Alta)

Oslerus osleri (Alta, Man, Ont)

Physaloptera sp. (not specified)

Toxocara canis (Alta)

Toxascaris leonina (Alta, Man)

Trichinella spiralis larva (BC, Que)

Canis lupus L.

TREMATODA

Alaria americana (Alta, Ont, YT)

A. arisaemoides (Man, NWT, YT)

A. canis (Ont)

A. marcianae (Man, Que)

Metorchis conjunctus (Alta, Sask)

CESTOIDEA

Diphyllobothrium sp. (Alta, NWT, Ont)

Echinococcus granulosus (Alta, Man, NWT,

Ont, Que, YT)

Mesocestoides kirbyi (Alta, YT)

Taenia crassiceps (Man, Ont)

T. hydatigena (Alta, Man, NWT, Ont, Que,

YT)

T. krabbei (Alta, NWT, Ont, YT)

T. laticollis (Ont)

T. multiceps (Alta)

T. omissa (Alta)

T. pisiformis (Alta, NWT, Ont)

T. serialis (NWT, YT)

T. taeniaeformis (Alta)

Taenia sp. (Man, NWT, Ont, Que, YT)

NEMATODA

Capillaria aerophila (Alta)

Dioctophyma renale (Que)

Oslerus osleri (Man, Ont)

Spirocerca arctica (NWT)

S. lupi (NWT)

Toxocara canis (Alta)

Toxascaris leonina (Alta, Man, NWT, YT)

Trichinella spiralis larva (Alta, NWT, Que, YT)

Trichinella sp. (Alta) 
Vulpes vulpes (L.)

Syn.: Vulpes fulva De Kay

Red fox

PROTOZOA

Toxoplasma gondii (Ont)

TREMATODA

Alaria alata (Man, PEI, Que)

A. americana (NB, NS, Ont, PEI, Que)

A. arisaemoides (Man, NB, NS, Ont)

A. marcianae (Sask)

A. mustelae (NB, NS, Ont)

Cryptocotyle lingua (NB, NS)

Echinostoma revolutum (NB, NS)

Metorchus conjunctus (NB, NS)

Paragonimus kellicotti (Ont)

Phagicola longa (Ont, Que)

CESTOIDEA

Diphyllobothrium cordatum (Ont)

D. latum (Man, Ont)

Diphyllobothrium sp. (Ont)

Dipylidium caninum (Que)

Echinococcus multilocularis (Man, Sask)

Mesocestoides sp. (Ont)

Taenia crassiceps (Man, NB, NS, Ont)

$T$. pisiformis (Ont)

Taenia sp.(Man, Ont)

NEMATODA

Ancylostoma caninum (Que)

Capillaria aerophila (NB, NS, Ont)

C. plica (Ont)

Crenosoma decoratum (Ont)

C. vulpis (NB, Nfld, NS, Ont)

Toxascaris leonina (Man, Ont, Que)

Toxocara canis (Man, NB, NS, Ont)

Trichinella spiralis cyst/larva (Que)

Trichuris vulpis (Que)

\section{CANIDAE OF UNDETERMINED SPECIES}

Coyote x Dog

\section{CESTOIDEA}

Taenia crassiceps (Ont)

$T$. pisiformis (Ont)

NEMATODA

Oslerus osleri (Ont)

Red fox

\section{CESTOIDEA}

Taenia crassiceps (Ont)

NEMATODA

Crenosoma sp. (Ont)

\section{FAMILY FELIDAE}

Felis concolor L.

Mountain lion

CESTOIDEA

Taenia hydatigena (Alta)

T. omissa (Alta, BC)

\section{NEMATODA}

Trichinella spiralis larva (BC)

Trichinella sp. larva (Alta)

Felis concolor vancouverensis Nelson and Goldman CESTOIDEA

Taenia hydatigena $(\mathrm{BC})$

T. taeniaeformis $(\mathrm{BC})$

Lynx canadensis Kerr

Syn.: Lynx lynx (L.)

TREMATODA

Alaria americana (Ont)

A. canis (Ont)

Alaria sp. (Alta, NWT)

CESTOIDEA

Multiceps sp. (Alta, NWT)

Taenia laticollis (Alta, BC, Nfld, NWT)

T. macrocystis (Nfld)

$T$. pisiformis (Alta, NWT)

$T$. rileyi (Alta, BC, NWT)

Taenia sp. (Alta, NWT)

NEMATODA

Cylicospirura subaequalis (Alta, Nfld, NWT)

Physaloptera praeputialis (Alta)

Physaloptera sp. (Ont)

Toxocara cati (Alta, NWT, Ont)

Toxascaris leonina (Alta, Nfld, NWT)

Trichinella spiralis larva (BC)

Troglostrongylus wilsoni (Alta, NWT)

Lynx rufus (Schreber)

Bobcat

TREMATODA

Alaria americana (Ont)

A. canis (Ont)

CESTOIDEA

Taenia laticollis $(\mathrm{BC})$

T. macrocystis (NB, NS)

T. rileyi $(\mathrm{BC})$

NEMATODA

Trichinella spiralis cyst (BC, Que)

Lynx rufus fasciatus Rafinesque

CESTOIDEA

Taenia taeniaeformis (BC)

NEMATODA

Toxascaris leonina (BC)

FAMILY MUSTELIDAE

Gulo gulo (L.)

Wolverine

TREMATODA

Alaria sp. (NWT)

CESTOIDEA

Diphyllobothrium sp. (NWT)

Taenia martis (NWT)

T. twitchelli (NWT)

Lynx 
NEMATODA

Baylisascaris devosi (NWT)

Physaloptera sp. (NWT)

Trichinella spiralis cyst (BC, Man)

Lutra canadensis Schreber

River otter

TREMATODA

Alaria americana mesocercaria (Ont)

CESTOIDEA

Schistocephalus solidus plerocercoid (Nfld)

NEMATODA

Dracunculus lutrae (Ont)

Dracunculus sp. (Ont)

Skrjabingylus lutrae (Ont)

ACANTHOCEPHALA

Echinorhynchus lateralis (Nfld)

Lutra canadensis canadensis (Schreber)

NEMATODA

Filaroides canadensis (Ont)

Gnathostoma miyazakii (Ont)

Martes americana (Turton)

Marten

TREMATODA

Alaria taxideae (Man, NWT)

CESTOIDEA

Mesocestoides sp. (Ont)

Taenia martis (NWT, Ont)

T. mustelae (Man, NWT)

Taenia sp. (Man, NWT)

NEMATODA

Baylisascaris devosi (Man)

Capillaria aerophila (Ont)

C. putorii (Ont)

Trichinella spiralis cyst (BC, Man, Que)

Trichinella sp. larva (Man)

Martes americana actuosa (Osgood)

TREMATODA

Alaria taxideae (NWT)

CESTOIDEA

Taenia martis (NWT)

T. mustelae (NWT)

Martes americana americana (Turton)

NEMATODA

Baylisascaris devosi (Ont)

Martes martes L.

Pine marten

PROTOZOA

Toxoplasma gondii (Ont)

Martes pennanti (Erxleben)

PROTOZOA

Toxoplasma gondii (Ont)

TREMATODA

Alaria americana (Ont)
A. mustelae (Man)

Alaria sp. (Ont)

Echinostoma sp. (Ont)

Metorchis conjunctus (Man)

CESTOIDEA

Echinococcus granulosus (Ont)

Taenia sibirica (Man)

NEMATODA

Baylisascaris devosi (Man)

Capillaria plica (Ont)

C. putorii (Ont)

Crenosoma petrowi (Ont)

Dracunculus insignis (Ont)

Molineus sp. (Man)

Physaloptera sp. (Man)

Sobolevingylus sp. (Ont)

Trichinella spiralis (Man)

Trilobostrongylus bioccai (Ont)

Martes pennanti pennanti (Erxleben)

NEMATODA

Baylisascaris devosi (Ont)

Trilobostrongylus bioccai (Ont)

Mephitis mephitis (Schreber) Striped skunk

PROTOZOA

Toxoplasma gondii (Alta, Ont, Sask)

TREMATODA

Alaria canadensis (Que)

Apophallus sp. (Que)

Euryhelmis pyriformis (Que)

Fibricola cratera (Que)

Paragonimus kellicotti (Ont)

CESTOIDEA

Mesocestoides latus (Que)

Oochoristica oklahomensis (Que)

NEMATODA

Andersonstrongylus captivensis (Ont)

Arthrocephalus lotoris (Que)

Baylisascaris columnaris (Ont, Que)

B. devosi (Ont)

Capillaria plica (Ont)

C. procyonis (Ont)

C. putorii (Ont)

Crenosoma mephitidis (Ont, Que)

Dipetalonema mephitis (Ont, Que)

D. mephitis microfilaria (Ont, Que)

Dracunculus insignis (Ont)

Filaroides martis (Que)

F. mephitis (Ont)

Physaloptera maxillaris (Ont, Que)

Physaloptera sp. (Que)

Placoconus lotoris (Que)

Skrjabingylus chitwoodorum (Ont)

S. magnus (Que)

Skrjabingylus sp. (Que)

Trichinella spiralis larva (BC) 
Mustela erminea L.

CESTOIDEA

Taenia mustelae (Nfld, Ont)

\section{NEMATODA}

Aelurostrongylus pridhami (Nfld)

Capillaria putorii (Ont)

Capillaria sp. larva (Nfld)

Dracunculus sp. (Ont)

Skrjabingylus nasicola (BC, Nfld)

Mustela erminea anguinae Hall

NEMATODA

Skrjabingylus nasicola $(\mathrm{BC})$

Mustela erminea richardsonii Bonaparte

NEMATODA

Skrjabingylus nasicola (Man)

Mustela frenata Lichtenstein Long-tailed weasel NEMATODA

Trichinella spiralis larva (BC)

Mustela frenata longicauda Bonaparte

NEMATODA

Skrjabingylus nasicola (Man)

Mustela putorius (L.)

Ferret (Lab)

PROTOZOA

Eimeria mustelae (Ont)

TREMATODA

Alaria americana mesocercaria (Ont)

CESTOIDEA

Taenia mustelae (Ont)

T. mustelae metacestode (Ont)

NEMATODA

Baylisascaris devosi (Ont)

Mustela vison Schreber

Mink

PROTOZOA

Eimeria mustelae (Ont)

Hepatozoon sp. (Ont)

Toxoplasma gondii (Ont)

TREMATODA

Alaria craterum (Ont)

A. mustelae (Ont)

Euparyphium inerme (Ont)

E. melis (Ont)

Hemistomum craterum (Ont)

Nanophyetus salmincola (Ont)

Paragonimus kellicotti (Ont)

Paragonimus sp. (Ont)

Parametorchis canadensis (Ont)

Plagiorchis proximus (Ont)

CESTOIDEA

Mesocestoides sp. (Ont)
Taenia mustelae (Nfld, Ont)

$T$. mustelae metacestode (Ont)

Taenia sp. (Ont)

NEMATODA

Aelurostrongylus pridhami (Nfld, Ont)

Ascaris sp. (Ont)

Capillaria mustelorum (Nfld)

C. putorii (Ont)

Capillaria sp. (Ont)

Dioctophyma renale (Man, Ont, Que)

Dracunculus insignis (Ont)

Dracunculus sp. (Ont)

Filaria sp. (Ont)

Filaroides bronchialis (Ont)

F. martis (Ont)

Oslerus sp. (Ont)

Skrjabingylus nasicola (Ont)

Strongyloides sp. (Ont)

Trichinella spiralis larva (BC)

Mustela vison vison Schreber

NEMATODA

Aelurostrongylus pridhami (Ont)

Crenosoma hermani (Ont)

Filaroides martis (Ont)

Spilogale putorius gracilis MerriamSpotted skunk

Syn.: Spilogale gracilis olympica Merriam NEMATODA

Skrjabingylus chitwoodorum (BC)

Taxidea taxus (Schreber) Badger

PROTOZOA

Sarcocystis campestris (Sask)

NEMATODA

Ascaris sp. (Alta)

Crenosoma petrowi (Ont)

Dracunculus sp. (Man)

Physaloptera sp. (Alta)

MUSTELIDAE OF LNDETERMINED SPECIES

Ferret

TREMATODA

Alaria americana (Ont)

A. arisaemoides mesocercaria (Ont)

Mink

TREMATODA

Dicrocoelium dendriticum (PEI)

Paragonimus kellicotti (Ont)

Paragonimus sp. (Can)

NEMATODA

Dioctophyma renale (Ont)

Dracunculus insignis (Ont)

Filaroides martis (Ont) 
Otter

NEMATODA

Dracunculus insignis (Ont)

\section{FAMILY PROCYONIDAE}

Procyon lotor (L.)

Raccoon

PROTOZOA

Toxoplasma gondii (Ont)

TREMATODA

Apophallus venustus (Can, Ont)

Fibricola laruei (Que)

Pharyngostomoides adenocephala (Ont)

$P$. procyonis (Ont)

NEMATODA

Ascaris sp. (Ont)

Capillaria plica (Ont)

C. procyonis (Ont)

C. putorii (Ont)

Capillaria sp. (Ont)

Crenosoma goblei (Ont)

Dracunculus insignis (Ont)

Dracunculus sp. (Ont)

Physaloptera sp. (Ont, Que)

Placoconus lotoris (Que)

Trichinella spiralis cyst/larva (Que)

T. s. var. pseudospiralis (Man)

Trichinella sp. (Man)

Procyon lotor hirtus Nelson and Goldman

TREMATODA

Alaria marcianae (Sask)

Microphallus sp. (Sask)

Pharyngostomoides adenocephala (Sask)

Plagiorchus elegans (Sask)

CESTOIDEA

Atriotaenia procyonis (Sask)

NEMATODA

Physaloptera sp. (Sask)

\section{PROCYONIDAE OF UNDETERMINED SPECIES}

Raccoon

NEMATODA

Dracunculus insignis (Ont)

\section{FAMILY URSIDAE}

Ursus americanus Pallas

Black bear

PROTOZOA

Eucoccidiida gen. sp.(Que)

Eimeria albertensis (Alta)

E. borealis (Alta)

Toxoplasma gondii (Ont)

TREMATODA

Alaria americana (Ont)
CESTOIDEA

Diphyllobothrium ursi (Que)

D. ursi eggs (Que)

Diphyllobothrium sp. egg (Que)

Taenia hydatigena (Alta, Que)

T. krabbei (Alta, Ont, Que)

Taeniid ova (Que)

NEMATODA

Ascarid gen. sp. egg (Que)

Ascaris sp. (Que)

Baylisascaris transfuga (Alta, Ont, Que)

B. transfuga egg (Que)

Crenosoma petrowi (Ont)

C. potos (Ont)

C. vulpis (Ont)

Crenosoma sp. (Ont)

Dirofilaria ursi (Alta, Ont, Que)

D. ursi microfilaria (Ont)

D. ursi ova (Que)

Physaloptera rara(Ont)

Trichinella spiralis cyst/larva (BC, Que)

Trichinella sp.larva (Ont)

Uncinaria yukonensis (Que, YT)

Ursus americanus americanus Pallus

NEMATODA

Dirofilaria ursi (Ont, Que)

Ursus americanus vancouveri Hall

NEMATODA

Toxascaris transfuga $(\mathrm{BC})$

Ursus arctos $\mathrm{L}$.

CESTOIDEA

Diphyllobothrium ursi (BC, YT)

Taenia krabbei (NWT, YT)

NEMATODA

Baylisascaris transfuga (BC, NWT?, YT?)

Dirofilaria ursi (BC, YT)

Trichinella spiralis larva (BC, NWT, YT)

Trichinella sp. larva (Alta)

Uncinaria yukonensis (NWT, YT)

Ursus maritimus Phipps

Polar bear

NEMATODA

Trichinella spiralis (Man)

$T$. spiralis cyst (Man, Nfld)

\section{ORDER CHIROPTERA}

FAMILY VESPERTILIONIDAE

Eptesicus fuscus

Big brown bat

(Palisot de Beauvois)

PROTOZOA

Trypanosoma hedricki (Ont)

TREMATODA 
Acanthatrium eptesici (BC)

Plagiorchis vespertilionis $(\mathrm{BC})$

Plagiorchis sp. (BC)

Prosthodendrium macnabi $(\mathrm{BC})$

Prosthodendrium sp. (BC)

CESTOIDEA

Hymenolepis gertschi $(\mathrm{BC})$

NEMATODA

Litomosoides guiterasi (BC)

Rictularia lucifugus (BC)

Lasionycteris noctivagans

Silver-haired bat

(Le Conte)

TREMATODA

Acanthatrium nycteridis (BC)

Plagiorchis vespertilionis (BC)

Plagiorchis sp. (BC)

Prosthodendrium macnabi $(\mathrm{BC})$

Prosthodendrium sp. (BC)

Lasiurus cinereus (Palisot de Beauvois)Hoary bat TREMATODA

Limatulum gastroides $(\mathrm{BC})$

Plagiorchis vespertilionis (BC)

Prosthodendrium silvai (BC)

Prosthodendrium sp. (BC)

CESTOIDEA

Cestoidea gen. sp. (BC)

Myotis californicus

California bat

(Audubon and Bachman)

TREMATODA

Plagiorchis vespertilionis (BC)

Myotis lucifugus (Le Conte) Little brown bat

PROTOZOA

Trypanosoma myoti (Ont)

TREMATODA

Lecithodendrium anticum (not specified)

L. posticum (not specified)

Plagiorchis vespertilionis (BC)

Prosthodendrium swansoni (BC)

Prosthodendrium sp. (BC)

CESTOIDEA

Hymenolepis sp. (BC)

NEMATODA

Capillaria eubursata (BC)

Rictularia lucifugus (BC)

Rictularia sp. (BC)

Myotis volans longicrus (True) Long-legged bat

TREMATODA

Plagiorchis vespertilionis (BC)

Prosthodendrium naviculum (BC)

NEMATODA

Allintoshius nycticeius (BC)

Rictularia lucifugus (BC)
Myotis yumanensis saturatus Miller Yuma bat

Syn.: $\quad$ Myotis saturatus Miller

PROTOZOA

Trypanosoma evotomys (BC)

\section{ORDER INSECTIVORA}

\section{FAMILY SORICIDAE}

Blarina brevicauda (Say) Short-tailed shrew

PROTOZOA

Toxoplasma gondii (Ont)

Trypanosoma soricis (Ont)

NEMATODA

Metastrongyloidea gen. sp. larva (Ont)

Sorex cinereus Kerr

Masked shrew

NEMATODA

Capillaria plica (Que)

Sorex fumeus Miller

Smoky shrew

NEMATODA

Metastrongyloidea gen. sp. larva (Ont)

Sorex vagrans Baird

Vagrant shrew

PROTOZOA

Trypanosoma soricis (BC)

NEMATODA

Trichinella spiralis larva (BC)

\section{ORDER LAGOMORPHA}

\section{FAMILY LEPORIDAE}

Lepus americanus Erxleben Snowshoe rabbit

PROTOZOA

Eimeria athabascensis (Alta)

E. holmesi (Alta)

E. keithi (Alta)

E. leporis (Alta)

E. robertsoni (Alta)

E. rochesterensis (Alta)

E. rowani (Alta)

E. ruficaudati (Alta)

E. townsendi (Alta)

Eimeria sp. (Man)

CESTOIDEA

Cittotaenia pectinata americana (Man)

Taenia macrocystis larva (NB, NS)

$T$. pisiformis cysticercus (Alta, Man, Nfld)

$T$. serialis coenurus (Alta, Man, Nfld)

NEMATODA

Dirofilaria scapiceps (Alta, NWT, Ont)

Nematoda gen. sp. (Nfld)

Nematodirus triangularis (Man, Nfld)

Obeliscoides cuniculi (Alta)

O. c. multistriatus (Alta, Ont) 
Passalurus nonanulatus (Man)

Protostrongylus boughtoni (Alta, Man, NB,

Ont, Que)

Strongyloides papillosus (Man)

Synthetocaulus leporis (Man)

Trichostrongylus sp. (Man)

Trichuris leporis (Man)

ACANTHOCEPHALA

Acanthocephala gen. sp. (Man)

Lepus americanus americanus Erxleben

NEMATODA

Protostrongylus boughtoni (Alta)

Lepus americanus pallidus Cowan

\section{CESTOIDEA}

Taenia serialis cysts (BC)

Lepus americanus struthopus Bangs

TREMATODA

Dicrocoelium dendriticum (Nfld)

CESTOIDEA

Cittotaenia pectinata (Nfld)

Multiceps sp. cyst (Nfld)

Taenia pisiformis cysticercus (Nfld)

$T$. taeniaformis cysticercus (Nfld)

NEMATODA

Dirofilaria scapiceps (NB)

Obeliscoides cuniculi (Nfld)

Lepus capensis L.

Cape hare

NEMATODA

Dirofilaria scapiceps (Ont)

Obeliscoides cuniculi cuniculi (Ont)

Oryctolagus cuniculus (L.)

Lab rabbit

Syn.: Lepus cuniculus L.

TREMATODA

Fascioloides magna (Alta, BC)

NEMATODA

Dirofilaria scapiceps (Ont)

Obeliscoides cuniculi cuniculi (Ont)

O. c. multistriatus (Ont)

Protostrongylus boughtoni (Alta)

Sylvilagus floridanus (Allen) Eastern cottontail PROTOZOA

Toxoplasma gondii (Ont)

NEMATODA

Dirofilaria scapiceps (Ont)

Obeliscoides cuniculi cuniculi (Ont)

Sylvilagus nuttallii (Bachman) Cottontail

Syn.: Lepus sylvaticus Allen

PROTOZOA

Trypanosoma leporis-sylvaticus (Alta)
FAMILY OCHOTONIDAE

Ochotona collaris (Nelson)

Collared pika

PROTOZOA

Eimeria banffensis (Alta, YT)

E. barretti (Alta, YT)

E. calentinei (Alta, YT)

E. circumborealis (Alta, YT)

E. klondikensis (Alta, YT)

E. princepsis (Alta, YT)

Isospora marquardti (Alta, YT)

I. yukonensis (YT)

CESTOIDEA

Schizorchis caballeroi (YT)

NEMATODA

Cephaluris alaskensis (Alta, YT)

Cephaluris coloradensis (Alta, YT)

Labiostomum rauschi (YT)

L. talkeetnaeuris (YT)

Ochotona princeps (Richardson)

PROTOZOA

Eimeria barretti (Alta, YT)

E. calentinei (Alta, YT)

E. circumborealis (Alta, YT)

E. klondikensis (Alta, YT)

E. princepsis (Alta, YT)

E. worleyi (Alta)

Eucoccidiida gen. sp. (Alta)

Isospora marquardti (Alta, YT)

CESTOIDEA

Schizorchis caballeroi (Alta)

S. ochotonae (Alta)

NEMATODA

Cephaluris alaskensis (Alta, YT)

Graphidiella ochotonae (Alta)

Labiostomum coloradensis (Alta)

Murielus harpespiculus (Alta)

Physaloptera bispiculata (Alta)

\section{LAGOMORPHA OF UNDETERMINED SPECIES}

Cottontail

CESTOIDEA

Cysticercus pisiformis (Ont)

Lab rabbit

TREMATODA

Diplostomum spathaceum (Ont)

CESTOIDEA

Cysticercus pisiformis (Ont)

Rabbit

PROTOZOA

Eucoccidiida gen. sp. (Ont)

CESTOIDEA

Cysticercus pisiformis (Ont) 
Unidentified Leporidae

PROTOZOA

Trypanosoma evotomys (BC)

Varying hare

CESTOIDEA

Cysticercus pisiformis (Ont)

Multiceps serialis (Ont)

\section{ORDER RODENTIA}

\section{FAMILY CASTORIDAE}

Castor canadensis Kuhl

Beaver

TREMATODA

Echinostoma revolutum (NS)

Stephanoproraoides lawi (NS, Ont)

Stichorchis subtriquetrus (Alta, Nfld, NS, Ont, Que)

NEMATODA

Castorstrongylus castoris (Alta, NB, Nfld)

Dipetalonema sprenti (Ont)

D. sprenti microfilaria (Ont)

Travassosius americanus (Alta, NB, Nfld, NS)

ACANTHOCEPHALA

Polymorphus paradoxus (Alta)

Castor canadensis leucodontus Gray

TREMATODA

Stichorchis subtriquetrum (BC)

FAMILY CAVIIDAE

Cavia porcellus $\mathrm{L}$.

Guinea pig

TREMATODA

Fascioloides magna (Alta, BC)

NEMATODA

Anisakis sp. (Que)

Parelaphostrongylus tenuis (Ont)

FAMILY ERETHIZONTIDAE

Erethizon dorsatum (L.)

TREMATODA

Porcupine

Schistosomatium douthitti (Ont)

S. douthitti ova (Ont)

\section{FAMILY GEOMYIDAE}

Thomomys talpoides (Richardson)Northern pocket

CESTOIDEA gopher

Paranoplocephala variabilis (Alta)

Taenia mustelae metacestode(Alta)

NEMATODA

Capillaria hepatica (Alta)

Litomosoides carinii (Alta)
Protospirura ascaroidea (Alta)

Trichuris fossor (Alta)

FAMILY MURIDAE

Clethrionomys gapperi (Vigors) Gapper's red-

TREMATODA

Plagiorchis sp. (Lab, Que)

CESTOIDEA

Andrya communis (Alta)

A. macrocephala (Alta, Nfld, Que)

Andrya sp. (Nfld, Que)

Catenotaenia dendritica (Alta, Nfld, Que)

Cestoidea gen. sp. (Nfld, Que)

Cladotaenia globifera metacestode (Ont)

C. mirsoevi (Alta)

Hymenolepis horrida (Alta, Nfld, Que)

Paruterina rauschi metacestode (Ont)

Taenia mustelae metacestode (Alta, Ont, Que)

NEMATODA

Capillaria hepatica (Man, Ont)

Heligmosomum carolinensis (Nfld, Que)

Protospirura muris (Alta, Nfld, Ont)

Clethrionomys g. gapperi (Vigors)

NEMATODA

Capillaria hepatica (Ont)

Clethrionomys gapperi saturatus (Rhoads)

PROTOZOA

Trypanosoma evotomys (BC)

Clethrionomys sp.

CESTOIDEA

Cladotaenia mirsoevi (Alta)

Dicrostonyx groenlandicus Collared lemming (Traill)

NEMATODA

Pelodera strongyloides larva (NWT)

Dicrostonyx groenlandicus richardsoni Merriam CESTOIDEA

Andrya arctica (Man)

Taenia crassiceps cysticercus (Man)

Dicrostonyx hudsonius (Pallas) Labrador collared CESTOIDEA lemming

Paranoplocephala variabilis (Nfld, Que)

NEMATODA

Heligmosomum hudsonius (Nfld, Que)

Dicrostonyx torquatus (Pallas) Collared lemming CESTOIDEA

Andrya arctica (NWT)

Taenia crassiceps cysticercus (NWT) 
Lemmus sibiricus Kerr

Brown lemming

Syn.: Lemmus trimucronatus (Richardson)

NEMATODA

Pelodera strongyloides larva (NWT)

Lemmus sibiricus trimucronatus (Richardson)

Syn.: Lemmus trimucronatus trimucronatus

(Richardson)

CESTODA

Paranoplocephala lemmi (NWT)

Meriones unguiculatus

Gerbil

(Milne-Edwards, 1867)

CESTOIDEA

Echinococcus multilocularis hydatid cyst(Que)

E. multilocularis sibiricensis (Que)

Taenia crassiceps cysticercus (Man)

Mesocricetus auratus

Golden hamster

(Waterhouse, 1840)

PROTOZOA

Giardia mesocricetus (Ont)

G. microti (Ont)

G. muris (Ont)

NEMATODA

Trichinella spiralis (Man)

Microtus chrotorrhinus (Miller)

Rock vole

CESTOIDEA

Andrya bairdi (Nfld, Que)

A. macrocephala (Alta, Nfld, Que)

Hymenolepis horrida (Que)

NEMATODA

Heligmosomoides polygyrus (Nfld, Que)

Microtus ochrogaster (Wagner) Prairie vole

Syn.: Pedomys ochrogaster (Wagner)

CESTOIDEA

Andrya macrocephala (Alta)

Taenia mustelae metacestode (Alta)

Microtus pennsylvanicus (Ord) Meadow vole

PROTOZOA

Giardia microti (Ont)

Trypanosoma microti (Ont)

TREMATODA

Plagiorchis muris (Nfld, Que)

Quinqueserialis quinqueserialis (Nfld, Que)

Schistosomatium douthitti (Man)

CESTOIDEA

Andrya communis (Alta)

A. macrocephala (Alta, Nfld, Que)

Cladotaenia globifera metacestode (Man)

Echinococcus multilocularis hydatid cyst

(Sask)

Hymenolepis horrida (Alta)

Paranoplocephala infrequens (Alta, Nfld, Que)
$P$. variabilis (Alta, Nfld, Que)

Paruterina candelabraria plerocercoid (Man)

Taenia crassiceps cysticercus (Ont)

T. mustelae metacestode (Alta, Nfld, Ont, Que)

NEMATODA

Capillaria hepatica (Man)

Pelodera strongyloides larva (NWT)

Rictularia coloradensis (Alta)

Syphacia obvelata (Nfld, Que)

ACANTHOCEPHALA

Polymorphus paradoxus (Alta)

Microtus pennsylvanicus drummondii (Audubon and Bachman)

CESTOIDEA

Hymenolepis johnsoni (NWT)

Taenia crassiceps cysticercus (Man)

Taenia sp. metacestode (Man)

NEMATODA

Syphacia obvelata (Man)

Microtus p. pennsylvanicus (Ord)

NEMATODA

Capillaria hepatica (Ont)

Microtus p. terraenovae (Bangs)

TREMATODA

Brachylaima sp. (Nfld)

Microtus sp. lab.

PROTOZOA

Trypanosoma microti (Ont)

Mus musculus L.

House mouse

PROTOZOA

Toxoplasma gondii (Ont)

TREMATODA

Alaria americana mesocercaria (Ont)

A. arisaemoides mesocercaria (Ont)

CESTOIDEA

Choanotaenia nebraskensis (Alta)

Taenia taeniaeformis cysticercus (Alta)

NEMATODA

Pelodera strongyloides larva (NWT)

Syphacia obvelata (Nfld)

Mus musculus (laboratory)

House mouse

CESTOIDEA

Echinococcus granulosus (Lebanon Strain)

(Que)

E. multilocularis sibiricensis (Que)

Taenia crassiceps cysticercus (Man)

Taenia mustelae metacestode (Ont)

NEMATODA

Capillaria hepatica (Ont)

Trichinella spiralis (Man, Que)

T. spiralis larva (Man, NWT, Que) 
$T$. spiralis cyst (Que)

T. s. var. pseudospiralis (Man)

Trichuris muris (Ont)

Neotoma cinerea (Ord) Bushy-tailed wood rat CESTOIDEA

Andrya neotomae (Alta)

Ondatra zibethicus (L.)

Muskrat

PROTOZOA

Eimeria stiedae (Ont)

Encephalitozoon cuniculi (Sask)

Frenkelia microti (Ont)

Protozoa gen. sp. (Man)

TREMATODA

Alaria craterum (Ont)

A. mustelae (Man, Ont)

Catatropus filamentis (Ont)

Echinochasmus schwartzi (Ont)

Echinoparyphium contiguum (BC, Ont)

Echinoparyphium sp. (NB)

Echinostoma callawayensis (Ont)

E. revolutum (BC, Man, NB, Ont, Que)

Echinostomum sp. (Alta)

Metorchis conjunctus (Ont)

Notocotylus filamentis (Man)

$N$. quinqueserialis (Ont)

$N$. urbanensis (BC, NB, Ont)

Nudacotyle novicia (NB, Ont)

Opisthorchis tonkae (NB)

Plagiorchis noblei (Man)

$P$. proximus (BC, NB, Ont)

Psilostomum ondatrae (Ont)

Quinqueserialis quinqueserialis (BC, Man,

NB, Ont)

Q. zibethicai (Que)

Schistosomatium douthitti (NB)

S. douthitti ova (Man)

S. douthitti immature (Man)

Wardius zibethicus (Man, NB, Ont)

CESTOIDEA

Andrya macrocephala (NB)

Cladotaenia cysticercus (BC)

Cysticercus fasciolaris (BC, Ont)

C. talpae (Ont)

Echinococcus multilocularis sibiricensis (Que)

Hymenolepis evaginata (Alta, BC, NB, Ont)

H. octocoronata (BC)

Hymenolepis sp. (Alta, Man)

Taenia crassiceps cysticercus (Ont)

T. mustelae metacestode (BC, NB, Ont)

T. taeniaeformis cysticercus (Man, Ont)

Taenia sp. (Ont)

NEMATODA

Capillaria hepatica (Alta, BC, Man, Ont)

C. michiganensis (Man, Ont)

C. ransomia $(\mathrm{BC})$
Dracunculus insignis (Ont)

Dracunculus sp. (Ont)

Eustrongylides sp. larva (Ont)

Heligmosomum carolinensis (Man)

Trichuris opaca (BC, Ont)

ACANTHOCEPHALA

Polymorphus paradoxus (Alta)

Polymorphus sp. (BC, Man)

Ondatra zibethicus obscurus (Bangs)

TREMATODA

Diplostomum mergi (Nfld)

Echinoparyphium contiguum (Nfld)

$E$. recurvatum (Nfld)

Echinostoma revolutum (Nfld)

Paramphistomatidae gen. sp. (Nfld)

Plagiorchis proximus (Nfld)

Quinqueserialis quinqueserialis (Nfld)

CESTOIDEA

Anoplocephalid (Nfld)

Hymenolepis evaginata (Nfld)

NEMATODA

Capillaria michiganensis (Nfld)

Trichostrongylus calcaratus (Nfld)

Ondatra zibethicus osoyoosensis (Lord)

CESTOIDEA

Taenia taeniaeformis cysticercus (BC)

Peromyscus leucopus

White-footed mouse

(Rafinesque)

CESTOIDEA

Taenia mustelae metacestode (NWT)

NEMATODA

Capillaria hepatica (Ont)

Peromyscus leucopus noveboracensis (Fischer)

NEMATODA

Capillaria hepatica (Ont)

Peromyscus maniculatus (Wagner) Deer mouse

PROTOZOA

Eimeria arizonensis (BC)

E. siniffi $(\mathrm{BC})$

Giardia peromysci(Ont)

Toxoplasma gondii (Ont)

Trypanosoma peromysci (Alta)

TREMATODA

Alaria americana mesocercaria (Ont)

A. arisaemoides mesocercaria (Ont)

Digenea gen. sp. metacercaria (Que)

Postharmostomum helicis (Que)

CESTOIDEA

Choanotaenia peromysci (Alta)

Cladotaenia globifera metacestode (Ont)

Echinococcus multilocularis hydatid cyst

(Alta, Man) 
Paruterina candelabraria plerocercoid (Man)

$P$. rauschi (Ont)

Taenia crassiceps cysticercus (Ont)

T. mustelae metacestode (Nfld, Ont, Que)

NEMATODA

Capillaria hepatica (Alta, Man, Ont)

Capillaria sp. (Que)

Metastrongyloidea gen. sp. larva (Ont)

Pelodera strongyloides larva (NWT)

Protospirura muris (Que)

Syphacia peromysci (Que)

Trichinella spiralis larva (BC)

Peromyscus maniculatus gracilis (Le Conte)

CESTOIDEA

Hymenolepis bennetti(Ont)

Peromyscus maniculatus nebrascensis (Coues)

PROTOZOA

Trypanosoma peromysci (Alta)

Phenacomys intermedius Merriam Heather vole CESTOIDEA

Andrya communis (Alta)

Paranoplocephala variabilis (Alta)

NEMATODA

Heligmosomoides polygyrus (Alta)

Rattus norvegicus (Berkenhout) . Norway rat

PROTOZOA

Chilomastix bettencourti (Que)

Eimeria carinii (Que)

E. miyairii (Que)

E. separata (Que)

Entamoeba muris (Que)

Giardia muris (Ont, Que)

G. simoni (Ont)

Toxoplasma gondii (Ont)

Trichomonas muris (Que)

CESTOIDEA

Cysticercus fasciolaris (Que)

Hymenolepis diminuta (Que)

H. murina (Que)

Hymenolepididae gen. sp. (Nfld)

Taenia taeniaeformis cysticercus (Nfld)

NEMATODA

Capillaria hepatica (Que)

Heterakis spumosa (Nfld, Que)

Nippostrongylus muris (Que)

Protospirura muris (Que)

Strongyloides ratti (Que)

Trichosomoides crassicauda (Que)

Rattus sp.

PROTOZOA

Wild rat

Trypanosoma lewisi(Ont)

NEMATODA

Trichinella spiralis (NB, NS, PEI)
T. spiralis larva (BC, NB, NS, $\mathrm{PEI})$

Sigmodon hispidus Say and Ord

Cotton rat

TREMATODA

Metorchis conjunctus (Que)

CESTOIDEA

Echinococcus granulosus (Lebanon strain)

(Que)

E. multilocularis hydatid cyst (Que)

E. m. sibiricensis (Que)

Taenia crassiceps cysticercus (Man)

Synaptomys borealis Northern bog lemming

(Richardson)

CESTOIDEA

Andrya arctica (Alta)

Paranoplocephala infrequens (Nfld, Que)

Taenia mustelae metacestode (Nfld, Que)

Synaptomys cooperi cooperi Baird

NEMATODA

Capillaria hepatica (Ont)

MURIDAE OF UNDETERMINED SPECIES

Field mice

PROTOZOA

Giardia microti (NS)

Hamster (Lab)

PROTOZOA

Giardia microti (Ont)

G. muris (Ont)

TREMATODA

Diplostomum spathaceum (Ont)

Mouse (Lab)

PROTOZOA

Giardia muris (NS, Ont)

TREMATODA

Alaria americana mesocercaria (Ont)

A. arisaemoides mesocercaria (Ont)

Diplostomum spathaceum (Ont)

CESTOIDEA

Echinococcus granulosus canadensis hydatid cyst (Que)

E. multilocularis hydatid cyst (Que)

E.m. sibiricensis (Que)

Taenia crassiceps cysticercus (Man, Ont)

NEMATODA

Capillaria hepatica (Ont)

Trichinella sp. (Man)

Muskrat

NEMATODA

Dracunculus insignis (Ont)

Rat (Lab) 
PROTOZOA

Giardia mesocricetus (Ont)

G. muris (NS)

G. $\operatorname{simoni}($ Ont)

CESTOIDEA

Echinococcus multilocularis sibiricensis

hydatid cyst (Que)

NEMATODA

Trichinella spiralis (Man)

$T$. spiralis cyst/larva $(\mathrm{BC})$

\section{FAMILY SCIURIDAE}

Eutamias amoenus (J. A. Allen) NEMATODA

Yellow-pine

Syphacia eutamii (Alta)

chipmunk

Eutamias minimus (Bachman) Least chipmunk

PROTOZOA

Trypanosoma tamiasi (Ont)

CESTOIDEA

Hymenolepis sp. (Sask)

Taenia mustelae metacestode (Ont, Sask)

NEMATODA

Citellinema bifurcatum (Sask)

Heteroxynema cucullatum (Sask)

Glaucomys sabrinus (Shaw)

PROTOZOA

Northern flying

Eimeria dorneyi (Ont)

squirrel

TREMATODA

Plagiorchis proximus (Sask)

CESTOIDEA

Andrya sciuri (Sask)

Cladotaenia globifera metacestode (Sask)

Marmota monax (L.)

Woodchuck

CESTOIDEA

Taenia crassiceps cysticercus (Ont)

T. mustelae metacestode (Ont)

NEMATODA

Ackertia marmotae (Ont)

Obeliscoides cuniculi cuniculi(Ont)

Marmota monax canadensis (Erxleben)

NEMATODA

Ackertia marmotae (Ont, Que)

Marmota vancouverensis SwarthVancouver Island CESTOIDEA marmot

Diandrya vancouverensis (BC)

NEMATODA

Baylisascaris laevis (BC)

Sciurus carolinensis Gmelin

PROTOZOA

Eimeria confusa (Ont)
E. ontarioensis (Ont)

Eimeria sp. (Ont)

CESTOIDEA

Taenia crassiceps cysticercus (Ont)

Spermophilus columbianus Columbian ground

(Ord)

PROTOZOA squirrel

Eimeria bilamellata (Alta)

E. callospermophili (Alta)

E. lateralis (Alta)

Trypanosoma otospermophili (Alta, YT)

NEMATODA

Trichinella spiralis larva (BC)

Spermophilus franklinii Franklin's ground (Sabine)

PROTOZOA

Eimeria bilamellata (Alta)

squirrel

E. callospermophili (Alta)

E. spermophili (Alta)

Trypanosoma otospermophili (Alta, YT)

TREMATODA

Alaria mustelae mesocercaria (Sask)

Plagiorchis proximus (Sask)

CESTOIDEA

Choanotaenia spermophili (Sask)

Hymenolepis citelli (Man, Sask)

Taenia mustelae metacestode (Sask)

$T$. taxidiensis cysticercus (Sask)

NEMATODA

Capillaria hepatica (Sask)

Citellinema bifurcatum (Sask)

Physaloptera spinicauda (Man, Sask)

P. spinicauda larva (Sask)

Rictularia citelli (Man)

Spirura infundibuliformes (Sask)

$S$. infundibuliformes larva (Sask)

Spermophilus parryii (Richardson) Arctic ground

Syn.: Spermophilus undulatus plesius

Osgood squirrel

PROTOZOA

Trypanosoma otospermophili (Alta, YT)

Spermophilus richardsonii

(Sabine)

Richardson's

PROTOZOA

Eimeria bilamellata (Alta)

E. callospermophili (Alta)

E. lateralis (Alta)

E. spermophili (Alta)

Sarcocystis campestris (Sask)

Trypanosoma citelli (Alta)

T. otospermophili (Alta, YT)

TREMATODA

Plagiorchis proximus (Man, Sask)

CESTOIDEA 
Choanotaenia spermophili (Sask)

Hymenolepis citelli (Man, Sask)

H. diminuta (Alta)

NEMATODA

Baylisascaris laevis (Sask)

Baylisascaris sp. larva (Sask)

Capillaria hepatica (Sask)

Citellinema bifurcatum (Man, Sask)

Physaloptera spinicauda (Sask)

P. spinicauda larva (Sask)

Spirura infundibuliformis (Man, Sask)

S. infundibuliformis larva (Sask)

Strongyloides robustus (Sask)

Syphacia citelli (Sask)

Trichostrongylus axei (Sask)

Trichuris leporis (Man)

Trichuris sp. (Man, Sask)

ACANTHOCEPHALA

Mediorhynchus robustus (Sask)

Spermophilus tridecemlineatus Thirteen-lined (Mitchell)

\section{PROTOZOA}

ground squirrel

Trypanosoma otospermophili (Alta, YT)

CESTOIDEA

Cladotaenia circi cysticeroid (Sask)

Hymenolepis citelli (Man, Sask)

Taenia mustelae metacestode (Sask)

NEMATODA

Citellinema bifurcatum (Sask)

Oxynema sp. (Alta)

Physaloptera spinicauda (Alta, Man, Sask)

P. spinicauda larva (Sask)

Rictularia citelli (Man)

Spirura infundibuliformis (Man, Sask)

S. infundibuliformis larvae (Sask)

Strongyloides robustus (Sask)

ACANTHOCEPHALA

Moniliformis spiradentatis (Man)

Tamias striatus (L.) $\quad$ Eastern chipmunk PROTOZOA

Trypanosoma tamiasi (Ont)

TREMATODA

Scaphiostomum pancreaticum (Ont)

CESTOIDEA

Taenia crassiceps cysticercus (Ont)

T. mustelae metacestode (Ont)

NEMATODA

Metastrongyloidea gen. sp.larva (Ont)

Tamiasciurus hudsonicus (Erxleben) Red squirrel

PROTOZOA

Eimeria tamiasciuri (Alta)

E. toddi (Alta)

Hepatozoon sp. (Alta)

TREMATODA
Plagiorchis proximus (Sask)

CESTOIDEA

Andrya primordialis (Alta, Sask)

Cladoiaenia sp. cysticercus (Alta)

Hymenolepis horrida (Alta)

Paruterina candelabraria plerocercoid (Alta, Sask)

Taenia crassiceps cysticercus (Ont)

T. mustelae metacestode (Alta, Ont)

T. rileyi cysticercus (Alta)

NEMATODA

Ascaris sp. (Alta)

Capillaria hepatica (Ont)

Citellinema bifurcatum (Alta, Sask)

Physaloptera sp. (Alta)

Strongyloides robustus (Sask)

Syphacia thompsoni (Alta, Sask)

Trichinella spiralis larva (BC)

Tamiasciurus hudsonicus hudsonicus (Erxleben) NEMATODA

Citellinema bifurcatum (Man)

\section{SCIURIDAE OF UNDETERMINED SPECIES}

Squirrel

PROTOZOA

Trypanosoma sp. (BC)

FAMILY ZAPODIDAE

Napaeozapus insignis insignis

(Miller)

Syn.: Napaeozapus insignis algonquinensis

Woodland jumping

Prince mouse

CESTOIDEA

Hymenolepis bennetti (Ont)

NEMATODA

Capillaria hepatica (Ont)

Zapus hudsonius

Meadow jumping mouse

(Zimmermann)

TREMATODA

Plagiorchis proximus (Nfld, Que)

Quinqueserialis quinqueserialis (Nfld, Que)

CESTOIDEA

Choanotaenia sp. (Alta)

Mesocestoides sp. (Nfld, Que)

Taenia mustelae metacestode (Ont)

NEMATODA

Protospirura muris (Nfld, Que) 


\section{REFERENCES}

ADAMS, J.R. 1964. Taeniids from lynx in British Columbia with a comment on Taenia monostephanos von Linstow and other taeniids with a single crown of hooks. Abstract of paper given at the 1st International Congress for Parasitology, Rome, September 1964.

ADDISON, E.M. 1973. Life cycle of Dipetalonema sprenti Anderson (Nematoda: Filarioidea) of beaver ( Castor canadensis). Canadian Journal of Zoology 51: 403-416.

- 1978. Crenosoma spp. (Nematoda:

Metastrongyloidea) from black bears, Ursus americanus, of central Ontario. Canadian Journal of Zoology 56: 1993-1997.

1980. Transmission of Dirofilaria ursi Yamaguti, 1941 (Nematoda:

Onchocercidae) of black bears (Ursus americanus) by blackflies (Simuliidae). Canadian Journal of Zoology 58: 1913-1922.

ADDISON, E.M., AND B. BOLES. 1978.

Helminth parasites of wolverine, Gulo gulo, from the District of Mackenzie, Northwest Territories. Canadian Journal of Zoology 56: 2241-2242.

ADDISON, E.M., A. FYVIE, AND F.J. JOHNSON. 1979. Metacestodes of moose, Alces alces, of the Chapleau Crown Game Preserve, Ontario. Canadian Journal of Zoology 57: 1619-1623.

ADDISON, E.M., M.J. PYBUS, AND H.J. RIETVELD. 1978. Helminths and arthropod parasites of black bear, Ursus americanus, in central Ontario. Canadian Journal of Zoology 56: 2122-2126.

AMIN, O.M. 1982. Acanthocephala. pp. 933-940. In Synopsis and Classification of Living Organisms. McGraw-Hill Book Co., New York.

ANDERSON, R.C. 1952. Description and relationships of Dirofilaria ursi Yamaguti, 1941, and a review of the genus Dirofilaria Railliet and Henry, 1911. Transactions of the Royal Canadian Institute 29: 35-64.

1953. Dipetalonema sprenti n. sp. from

Castor canadensis Kuhl. Parasitology 43:

215-221.

1956. Elaphostrongylus odocoilei

Hobmaier and Hobmaier, 1934 in the

cranial case of Odocoileus virginianus borealis Miller. Canadian Journal of Zoology 34: 167-173.

1962a. The systematics and transmission of new and previously described metastrongyles (Nematoda:

Metastrongylidae) from Mustela vison.

Canadian Journal of Zoology 40: 893-920.

1962b. The helminth and arthropod parasites of the white-tailed deer (Odocoileus virginianus): a general review. Transactions of the Royal Canadian Institute, 34: 57-92.

1963a. The incidence, development, and experimental transmission of

Pneumostrongylus tenuis Dougherty

(Metastrongyloidea: Protostrongylidae) of the meninges of the white-tailed deer (Odocoileus virginianus borealis) in Ontario. Canadian Journal of Zoology 41: 775-792.

- 1963b. Further studies on the taxonomy of metastrongyles (Nematoda:

Metastrongyloidea) of Mustelidae in Ontario. Canadian Journal of Zoology 41: 801-809.

1964a. Gnathostoma miyazakii n. sp. from the otter (Lutrac. canadensis) with comments on $G$. sociale (Leidy, 1858) of mink ( Mustela vison). Canadian Journal of Zoology 42: 249-254.

- 1964b. Neurologic disease in moose infected experimentally with

Pneumostrongylus tenuis from white-tailed deer. Pathologia Veterinaria 1: 289-322. -1965a. An examination of wild moose exhibiting neurologic signs, in Ontario. Canadian Journal of Zoology 43: 635-639. 1971b. Neurologic disease in reindeer ( Rangifer tarandus tarandus) introduced into Ontario. Canadian Journal of Zoology 49: 159-166.

ANDERSON, R.C., M.W. LANKESTER, AND U.R. STRELIVE. 1966. Further experimental studies of Pneumostrongylus tenuis in cervids. Canadian Journal of Zoology 44: 851-861.

ANDERSON, R.C., AND U.R. STRELIVE. 1966. The transmission of Pneumostrongylus tenuis to guinea pigs. Canadian Journal of Zoology 44: 533-540.

1967. The penetration of

Pneumostrongylus tenuis into the tissues of white-tailed deer. Canadian Journal of Zoology 45: 285-289.

- 1968. The experimental transmission of Pneumostrongylus tenuis to caribou 
( Rangifer tarandus terraenovae). Canadian Journal of Zoology 46: 503-510.

ANONYMOUS 1930. Experimental fur farm. Ont. Dept. Game Fish. Annu. Rep. 23(1929): 9-12.

\section{A note on Parametorchis} canadensis (Law) from Ontario mink. Report of the Ontario Veterinary College for 1930: 39 .

\section{Survey to ascertain the} geographical distribution of the large American liver fluke ( Fascioloides magna) in Ontario. Report of the Ontario Veterinary College for 1949: 35.

BAIRD, W. 1853. Catalogue of the species of Entozoa or intestinal worms contained in the collection of the British Museum, London. Woodfall and Kinder, London. $132 \mathrm{pp}$.

BAKER, M.R., AND R.C. ANDERSON. 1975.

Seasonal changes in abomasal worms (Ostertagia spp.) in white-tailed deer (Odocoileus virginianus) at Long Point, Ontario. Canadian Journal of Zoology 53: 87-96.

BAKER, M.R., AND S.R. PURSGLOVE, JR. 1976. Oesophagostomum Molin 1861 in white-tailed deer (Odocoileus virginianus) of North America. Journal of Parasitology 62: 166-168.

BALASINGAM, E. 1964a. Studies on the life cycle and developmental morphology of Placoconus lotoris (Schwartz, 1925)

Webster, 1956 (Ancylostomidae: Nematoda). Canadian Journal of Zoology 42: 869-902.

1964b. On the pathology of Placoconus lotoris infections in raccoons ( Procyon lotor). Canadian Journal of Zoology 42: 903905.

BALLANTYNE, R.J., AND W.M. SAMUEL. 1984. Diagnostic morphology of the thirdstage larvae of the three species of Parelaphostrongylus (Nematoda, Metastrongyloidea). Journal of Parasitology 70: 602-604.

BANFIELD, A.W.F. 1954. Preliminary investigations of the barren ground caribou. Part II. Life history, ecology, and utilization. Wildlife Management Bulletin (Ottawa) Series 1 No. 10B.

BARKER, I.K. 1970. The penetration of oncospheres of Taenia pisiformis into the intestine of the rabbit. Canadian Journal of Zoology 48: 1329-1332.

BARON, R.W. 1970. The occurrence of
Echinococcus multilocularis Leuckart, 1863 and of other helminths in the red fox, Vulpes vulpes, in southern Manitoba. Canadian Journal of Zoology 48: 1132.

BARON, R.W. 1971. The occurrence of Paruterina candelabraria (Goeze, 1782) and Cladotaenia globifera (Batsch, 1786) in Manitoba. Canadian Journal of Zoology 49: 1399-1400.

BARON, R.W., M.E. RAU, AND C.E. TANNER. 1974. Growth of secondary Echinococcus multilocularis in experimentally infected hosts. Canadian Journal of Zoology 52: 587-589.

BARRETT, M.W. 1972. A review of the diet, condition, diseases, and parasites of the Cypress Hills moose. 8th North American Moose Conference Workshop, Thunder Bay, Ontario. Ontario Ministry of National Resources, Toronto, 60-79.

BARRETT, M.W., AND G.A. CHALMERS. 1972. Capillaria hepatica (Nematoda:

Trichuridae) in pronghorn antelope (Antilocapra americana Ord) in Alberta. Journal of Wildlife Diseases 8: 332-334.

BARTLETT, C.M. 1983a. Zoogeography and taxonomy of Dirofilaria scapiceps (Leidy, 1886 ) and $D$. uniformis Price, 1957 (Nematoda: Filarioidea) of lagomorphs in North America. Canadian Journal of Zoology 61: 1011-1022.

BARTLETT, C.M. 1984a. Development of Dirofilaria scapiceps (Leidy, 1886) (Nematoda: Filarioidea) in lagomorphs. Canadian Journal of Zoology 62: 965-979.

BARTLETT, C.M. 1984b. Pathology and epizootiology of Dirofilaria scapiceps (Leidy, 1886) (Nematoda: Filarioidea) in Sylvilagus floridanus (Allen) and Lepus americanus Erxleben. Journal of Wildlife Diseases 20: 197-206.

BECKLUND, W.W. 1965. Nematodirus maculosus sp. n. (Nematoda:

Trichostrongylidae) from the mountain goat, Oreamnos americanus, in North America. Journal of Parasitology 51: 945947.

BECKLUND, W.W., AND M.L. WALKER. 1967. Nematodirus odocoilei sp.n. (Nematoda: Trichostrongylidae) from the black-tailed deer, Odocoileus hemionus, in North America. Journal of Parasitology 53: 392394.

1968. Ostertagia dikmansi sp.n. (Nematoda: Trichostrongylidae) from deer, Odocoileus virginianus, with a key to the species of medium stomach worms of 
Odocoileus in North America. Journal of Parasitology 54: 44l-444.

1969. Taxonomy, hosts, and geographic distribution of the Setaria (Nematoda:

Filarioidea) in the United States and Canada. Journal of Parasitology 55: 359368.

BENSON, D.A. 1958. Moose "sickness" in Nova Scotia-1. Canadian Journal of Comparative Medicine 22: 244-248.

BEVERLEY-BURTON, M., AND P.R. SWEENY. 1972. Intranuclear, paracrystalline inclusions in various cells of Quinqueserialis quinqueserialis and Notocotylus urbanensis (Trematoda: Notocotylidae). Canadian Journal of Zoology 50: 345-348.

BINDERNAGEL, J.A., AND R.C. ANDERSON. 1972a. Newer aspects of the problem of meningeal worm ( Pneumostrongylus tenuis) in North America: Distribution in white-tailed deer and moose. 8th North American Moose Conference Workshop, Thunder Bay, Ontario 1-17. 1972b. Distribution of the meningeal worm in white-tailed deer in Canada. Journal of Wildlife Management 36: 13491353.

BLOOD, D.A. 1963. Parasites from California bighorn sheep in southern British Columbia. Canadian Journal of Zoology 41: 913-918.

BOUGHTON, R.V. 1932. The influence of helminth parasitism on the abundance of the snowshoe rabbit in western Canada. Canadian Journal of Research Section D, Zoological Sciences 7: 524-547.

BOURQUE, M. 1981. The masked shrew ( Sorex cinereus), a new host for Capillaria plica. Canadian Journal of Zoology 59: 23932394.

1985. A survey of Trichinella spiralis in wild carnivores in southwestern Quebec. The Canadian Veterinary Journal 26: 203204.

BOWER, S.M., AND P.T.K. WOO. 1981. Two new species of trypanosomes (subgenus Schizotrypanum) in bats from southern Ontario. Canadian Journal of Zoology 59: 530-545.

BROOKS, D.R., R.T. O'GRADY, AND D.R. GLEN. 1985. Phylogenetic analysis of the Digenea (Platyhelminthes: Cercomeria) with comments on their adaptive radiation. Canadian Journal of Zoology 63: 411-443. BURSEY, C.C., AND M.D.B. BURT. 1970.
Taenia macrocystis (Diesing, 1850), its occurrence in eastern Canada and Maine, U.S.A., and its life cycle in wild felines (Lynx rufus and L. canadensis) and hares (Lepus americanus). Canadian Journal of Zoology 48: 1287-1293.

BUSH, A.O., AND W.M. SAMUEL. 1978. The genus Travassosius Khalil, 1922

(Nematoda: Trichostrongyloidea) in beaver, Castor spp.: a review and suggestion for speciation. Canadian Journal of Zoology 56: 1471-1474.

1981. A review of helminth communities in beaver ( Castor spp.) with a survey of Castor canadensis in Alberta, Canada. In: First Worldwide Furbearer Conf. J.A. Chapman and D. Pursley eds. pp. 678-689.

BUTTERWORTH, E.W., AND M. BEVERLEYBURTON. 1980. The taxonomy of Capillaria spp. (Nematoda: Trichuroidea) in Carnivorous mammals from Ontario, Canada. Systematic Parasitology 1: 211236.

1981. Observations on the prevalence and intensity of Capillaria spp. (Nematoda:

Trichuroidea) in wild Carnivora from Ontario, Canada. Proceedings of the Helminthological Society of Washington 48 : 24-27.

BUTTERWORTH, E.W., AND J.C. HOLMES. 1984. Character divergence in two species of trematodes (Pharyngostomoides: Strigeoidea). Journal of Parasitology 70: 315-316.

CAMERON, A.E. 1923. Notes on buffalo: anatomy, pathological conditions, and parasites. Veterinary Journal 79: 331-336.

CAMERON, T.W.M. 1936. Studies on the heterophyid trematode, Apophallus venustus (Ransom, 1920) in Canada. Part I. Morphology and taxonomy. Canadian Journal of Research Section D, Zoological Sciences 14: 59-69.

-1937a. Studies on the heterophyid trematode Apophallus venustus (Ransom, 1920) in Canada. Part II. Life history and bionomics. Canadian Journal of Research Section D, Zoological Sciences 15: 38-51. - 1937b. Studies on the heterophyid trematode Apophallus venustus (Ransom, 1920) in Canada. Part III. Further hosts. Canadian Journal of Research Section D, Zoological Sciences 15: 275.

1937c. Cited in: The distribution of

Paragonimus. Journal of Parasitology 23: 382-388.

1940. Metorchis conjunctus (Cobbold, 
1859) Looss, 1899. Report on the

Proceedings of the III International

Congress of Microbiology (Abstract): 437-

438. New York.

1960. Trichinosis in Canada. Wiadomosci

Parazytologiczne 6: 304.

CAWTHORN, R.J., AND R.C. ANDERSON.

1976a. Development of Physaloptera

maxillaris (Nematoda: Physalopteroidea)

in skunk (Mephitis mephitis) and the role of paratenic and other hosts in its life cycle.

Canadian Journal of Zoology 54: 313-323.

1976b. Seasonal population changes of

Physaloptera maxillaris (Nematoda:

Physalopteroidea) in striped skunk

(Mephitis mephitis). Canadian Journal of

Zoology 54: 522-525.

CAWTHORN, R.J., G.A. WOBESER, AND A.A.

GAJADHAR. 1983. Description of

Sarcocystis campestris sp. n. (Protozoa:

Sarcocystidae): a parasite of the badger

Taxidea taxus with experimental

transmission to the Richardson's ground squirrel, Spermophilus richardsonii.

Canadian Journal of Zoology 61: 370-377.

CHADEE, K., AND T.A. DICK. 1982.

Designation and freezing resistance of isolates of Trichinella spiralis from wild carnivores. Journal of Wildlife Diseases 18: 169-173.

CHALMERS, G.A., AND M.W. BARRETT. 1974.

Echinococcus multilocularis Leuckart, 1863

in rodents in southern Alberta. Canadian

Journal of Zoology 52: 1091.

CHAPIN, E.A. 1925. New nematodes from North

American mammals. Journal of

Agricultural Research 30: 677-681.

CHOQUETTE, L.P.E. 1951. Helminthes parasites trouves chez la mouffette Mephitis mephitis dans la Province de Quebec.

Canadian Journal of Comparative Medicine 15: $62-64$.

1952. Dirofilaria desportesi sp. nov., a

filariid from the black bear in Canada.

Canadian Journal of Zoology 30: 344-351.

CHOQUETTE, L.P.E., E. BROUGHTON, AND

G.G. GIBSON. 1973. Schistosomatium

douthitti (Cort, 1914) Price, 1929 in a

porcupine (Erethizon dorsatum) in eastern

Ontario, Canada. Canadian Journal of

Zoology 51: 1317.

CHOQUETTE, L.P.E., E. BROUGHTON, F.L.

MILLER, H.C. GIBBS, and J.G.

COUSINEAU. 1967. Besnoitiosis in

barren-ground caribou in northern Canada.

Canadian Veterinary Journal 8: 282-287.

CHOQUETTE, L.P.E., G.G. GIBSON, E. KUYT,
AND A.M. PEARSON. 1973. Helminths of wolves, Canis lupus L., in the Yukon and Northwest Territories. Canadian Journal of Zoology 51: 1087-1091.

CHOQUETTE, L.P.E., G.G. GIBSON, AND A.M. PEARSON. 1969. Helminths of the grizzly bear, Ursus arctos L. in northern Canada. Canadian Journal of Zoology 47: 167-170.

CHOQUETTE, L.P.E., G.G. GIBSON, AND B. SIMARD. 1971. Fascioloides magna (Bassi, 1875) Ward, 1917 (Trematoda) in woodland caribou, Rangifer tarandus caribou (Gmelin), of northeastern Quebec, and its distribution in wild ungulates in Canada. Canadian Journal of Zoology 49: 280-281.

CHOQUETTE, L.P.E., A.H. MACPHERSON, AND J.G. COUSINEAU. 1962. Note on the occurrence of Echinococcus multilocularis Leuckart, 1863 in the arctic fox in Canada. Canadian Journal of Zoology 40: 1167.

CHOQUETTE, L.P.E., AND D.H. PIMLOTT. 1956. Gastrointestinal parasites of beaver in Newfoundland. Canadian Journal of Zoology 34: 209.

CHOQUETTE, L.P.E., L.K. WHITTEN, G. RANKIN, AND C.M. SEAL. 1957. Note on parasites found in reindeer ( $R$ angifer tarandus) in Canada. Canadian Journal of Comparative Medicine and Veterinary Science 21: 199-203.

CLIFF, G.M., R.C. ANDERSON, AND F.F. MALLORY. 1978. Dauerlarvae of Pelodera strongyloides (Schneider, 1860) (Nematoda: Rhabditidae) in the conjunctival sacs of lemmings. Canadian Journal of Zoology 56 2117-2121.

CONKLIN, R.L., AND A.D. BAKER. 1930.

Presence of the lancet fluke, Dicrocoelium dendriticum (Rudolphi, 1819), in Canada. Journal of Parasitology 17: 18-19.

CONNELL, R., AND A.H. CORNER. 1957. Polymorphus paradoxus sp. nov. (Acanthocephala) parasitizing beavers and muskrats in Alberta, Canada. Canadian Journal of Zoology 35: 525-533.

COWAN, I. MCT. 1942. Report upon some diseases and parasites of game birds and game and fur-bearing mammals in British Columbia. Report of the Provincial Game Commission, British Columbia (1941), K40K45.

1944. Parasites, diseases, and injuries of game animals in Banff, Jasper, and Kootenay National Parks. Lands, Parks, and Forests Branch, National Parks Bureau pp. 1-42.

1946. Parasites, diseases, injuries, and 
anomalies of the Columbian black-tailed deer, Odocoileus hemionus columbianus (Richardson), in British Columbia. Canadian Journal of Research Section D, Zoological Sciences 24: 71-103.

- 1948. The occurrence of the granular tapeworm Echinococcus granulosus in wild game in North America. Journal of Wildlife Management 12: 105-106.

1951. The diseases and parasites of big game mammals of western Canada.

Proceedings of the Fifth Annual Game Convention, Vancouver, B.C., 1951. 5: 3764.

CRAIG, R.E., AND R.C. ANDERSON. 1972. The genus Crenosoma (Nematoda:

Metastrongyloidea) in New World mammals. Canadian Journal of Zoology 50: 1555-1561.

CRAIG, R.E., AND R.A. BORECKY. 1976.

Metastrongyles (Nematoda:

Metastrongyloidea) of fisher ( Martes pennanti) from Ontario. Canadian Journal of Zoology 54: 806-807.

CRICHTON, V.F.J. 1971. Dracunculiasis in otter, mink and raccoon from Ontario. Journal of Parasitology 57(section 2, part 1): 13.

1979. An experimental moose hunt on Hecla Island, Manitoba. Proceedings of the North American Moose Conference Workshop 15: 245-279.

1980. Manitoba's second experimental moose hunt on Hecla Island. Proceedings of the North American Moose Conference Workshop 16: 489-526.

CRICHTON, V.F.J., AND M. BEVERLEYBURTON. 1973. Dracunculus lutrae n. sp. (Nematoda: Dracunculoidea) from the otter, Lutra canadensis, in Ontario, Canada. Canadian Journal of Zoology 51: 521-529. 1974. Distribution and prevalence of Dracunculus spp. (Nematoda: Dracunculoidea) in mammals in Ontario. Canadian Journal of Zoology 52: 163-167. 1975. Migration, growth, and morphogenesis of Dracunculus insignis (Nematoda: Dracunculoidea). Canadian Journal of Zoology 53: 105-113.

- 1977. Observations on the seasonal prevalence, pathology and transmission of Dracunculus insignis (Nematoda:

Dracunculoidea) in the raccoon (Procyon lotor (L.)) in Ontario. Journal of Wildlife Diseases 13: 273-280.

CRICHTON, V.J., AND R.E. URBAN. 1970.
Dioctophyme renale (Goeze, 1782)

(Nematoda: Dioctophymata) in Manitoba mink. Canadian Journal of Zoology 48: 591-592.

DAUPHINE, JR., T.C. 1975. The disappearance of caribou reintroduced to Cape Breton Highlands National Park. Canadian FieldNaturalist 89: 299-310.

DE VOS, A., AND A.E. ALLIN. 1949. Some notes on moose parasites. Journal of Mammalogy 30: $430-431$.

DICK, T.A. 1983. Infectivity of isolates of Trichinella and the ability of an arctic isolate to survive freezing temperatures in the raccoon, Procyon lotor, under experimental conditions. Journal of Wildlife Diseases 19: 333-336.

DICK, T.A., AND M. BELOSEVIC. 1978. Observations on a Trichinella spiralis isolate from a polar bear. Journal of Parasitology 64: 1143-1145.

DICK, T.A., AND K. CHADEE. 1983. Interbreeding and gene flow in the genus Trichinella. Journal of Parasitology 69: 176-180.

DICK, T.A., AND R.D. LEONARD. 1979. Helminth parasites of fisher Martes pennanti (Erxleben) from Manitoba, Canada. Journal of Wildlife Diseases 15: 409-412.

DIES, K.H. 1979. Helminths recovered from black bears in the Peace River region of northwestern Alberta. Journal of Wildlife Diseases 15: 49-50.

--- 1980. Survival of Trichinella spiralis larvae in deep-frozen wolf tissue. Canadian Veterinary Journal 21: 38.

DIES, K.H., AND J.R. GUNSON. 1984. Prevalence and distribution of larvae of Trichinella sp. in cougars, Felis concolor L., and grizzly bears, Ursus arctos L., in Alberta. Journal of Wildlife Diseases 20: 242-244.

DIKMANS, G. 1932. Helminth parasites of deer. Journal of Parasitology 19:83.

- 1936. A note on Dictyocaulus from domestic and wild ruminants. Journal of the Washington Academy of Sciences 26: 298-303.

DODDS, D.G., AND J.S. MACKIEWICZ. 1961. Some parasites and diseases of snowshoe hares in Newfoundland. Journal of Wildlife Management 25: 409-414.

DOSTER, G.L., AND M. FRIEND. 1971. Spiculopteragia (Nematoda) from deer in North America. Journal of Parasitology 57: 468 . 
DUFF, D. 1914. The beaver fluke, Amphistomum subtriquetrum, Rudolphi. Transactions of the Royal Society of Canada 8 (series 3, section 4): 87-98.

DURRELL, W.B., AND W.D. BOLTON. 1957. Parasitosis in a musk ox. Journal of the American Veterinary Medical Association 131: 195-196.

DUSZYNSKI, D.W., W.M. SAMUEL, AND D.R. GRAY. 1977. Three new Eimeria spp. (Protozoa, Eimeriidae) from muskoxen, Ovibos moschatus, with redescriptions of $E$. faurei, $E$. granulosa, and $E$. ovina from muskoxen and from a Rocky Mountain bighorn sheep, Ovis canadensis. Canadian Journal of Zoology 55: 990-999.

EATON, R.D.P., AND D.C. SECORD. 1979. Some intestinal parasites of Arctic fox, Banks Island, N.W.T. Canadian Journal of Comparative Medicine 43: 229-230.

ERICKSON, A.B., AND P.R. HIGHBY. 1942. Parasites of the woodland caribou. Journal of Parasitology 28: 423.

FARLEY, J. 1962. Schistosomatium douthitti (Cort, 1914) Price, 1931 in Manitoba. Canadian Journal of Zoology 40: 131-133.

FIRLOTTE, W.R. 1948. A survey of the parasites of the brown Norway rat. Canadian Journal of Comparative Medicine 12: 187-191.

FLOOK, D.R., AND J.E. STENTON. 1969. Incidence and abundance of certain parasites in wapiti in the national parks of the Canadian Rockies. Canadian Journal of Zoology 47: 795-803.

FRANK, J.F. 1951. A study on the incidence of Trichinosis in wild rats in the Maritime provinces. Canadian Journal of Comparative Medicine 15: 279-283.

FRECHETTE, J.L., AND M.E. RAU. 1977. Helminths of the black bear in Quebec. Journal of Wildlife Diseases 13: 432-434. 1978. Seasonal changes in the prevalence of ova of Diphyllobothrium ursi and Baylisascaris transfuga in the feces of the black bear ( Ursus americanus). Journal of Wildlife Diseases 14: 342-344.

FREEMAN, R.S. 1956. Life history studies on Taenia mustelae Gmelin, 1790 and the taxonomy of certain taenioid cestodes from Mustelidae. Canadian Journal of Zoology 34: 219-242.

1958. On the epizootiology of Capillaria hepatica (Bancroft, 1893) in Algonquin Park, Ontario. Journal of Parasitology 44 (section 2): 33.

1960. Another hymenolepid with great morphological variation, Hymenolepis benneti n. sp. (Cestoda) from Napaeozapus insignis algonquinensis Prince. Canadian Journal of Zoology 38: 737-743.

1962. Studies on the biology of Taenia crassiceps (Zeder, 1800) Rudolphi, 1810 (Cestoda). Canadian Journal of Zoology 40: 969-990.

FREEMAN, R.S., A. ADORJAN, AND D.H. PIMLOTT. 1961. Cestodes of wolves, coyotes, and coyote-dog hybrids in Ontario. Canadian Journal of Zoology 39: 527-532.

FREEMAN, R.S., AND K.A. WRIGHT. 1960. Factors concerned with the epizootiology of Capillaria hepatica (Bancroft, 1893) (Nematoda) in a population of Peromyscus maniculatus in Algonquin Park, Canada. Journal of Parasitology 46: 373-382.

FYVIE, A. 1966. A review and current survey of the common diseases and parasites of wildlife in Ontario. Ontario Department of Lands and Forests Section Report (Wildlife) 63: 1-21.

GAMBLE, R.L., AND R.R. RIEWE. 1982. Infestations of the nematode Skrjabingylus nasicola (Leukart, 1842) in Mustela frenata (Lichtenstein) and M. erminea (L.) and some evidence of a paratenic host in the life cycle of this nematode. Canadian Journal of Zoology 60: 45-52.

GATES, C.C., AND W.M. SAMUEL. 1977. Prenatal infection of the Rocky Mountain bighorn sheep ( Ovis c. canadensis) of Alberta with the lungworm Protostrongylus spp. Journal of Wildlife Diseases 13: 248250.

GIBBS, H.C. 1957. On the role of rodents in the epidemiology of hydatid disease in the Mackenzie River basin. Canadian Journal of Comparative Medicine 21: 287-289. 1959. A redescription of Avitellina arctica Kolmakov, 1938 (Anoplocephalidae: Thysanosominae), from Rangifer arcticus arcticus in Northern Canada. Journal of Parasitology 45: 624-628.

- 1960. Disease investigation of barrenground caribou. Wildlife Management Bulletin, Canadian Wildlife Service, Ottawa, Series 1, No. 15: 119-135.

GIBBS, H.C., AND W.A. FULLER. 1959. Record of Wyominia tetoni Scott, 1941 from Ovis dalli in the Yukon Territory. Canadian Journal of Zoology 37: 815.

GIBBS, H.C., AND J.S. TENER. 1958. On some helminth parasites collected from the musk ox (Ovibos moschatus) in the Thelon Game 
Sanctuary, Northwest Territories.

Canadian Journal of Zoology 36: 531-532.

GIBSON, G.G., AND D.A. McKIEL. 1972.

Dracunculus insignis (Leidy, 1858) and

larval Eustrongylides sp. in a muskrat from

Ontario, Canada. Canadian Journal of

Zoology 50: 897-901.

GOBLE, F.C., AND E.C. DOUGHERTY. 1943.

Notes on the lungworms (Genus

Protostrongylus) of varying hares ( Lepus

americanus) in eastern North America.

Journal of Parasitology 29: 397-404.

GRANT, D.R., AND P.T.K. WOO. 1978a.

Comparative studies of Giardia spp. in

small mammals in southern Ontario. I.

Prevalence and identity of the parasites

with a taxonomic discussion of the genus.

Canadian Journal of Zoology 56: 1348-

1359.

1978b. Comparative studies of Giardia

spp. in small mammals in southern

Ontario. II. Host specificity and infectivity of stored cysts. Canadian Journal of Zoology 56: 1360-1366.

GRAY, J.B., W.M. SAMUEL, A.W. SHOSTAK, AND M.J. PYBUS. 1985. Varestrongylus alpenae (Nematoda: Metastrongyloidea) in white-tailed deer (Odocoileus virginianus) of Saskatchewan. Canadian Journal of Zoology 63: 1449-1454.

GREEN, H.U. 1949. Occurrence of Echinococcus granulosus in elk (Cervus canadensis nelsoni), Banff National Park. Canadian Field-Naturalist 63: 204-205.

GREGSON, J.D. 1937. Cysticercosis in deer. Parasitology 29: 409.

GUNSON, J.R., AND K.H. DIES. 1980. Sylvatic trichinosis in Alberta. Journal of Wildlife Diseases 16: 525-528.

GUPTA, R.P., AND H.C. GIBBS. 1971.

Infectivity of $D$. viviparus (moose strain) to calves. Canadian Veterinary Journal 12: 56.

GUPTA, S.P. 1962a. On a new trematode, Quinqueserialis zibethicai sp. nov., of the sub-family Notocotylinae from the duodenum of muskrat ( Ondatra zibethica) from Canada. Indian Journal of Helminthology 14: 66-70.

1962b. A redescription of Euparyphium melis (Schrank, 1788) Dietz, 1909 and Echinostoma revolutum (Froelich, 1802) Looss, 1899 parasitic in the intestine of mink ( Mustela vison) and muskrat ( Ondatra zibethica) from Canada. Indian Journal of Helminthology 14: 77-85.

HADWEN, S. 1916. A new host for Fasciola magna, Bassi, together with observations on the distribution of Fasciola hepatica, L., in Canada. Journal of the American

Veterinary Medical Association 2: 511-515. 1932. Helminth parasites of deer. Journal of Parasitology 19: 83.

HAIR, J.D., AND J.L. MAHRT. 1970. Eimeria albertensis n. sp. and $E$. borealis n. sp. (Sporozoa: Eimeriidae) in black bears Ursus americanus from Alberta. Journal of Protozoology 17: 663-664.

HARPER, T.A., R.A. RUTTAN, AND W.A. BENSON. 1955. Hydatid disease (Echinococcus granulosus) in Saskatchewan big game. Transactions of the 20th North American Wildlife Conference 198-208.

HILTON, D.F.J., AND J.L. MAHRT. 1971. Eimeria spermophilin. sp. and other Eimeria spp. (Sporozoa, Eimeriidae) from three species of Alberta Spermophilus (Rodentia, Sciuridae). Canadian Journal of Zoology 49: 699-701. - 1972a. Taxonomy of trypanosomes (Protozoa: Trypanosomatidae) of Spermophilus spp. (Rodentia: Sciuridae). Parasitology 65: 403-425. -1972b. Prevalence of Trypanosoma otospermophili (Protozoa: Trypanosomatidae) in five species of Spermophilus (Rodentia: Sciuridae). Parasitology 65: 427-432.

HNATIUK, J.M. 1966. First occurrence of Echinococcus multilocularis Leuckart, 1863 in Microtus pennsylvanicus in Saskatchewan. Canadian Journal of Zoology 44: 493.

- 1969. Occurrence of Echinococcus multilocularis Leuckart, 1863 in Vulpes fulva in Saskatchewan. Canadian Journal of Zoology 47: 264.

HOBBS, R.P. 1980. Interspecific interactions among gastrointestinal helminths in pikas of North America. American Midland Naturalist 103: 15-25.

HOBBS, R.P., AND W.M. SAMUEL. 1974. Coccidia (Protozoa, Eimeriidae) of the pikas Ochotona collaris, $O$. princeps, and $O$. hyperborea yesoensis. Canadian Journal of Zoology 52: 1079-1085.

HOBERG, E.P., AND S.G. MCGEE. 1982. Helminth parasitism in raccoons, Procyon lotor hirtus Nelson and Goldman, in Saskatchewan. Canadian Journal of Zoology 60: 53-57.

HOL.MES, J.C. 1961. The importance of coyotes 
(Canis latrans) in the maintenance of sylvatic echinococcosis: preliminary observations. Journal of Parasitology 47 (4, section 2): 55 .

1963. Helminth parasites of pine marten, Martes americana, from the District of MacKenzie. Canadian Journal of Zoology 41: 333 .

HOLMES, J.C., J.L. MAHRT, AND W.M. SAMUEL. 1971. The occurrence of Echinococcus multilocularis Leuckart, 1863 in Alberta. Canadian Journal of Zoology 49: 575-576.

HOLMES, J.C., AND R. PODESTA. 1968. The helminths of wolves and coyotes from the forested regions of Alberta. Canadian Journal of Zoology 46: 1193-1204.

HRUDKA, F., R.J. CAWTHORN, AND J.C. HAIGH. 1983. The occurrence of coccidia (Eimeriidae) in epididymal semen of a wapiti (Cervus canadensis nelsoni). Canadian Journal of Zoology 61: 1693 1699.

HUDSON, R., W.D. KITTS, AND P.J. BANDY. 1970. Monitoring parasite activity and disease in the Rocky Mountain bighorn by electrophoresis of seromucoids. Journal of Wildlife Diseases 6: 104-106.

JENNINGS, D.H., W. THRELFALL, AND D.G DODDS. 1982. Metazoan parasites and food of short-tailed weasels and mink in Newfoundland, Canada. Canadian Journal of Zoology 60: 180-183.

JENSEN, D.N. 1972. The life history of Scaphiostomum pancreaticum McIntosh, 1934 (Trematoda: Brachylaemidae). Canadian Journal of Zoology 50: 201-204.

JUNIPER, I. 1978. Morphology, diet, and parasitism in Quebec black bears. Canadian Field-Naturalist 92: 186-189

KARSTAD, L. 1963. Toxoplasma microti (The Morganism) in the muskrat ( Ondatra zibethica). Canadian Veterinary Journal 4: 249-251.

KARSTAD, L., AND D.O. TRAINER. 1969

Sarcocystis in white-tailed deer. Bulletin of the Wildlife Disease Association 5: 25-26.

KEITH, L.B., J.R. CARY, T.M. YUILL, AND I.M. KEITH. 1985. Prevalence of helminths in a cyclic snowshoe hare population. Journal of Wildlife Diseases 21: 233-253.

KELLY, A.I., L.R. PENNER, AND R.J. PICKARD. 1950. Sarcocystis in the moose. Journal of Mammalogy 31: 462-463.

KENNEDY, M.J. 1983. A revision of North American species of the genus

Parametorchis Skrjabin, 1913 (Trematoda:
Opisthorchiidae). Proceedings of the Helminthological Society of Washington 50 : 312-317.

KENNEDY, M.J., M.W. LANKESTER, AND J.B SNIDER. 1985. Paramphistomum cervi and Paramphistomum liorchis (Digenea: Paramphistomatidae) in moose, Alces alces, from Ontario, Canada. Canadian Journal of Zoology 63: 1207-1210.

KERR, G.R., AND J.C. HOLMES. 1966.

Parasites of mountain goats in west central Alberta. Journal of Wildlife Management 30: 786-790.

KINGSCOTE, A.A. 1934. Eimeria mustelae, n. sp. from Mustela vison. Journal of Parasitology 20: 252-253.

1947. Liver fluke control in the Burwash area. Report of the Ontario Veterinary College (1946): 34-35.

--1950. Liver rot (fascioloidiasis) in ruminants. Canadian Journal of Comparative Medicine 14: 203-208. 1957. Fish, wildlife, and zoo animals. Report of the Ontario Veterinary College for 1956: 26-27.

KNIGHT, I.M. 1951. Diseases and parasites of the muskrat (Ondatra zibethica) in British Columbia. Canadian Journal of Zoology 29: 188-214.

KNIGHT, R.A. 1974. Trichuris oreamnos sp. n. from the mountain goat, Oreamnos americanus (Blainville), in British Columbia, Canada, and a key to trichurids in North American ruminants. Journal of Parasitology 60: 275-279.

KNIGHT, R.A., AND L.S. UHAZY. 1973.

Redescription of Trichuris

(= Trichocephalus) schumakovitschi (Savinkova, 1967) from Canadian Rocky Mountain bighorn sheep ( Ovis canadensis canadensis). Journal of Parasitology 59: 136-140.

KO, R.C. 1972. The transmission of Ackertia marmotae Webster, 1967 (Nematoda: Onchocercidae) of groundhogs ( Marmota monax) by Ixodes cookei. Canadian Journal of Zoology 50: 437-450.

KO, R.C., AND R.C. ANDERSON. 1972. Tissue migration, growth, and morphogenesis of Filaroides martis (Nematoda:

Metastrongyloidea) in mink (Mustela vison). Canadian Journal of Zoology 50 : 1637-1649.

KRALKA, R.A. 1985. Range extension to Quebec for Protostrongylus boughtoni (Nematoda: Metastrongyloidea). The Canadian FieldNaturalist 99: 270. 
KRALKA, R.A., AND W.M. SAMUEL. 1984.

Experimental life cycle of Protostrongylus boughtoni (Nematoda: Metastrongyloidea), a lungworm of snowshoe hares, Lepus americanus. Canadian Journal of Zoology 62: 473-479.

LANKESTER, M.W. 1972. Diseases of moose in southeastern Manitoba. 8th North American Moose Conference Workshop, Thunder Bay, Ontario. Ontario Ministry for Natural Resources, Toronto. p. 42-59.

1974. Parelaphostrongylus tenuis

(Nematoda) and Fascioloides magna

(Trematoda) in moose of southeastern

Manitoba. Canadian Journal of Zoology 52: 235-239.

1977. Neurologic disease in moose caused by Elaphostrongylus cervi Cameron 1931 from caribou. Proceedings of the 13th North American Moose Conference Workshop, Jasper, Alberta. 177-190.

LANKESTER, M.W., AND R.C. ANDERSON. 1966. Small mammals as paratenic hosts of lungworms. Canadian Journal of Zoology 44: 341-342

1971. The route of migration and pathogenesis of Skrjabingylus spp. (Nematoda: Metastrongyloidea) in mustelids. Canadian Journal of Zoology 49 1283-1293.

LANKESTER, M.W., AND V.J. CRICHTON. 1972. Skrjabingylus lutrae n. sp. (Nematoda: Metastrongyloidea) from otter ( Lutra canadensis). Canadian Journal of Zoology 50: 337-340.

LANKESTER, M.W., V.J. CRICHTON, AND H.R. TIMMERMANN. 1976. A protostrongylid nematode (Strongylida: Protostrongylidae) in woodland caribou ( Rangifer tarandus caribou). Canadian Journal of Zoology 54: 680-684.

LANKESTER, M.W., AND T.H. NORTHCOTT. 1979. Elaphostrongylus cervi Cameron 1931 (Nematoda: Metastrongyloidea) in caribou ( Rangifer tarandus caribou) of Newfoundland. Canadian Journal of Zoology 57: 1384-1392.

LANKESTER, M.W., J.B. SNIDER, AND R.E. JERRARD. 1979. Annual maturation of Paramphistomum cervi (Trematoda: Paramphistomatidae) in moose, Alces alces L. Canadian Journal of Zoology 57: 23552357.

LAW, R.G. 1933a. Hookworm infection in foxes. Bulletin of the Ontario Department for Game Fish 5: 1-39.

1933b. Tapeworm in rabbits. Canadian
Field-Naturalist 47: 142.

LAW, R.G., AND A.H. KENNEDY. 1932.

Parasites of fur-bearing animals. Bulletin of the Ontario Department for Game Fish 4: 1-30.

LAW, R.G., AND A.C. SECORD. 1931. Control of lungworm in silver foxes. North American Veterinarian 12: 42-44.

LEE, B.L., AND R.S. DORNEY. 1971. Eimeria ontarioensis n. sp., E. confusa Joseph, 1969 and Eimeria sp. (Protozoa: Eimeriidae) from the Ontario gray squirrel Sciurus carolinensis. Journal of Protozoology 18: 587-592.

LEE, C.-F. 1969. Larval Echinococcus multilocularis Leuckart, 1863 in the southern Interlake area in Manitoba, Canada. Canadian Journal of Zoology 47: 733-734.

LEE, T.D.G., AND K.A. WRIGHT. 1978. The morphology of the attachment and probable feeding site of the nematode Trichuris muris (Schrank, 1788) Hall, 1916. Canadian Journal of Zoology 56: 1889 1905.

LEIBY, P.D., G. LUBINSKY, AND W. GALAUGHER. 1969. Studies on sylvatic echinococcosis. II. The occurrence of Echinococcus multilocularis Leuck. 1863 in Manitoba. Canadian Journal of Zoology 47: 135-138.

LEPP, D.L., K.S. TODD, JR., AND W.M. SAMUEL. 1972. Four new species of Eimeria (Protozoa: Eimeriidae) from the pika Ochotona princeps from Alberta and $O$. pallasi from Kazakhstan. Journal of Protozoology 19: 192-195.

- 1973. Eimeria banffensis n. sp. (Protozoa: Eimeriidae) from the pika Ochotona princeps from Alberta. Transactions of the American Microscopical Society 92: 305307.

LESTER, R.J.G., AND R.S. FREEMAN. 1976. Survival of two trematode parasites (Diplostomum spp.) in mammalian eyes and associated pathology. Canadian Journal of Ophthalmology 11: 229-234.

LEVINE, N.D., J.O. CORLISS, F.E.G. COX, et al 1980. A newly revised classification of the protozoa. Journal of Protozoology 27: 37 58.

LEVINE, N.D., AND V. IVENS. 1963. Eimeria siniffi sp. n. and $E$. arizonensis (Protozoa: Eimeriidae) from deermice in British Columbia. Journal of Parasitology 49: 660661.

LINCOLN, R.C., AND R.C. ANDERSON. 1973. 
The relationship of Physaloptera maxillaris (Nematoda: Physalopteroidea) to skunk (Mephitis mephitis). Canadian Journal of Zoology 51: 437-441.

LOW, W.A. 1976. Parasites of woodland caribou in Tweedsmuir Provincial Park, British Columbia. Canadian Field-Naturalist 90: 189-191.

LUBINSKY, G. 1956. On the probable presence of parasitic liver cirrhosis in Canada. Canadian Journal of Comparative Medicine 20: $457-465$.

1957. List of helminths from Alberta rodents. Canadian Journal of Zoology 35: 623-627.

1958. On the 12-hooked oncospheres of Canadian strains of Echinococcus. Canadian Journal of Zoology 36: 883-887.

LUBINSKY, G., AND R. BARON. 1970. Taenia crassiceps (Zeder, 1800) in metropolitan Winnipeg, Manitoba. Canadian Journal of Zoology 48: 1144-1145.

LUBINSKY, G., B.R. JACOBSEN, AND R.W. BARON. 1971. Wildlife foci of Capillaria hepatica infections in Manitoba. Canadian Journal of Zoology 49: 1201-1202.

MACDONALD, D.W., W.M. SAMUEL, AND J.O.C. HUNTER. 1976. Haemonchosis in a captive muskox calf. Canadian Veterinary Journal 17: 138-139.

MACE, T.F. 1976. Lesions in mink (Mustela vison) infected with giant kidney worm (Dioctophyma renale). Journal of Wildlife Diseases 12: 88-92.

MACE, T.F., AND R.C. ANDERSON. 1975. Development of the giant kidney worm, Dioctophyma renale (Goeze, 1782)

(Nematoda: Dioctophymatoidea). Canadian Journal of Zoology 53: 1552-1568.

MACE, T.F., AND C.D. SHEPARD. 1981.

Helminths of a Vancouver Island marmot, Marmota vancouverensis Swarth, 1911, with a description of Diandrya vancouverensis $\mathrm{sp}$. nov. (Cestoda: Anoplocephalidae). Canadian Journal of Zoology 59: 790-792.

MacKINNON, B.M., AND M.D.B. BURT. 1978. Platyhelminth parasites of muskrats (Ondatra zibethica) in New Brunswick. Canadian Journal of Zoology 56: 350-354.

MAHRT, J.L., AND S.J. CHAI. 1970. Prevalence of coccidia in red squirrels ( Tamiasciurus hudsonicus) in Alberta. Canadian Journal of Zoology 48: 606.

1972. Parasites of red squirrels in Alberta, Canada. Journal of Parasitology 58: $639-640$.
MAHRT, J.L., AND D.D. COLWELL. 1980. Sarcocystis in wild ungulates in Alberta. Journal of Wildlife Diseases 16: 571-576.

MALTAIS, P.M., AND E.A. OUELLETTE. 1979. Dirofilaria scapiceps (Leidy, 1886) from the snowshoe hare, Lepus americanus struthopus Bangs, in New Brunswick, Canada. Le Naturaliste Canadien 106: 561-562.

McGEE, S.G. 1980. Helminth parasites of squirrels (Sciuridae) in Saskatchewan. Canadian Journal of Zoology 58: 2040 2050.

McKENZIE, C.E., AND H.E. WELCH. 1979. Parasite fauna of the muskrat, Ondatra zibethica (Linnaeus, 1766), in Manitoba, Canada. Canadian Journal of Zoology 57: 640-646.

McLEOD, J.A. 1933. A parasitological survey of the genus Citellus in Manitoba. Canadian Journal of Research, Section D, Zoological Sciences 9: 108-127.

MeNEILL, M.A., M.E. RAU, AND F. MESSIER. 1984. Helminths of wolves (Canis lupus L.) from southwestern Quebec. Canadian Journal of Zoology 62: 1659-1660.

MEASURES, L.N., AND R.C. ANDERSON. 1983a. Characteristics of natural infections of the stomach worm, Obeliscoides cuniculi (Graybill), in lagomorphs and woodchucks in Canada. Journal of Wildlife Diseases 19: 219-224.

1983b. New subspecies of the stomach worm, Obeliscoides cuniculi (Graybill), of lagomorphs. Proceedings of the Helminthological Society of Washington 50 : 1-14.

1984. Hybridization of Obeliscoides cuniculi cuniculi (Graybill, 1923) Graybill, 1924 and Obeliscoides cuniculi multistriatus Measures and Anderson, 1983. Proceedings of the Helminthological Society of Washington 51: 179-186.

MILLER, M.J. 1940. A new trematode, Fibricola laruei, from the raccoon in Canada. Canadian Journal of Research, Section D, Zoological Sciences 18: 333-335.

MOYNIHAN, I.W., AND I.W. MUSFELDT. 1949. A study of the incidence of trichinosis in rats in British Columbia. Canadian Journal of Comparative Medicine 13: 152155.

PATERSON, W.B., AND P.T.K. WOO. 1984. The development of the culture and bloodstream forms of three Trypanosoma ( Schizotrypanum) spp. (Protista: Zoomastigophorea) from bats in Cimex 
lectularius (Hemiptera: Cimicidae).

Canadian Journal of Zoology 62: 1581-1587.

1954. The life cycles of Alaria

arisaemoides Augustine and Uribe, 1927,

Alaria canis LaRue and Fallis, 1936

(Trematoda: Diplostomidae), parasites of

the red fox, Vulpes fulva (Desmarest).

Journal of Parasitology 40 (section 2): $37-$

38.

1956. Studies on the life cycles and

morphology of the larval stages of Alaria

arisaemoides Augustine and Uribe, 1927

and Alaria canis LaRue and Fallis, 1936

(Trematoda: Diplostomidae). Canadian

Journal of Zoology 34: 295-387.

PENCE, D.B., H.P. SAMOIL, AND J.E. STONE.

1978. Spirocercid stomach worms

(Nematoda: Spirocercidae) from wild felids

in North America. Canadian Journal of

Zoology 56: 1032-1042.

PETERSON, R.L. 1955. North American Moose.

University of Toronto Press, Toronto (See pp. 181-192).

PLATT, T.R. 1978. A report of Polymorphus paradoxus (Acanthocephala) in Microtus pennsylvanicus from Hastings Lake, Alberta (Canada). Proceedings of the Helminthological Society of Washington 45: 255.

PLATT, T.R., AND W.M. SAMUEL. 1978a. Parelaphostrongylus odocoilei: Life cycle in experimentally infected cervids including the mule deer, Odocoileus h. hemionus. Experimental Parasitology 46: 330-338. 1978b. A redescription and neotype designation for Parelaphostrongylus odocoilei (Nematoda: Metastrongloidea). Journal of Parasitology 64: 226-232.

PLEDGER, D.J., W.M. SAMUEL, AND D.A. CRAIG. 1980. Black flies (Diptera, Simuliidae) as possible vectors of legworm (Onchocerca cervipedis) in moose of central Alberta. Proceedings of the North

American Moose Conference Workshop 16: 171-202.

POOLE, B.C., K. CHADEE, AND T.A. DICK. 1983. Helminth parasites of pine marten, Martes americana (Turton), from Manitoba, Canada. Journal of Wildlife Diseases 19: 10-13.

POOLE, J.B. 1952. A survey of the incidence of trichinosis in rats in BC. Canadian Journal of Comparative Medicine 16: 291-293.

PRESIDENTE, P.J.A., AND L.H. KARSTAD. 1975a. Hepatozoon sp. infection in mink from southwestern Ontario. Journal of Wildlife Diseases 11: 479-481.
PRESIDENTE, P.J.A., AND R.O. RAMSDEN.

1975b. Paragonimus kellicotti infection in wild carnivores in southwestern Ontario: II. Histopathologic features. Journal of Wildlife Diseases 11: 364-375.

PRICE, E.W. 1929. Two new species of trematodes of the genus Parametorchis from fur-bearing animals. Proceedings of the United States National Museum 76: 1-5. - 1931. Hepaticola hepatica in liver of Ondatra zibethica. Journal of Parasitology 18: 51. 1932. The occurrence of Euparyphium inerme (Fuhrmann) in North America. Journal of Parasitology 19: 311. 1934. A new trematode from a beaver. Proceedings of the Helminthological Society of Washington 1: 1-2.

PYBUS, M.J., W.J. FOREYT, AND W.M. SAMUEL. 1984. Natural infections of Parelaphostrongylus odocoilei (Nematoda: Protostrongylidae) in several hosts and locations. Proceedings of the Helminthological Society of Washington 51: 338-340.

PYBUS, M.J., AND W.M. SAMUEL. 1980. Pathology of the muscleworm

Parelaphostrongylus odocoilei (Nematoda: Metastrongyloidea), in moose. Proceedings of the North American Moose Conference Workshop 16: 152-170.

1981. Nematode muscleworm from whitetailed deer of southeastern British Columbia. Journal of Wildlife Management 45: 537-542.

QUINN, P.J., R.O. RAMSDEN, AND D.H. JOHNSTON. 1976. Toxoplasmosis: A serological survey in Ontario wildlife. Journal of Wildlife Diseases 12: 504-510.

RAMSDEN, R.O., AND P.J.A. PRESIDENTE. 1975. Paragonimus kellicotti infection in wild carnivores in southwestern Ontario: I. Prevalence and gross pathologic features. Journal of Wildlife Diseases 11: 136-141.

RAU, M.E., AND F.R. CARON. 1979. Parasiteinduced susceptibility of moose to hunting. Canadian Journal of Zoology 57: 24662468.

RAU, M.E., AND C.E. TANNER. 1972. Echinococcus multilocularis in the cotton rat. Asexual proliferation following the intraperitoneal inoculation of graded doses of protoscolices. Canadian Journal of Zoology 50: 941-946.

1973. Echinococcus multilocularis in the cotton rat. The effect of preexisting subcutaneous cysts on the development of a 
subsequent intraperitoneal inoculum of protoscolices. Canadian Journal of Zoology 51: $55-59$.

RAUSCH, R.L. 1952b. Studies on the helminth fauna of Alaska. XI. Helminth parasites of microtine rodents-taxonomic considerations. Journal of Parasitology 38 : 415-444.

RAUSCH, R.L., AND J.D. TINER. 1948. Studies on the parasitic helminths of the North Central States. I. Helminths of sciuridae. American Midland Naturalist 39: 728-747.

RAUSCH, R.L., AND J.P. TINER. 1949. Studies on the parasitic helminths of the North Central States. II. Helminths of voles ( Microtus ssp.) Preliminary report. American Midland Naturalist 41: 665-694. RIEDEL, D. 1975. Reproduction, structure and host specificity of Trypanosoma ( Herpetosoma) tamiasi sp. n. from the eastern chipmunk, Tamias striatus. Journal of Protozoology 22: 8-17.

RIGBY, M.D., AND W. THRELFALL. 1981. Helminth parasites of the muskrat (Ondatra zibethicus (L.)) in Newfoundland. Canadian Journal of Zoology 59: 2172 2176.

RITCEY, R.W., AND R.Y. EDWARDS. 1958. Parasites and diseases of the Wells Gray moose herd. Journal of Mammalogy 39: 139-145.

SAMOIL, H.P., AND W.M. SAMUEL. 1977a. Experimental study of Eimeria robertsoni (Protozoa: Eimeriidae) in the snowshoe hare, Lepus americanus. Journal of Parasitology 63: 203-205. - 1977b. Description of nine species of Eimeria (Protozoa, Eimeriidae) in the snowshoe hare, Lepus americanus, of central Alberta. Canadian Journal of Zoology 55: 1671-1683.

SAMUEL, W.M. 1972a. Taenia krabbei in the musculature of moose: a review. Paper presented at 8th North American Moose Conference Workshop, Thunder Bay, Ontario, February, 1972. pp. 18-41.

- 1972b. Encounters between hunters and parasites of moose in Alberta. Occasional paper No. 2. Alberta Fish and Wildlife Division $21 \mathrm{pp}$.

SAMUEL, W.M., M.W. BARRETT, AND G.M. LYNCH. 1976. Helminths in moose of Alberta. Canadian Journal of Zoology 54: 307-312.

SAMUEL, W.M., AND D.R. GRAY. 1974.

Parasitic infection in muskoxen. Journal of Wildlife Management 38: 775-782.
SAMUEL, W.M., AND J.C. HOLMES. 1974.

Search for elaphostrongyline parasites in cervids from Alberta. Canadian Journal of Zoology 52: 401-403.

SAMUEL, W.M., W.K. HALL, J.G. STELFOX, AND W.D. WISHART. 1978. Parasites of moutain goat, Oreamnos americanus (Blainville), of west central Alberta with a comparison of the helminths of mountain goat and Rocky Mountain bighorn sheep, Ovis c. canadensis Shaw. Proceedings of the 1st International Mountain Goat

Symposium, Kalispell, Montana 1: 212-215.

SAMUEL, W.M., S. RAMALINGAM, AND L.N.

CARBYN. 1978. Helminths in coyotes (Canis latrans Say), wolves (Canis lupus L.), and red foxes (Vulpes vulpes L.) of southwestern Manitoba. Canadian Journal of Zoology 56: 2614-2617.

SAUNDERS, B.P. 1973. Meningeal worm in white-tailed deer in northwestern Ontario and moose population densities. Journal of Wildlife Management 37: 327-330.

SCHAD, G.A. 1954. Helminth parasites of mice in northeastern Quebec and the coast of Labrador. Canadian Journal of Zoology 32 : 215-224.

1956. Helminths recovered from deer mice, Peromyscus maniculatus, trapped in the Morgan Arboretum, Macdonald College, province of Quebec. Canadian Journal of Zoology 34: 208.

SCHILLER, E.L. 1952. Studies on the helminth fauna of Alaska. X. Morphological variation in Hymenolepis horrida (von Linstow, 1901) (Cestoda: Hymenolepididae). Journal of Parasitology 38: 554-568.

SCHMIDT, G.D. 1986. CRC Handbook of Tapeworm Identification. $688 \mathrm{pp}$.

SCHMITT, N., E.J. BOWMER, P.C. SIMON, A.S. ARNEIL, AND D.A. CLARK. 1972.

Trichinosis from bear meat and adulterated pork products: a major outbreak in British Columbia, 1972. Can. Med. Assoc. J. 107: 1087-1091.

SCHMITT, N., J.M. SAVILLE, J.A. GREENWAY, P.L. STOVELL, L. FRIIS, AND L. HOLE. 1978. Sylvatic trichinosis in British Columbia. Public Health Report 93: 189193.

SCHOWALTER, D.B., J.O. IVERSEN, L.C. CORNER, AND J.R. GUNSON. 1980. Prevalence of antibodies to Toxoplasma gondii in striped skunks from Saskatchewan and Alberta. Journal of Wildlife Diseases 16: 189-194.

SHANTA, C.S., AND E. MEEROVITCH. 1967a. 
The life cycle of Trichinella spiralis. I. The intestinal phase of development. Canadian Journal of Zoology 45: 1255-1260.

1967b. The life cycle of Trichinella spiralis. II. The muscle phase of development and its possible evolution. Canadian Journal of Zoology 45: 1261 1267.

SHOSTAK, A.W., AND W.M. SAMUEL. 1984.

Moisture and temperature effects on survival and infectivity of first-stage larvae of Parelaphostrongylus odocoilei and $P$. tenuis (Nematoda: Metastrongyloidea). Journal of Parasitology 70: 261-269.

SIMON, C.E. 1921. Giardia in field mice in Nova Scotia. Journal of Parasitology 7: 190.

SKINKER, M.S. 1935. A redescription of Taenia tenuicollis Rudolphi, 1819, and its larva, Cysticercus talpae Rudolphi, 1819. Parasitology 27: 175-185.

SLEGGS, G.F. 1925. A strongyloid nematode, Warrenius bifurcatus $\mathrm{n}$. sp., from the Richardson ground squirrel. Parasitology 17: 410-416.

SMITH, F.R., AND W. THRELFALL. 1973.

Helminths of some mammals from Newfoundland. American Midland Naturalist 90: 215-218.

SMITH, H.J. 1967. The sclerostome, Castorstrongylus castoris Chapin, 1925, in two New Brunswick beaver. Canadian Journal of Zoology 45: 893 .

1978b. Parasites of red foxes in New Brunswick and Nova Scotia. Journal of Wildlife Diseases 14: 366-370.

SMITH, H.J., AND R. McG. ARCHIBALD. 1967. On the incidence of gastrointestinal parasites in Nova Scotia beaver. Canadian Journal of Zoology 45: 659-661.

SMITH, H.J., R. McG. ARCHIBALD, AND A.H. CORNER. 1964. Elaphostrongylosis in maritime moose and deer. Canadian Veterinary Journal 5: 287-296.

SOON, B.-L., AND R.S. DORNEY. 1969a. Observations on a coccidium (Eimeria dorneyi) from the northern flying squirrel in Ontario. Bulletin of the Wildlife Disease Association 5: 37-38.

SPRENT, J.F.A. 1952. On an Ascaris parasite of the fisher and marten, Ascaris devosi sp. nov.. Proceedings of the Helminthological Society of Washington 19: 27-37.

STAFFORD, J. 1905. Trematodes from Canadian vertebrates. Zoologischer Anzeiger 28: 681694.

STELFOX, J.G. 1962. Liver, lungs and larvae.
Parasites and diseases in moose, deer and elk in Alberta. Alberta Lands, Forests, and Wildlife Annual Report 5: 5-12.

STOCK, T.M., AND M.W. BARRETT. 1983.

Helminth parasites of the gastrointestinal tracts and lungs of moose (Alces alces) and wapiti (Cervus elaphus) from Cypress Hills, Alberta, Canada. Proceedings of the Helminthological Society of Washington 50: 246-251.

STOCKDALE, P.H.G. 1970a. Pulmonary lesions in mink with a mixed infection of Filaroides martis and Perostrongylus pridhami. Canadian Journal of Zoology 48: 757-759. 1970b. The development, route of migration, and pathogenesis of

Perostrongylus pridhami in mink. Journal of Parasitology 56: 559-566.

STOCKDALE, P.H.G., AND R.C. ANDERSON. 1970. The development, route of migration, and pathogenesis of Filaroides martis in mink. Journal of Parasitology 56: 550-558.

STOCKDALE, P.H.G., M.A. FERNANDO, AND R. CRAIG. 1974. The development, route of migration, and pathogenesis of Crenosoma mephitidis in the skunk (Mephitis mephitis). Canadian Journal of Zoology 52: 681-685.

SWALES, W.E. 1933b. A review of Canadian helminthology: II. Additions to Part I, as determined from a study of parasitic helminths collected in Canada. Canadian Journal of Research, Section D, Zoological Sciences 8: 478-482.

- 1934. Skrjabinema oreamni sp. nov., a nematode parasite of Oreamnos americanus and Rangifer sp. in Canada. Canadian Journal of Research, Section D, Zoological Sciences 10: 527-531.

- 1935. The life cycle of Fascioloides magna (Bassi, 1875), the large liver fluke of ruminants, in Canada with observations on the bionomics of the larval stages and the intermediate hosts, pathology of Fascioloidiasis magna, and control measures. Canadian Journal of Research, Section D, Zoological Sciences 12: 177-215. 1936. Further studies on Fascioloides magna (Bassi, 1875) Ward, 1917, as a parasite of ruminants. Canadian Journal of Research Section D, Zoological Sciences 14: 83-95.

1938. Skrjabingylus nasicola (Leuckart, 1842) Petrow, 1927, a nematode parasitic in the frontal sinuses of American Mustelidae. Livre Jubilar do Professor Lauro Travassos, Rio de Janeiro, pp. 455-458. 
SWEATMAN, G.K. 1952a. Distribution and incidence of Echinococcus granulosus in man and other animals with special reference to Canada. Canadian Journal of Public Health 43: 480-486.

-1952b. Endoparasites of muskrats in the vicinity of Hamilton, Ontario. Journal of Mammalogy 33: 248-250.

SWEATMAN, G.K., AND T.C. HENSHALL. 1962. The comparative biology and morphology of Taenia ovis and T. krabbei, with observations on the development of $T$. ovis in domestic sheep. Canadian Journal of Zoology 40: 1287-1311.

SWEATMAN, G.K., AND P.J.G. PLUMMER. 1957. The biology and pathology of the tapeworm Taenia hydatigena in domestic and wild hosts. Canadian Journal of Zoology 35: 93-109.

SWEATMAN, G.K., AND R.J. WILLIAMS. 1963. Comparative studies on the biology and morphology of Echinococcus granulosus from domestic livestock, moose and reindeer. Parasitology 53: 339-390.

THRELFALL, W. 1967. Parasites of moose (Alces alces) in Newfoundland. Journal of Mammalogy 48: 668-669.

1969. Further records of helminths from Newfoundland mammals. Canadian Journal of Zoology 47: 197-201.

TINER, J.D. 1950. Two new species of Trichuris from North America, with redescriptions of Trichuris opaca and Trichuris leporis (Nematoda: Aphasmidea). Journal of Parasitology 36: 350-355.

TIZARD, I.R., J.B. BILLETT, AND R.O.

RAMSDEN. 1976. The prevalence of antibodies against Toxoplasma gondii in some Ontario mammals. Journal of Wildlife Diseases 12: 322-325.

TIZARD, I.R., J. HARMESON, AND C.H. LAI. 1978. The prevalence of serum antibodies to Toxoplasma gondii in Ontario mammals. Canadian Journal of Comparative Medicine 42: $177-183$.

UHAZY, L.S., J.C. HOLMES, AND J.G. STELFOX. 1973. Lungworms in the Rocky Mountain bighorn sheep of western Canada. Canadian Journal of Zoology 51: 817-824.

UHAZY, L.S., J.L. MAHRT, AND J.C. HOLMES. 1971. Coccidia of Rocky Mountain bighorn sheep in Western Canada. Canadian Journal of Zoology 49: 1461-1464.

WALKER, M.L., AND W.W. BECKLUND. 1970. Checklist of the internal and external parasites of deer, Odocoileus hemionus and $O$. virginianus, in the United States and
Canada. Special Publication 1. Index Catalogue of Medical and Veterinary Zoology, National Animal Parasite Laboratory, Veterinary Science Research Division Agricultural Research Services 145.

WATSON, E.A., AND S. HADWEN. 1912. Trypanosomes found in Canadian mammals. Parasitology 5: 21-26.

WATSON, T.G. 1981. Growth of Metorchis conjunctus (Cobbold, 1860) Looss, 1899 (Trematoda: Opisthorchiidae) in the bile ducts of various definitive hosts. Canadian Journal of Zoology 59: 2014-2019.

WEBSTER, G.A., AND T.W.M. CAMERON. 1961. Observations on experimental infections with Echinococcus in rodents. Canadian Journal of Zoology 39: 877-892.

WEBSTER, G.A., AND R.W. WOLFGANG. 1956. Alaria canadensis sp. nov. and Euryhelmis pyriformis sp. nov. from the skunk Mephitis mephitis in Quebec. Canadian Journal of Zoology 34: 595-601.

WEBSTER, W.A. 1964. Crenosoma canadensis n. sp. (Nematoda: Metastrongylidae) from the lungs of skunks. Canadian Journal of Zoology 42: 807-809.

1965. Skrjabingylus magnus n. sp. (Nematoda: Trichostrongylidae) from the skunk, Mephitis mephitis. Canadian Journal of Zoology 43: 229-231.

- 1966a. Microfilariae in Ontario skunks: Final report. Canadian Journal of Zoology 44: 495.

- 1966b. A morphological description of Capillaria michiganensis Read, 1949: Male and female. Canadian Journal of Zoology 44: 982-983.

- 1967a. Filaroides mephitis n. sp.

(Metastrongyloidea: Filaroididae) from the lungs of eastern Canadian skunks. Canadian Journal of Zoology 45: 145-147.

-1967b. Ackertia marmotaen. sp.

(Filarioidea: Onchocercinae) from the groundhog (Marmota monax). Canadian Journal of Zoology 45: 277-283.

- 1974. Records of cestodes in varying lemmings and an arctic fox from Bathurst Island, Northwest Territories. Canadian Journal of Zoology 52: 1425-1426.

1980. The direct transmission of Andersonstrongylus captivensis Webster 1978 (Metastrongyloidea:

Angiostrongylidae) in captive skunks Mephitis mephitis (Schreber). Canadian Journal of Zoology 58: 1200-1203.

WEBSTER, W.A., AND M. BEAUREGARD. 
1964. Microfilaria mephitis $\mathrm{n}$. sp.

(Filarioidea: Dipetalonematidae) from the brain of a skunk: with notes on its occurrence in Ontario. Canadian Journal of Zoology 42: 811-815.

1965. Dipetalonema mephitis n. comb.

(Microfilaria mephitis: Webster and

Beauregard, 1964) from the skunk, Mephitis mephitis. Canadian Journal of Zoology 43: 325-332.

WEBSTER, W.A., AND G.A. CASEY. 1970. The occurrence of Dracunculus insignis (Leidy, 1858) Chandler, 1942 in a skunk from Ontario, Canada. Journal of Wildlife Diseases 6: 71.

1973. Studies on the parasites of Chiroptera. III. Helminths from various bat species collected in British Columbia. Canadian Journal of Zoology 51: 633-636.

WEBSTER, W.A., AND R.R. MACKAY. 1969.

Two new host records for Nematodirus species. Canadian Journal of Zoology 47: 1411.

WEBSTER, W.A., AND J. ROWELL. 1980. Some helminth parasites from the small intestine of free-ranging muskoxen Ovibos moschatus (Zimmermann) of Devon and Ellesmere Islands, Northwest Territories. Canadian Journal of Zoology 58: 304-305.

WESTERMANN, J.E.M., AND V.E.

ENGELBERT. 1969. Numbers of granulocytes on thymic imprints of rabbits of different ages and degrees of coccidial parasitism. Canadian Journal of Zoology 47: 1355-1362.

WOBESER, G. 1976. Besnoitiosis in a woodland caribou. Journal of Wildlife Diseases 12: 566-571.

WOBESER, G., A.A. GAJADHAR, AND H.M.

HUNT. 1985. Fascioloides magna:

Occurrence in Saskatchewan and distribution in Canada. Canadian Veterinary Journal 26: 241-244.

WOBESER, G., AND T.W. ROCK. 1973.

Capillaria hepatica (Nematoda:

Trichuridae) in a coyote (Canis latrans). Journal of Wildlife Diseases 9: 225-226.

WOBESER, G., W. RUNGE, AND R.R.

STEWART. 1983. Metorchis conjunctus (Cobbold, 1860) infection in wolves ( Canis

lupus), with pancreatic involvement in two animals. Journal of Wildlife Diseases 19: 353-356.

WOBESER, G., AND J.C.L. SCHUH. 1979.

Microsporidal encephalitis in muskrats. Journal of Wildlife Diseases 15: 413-417.
WOLFGANG, R.W. 1956. Dochmoides yukonensis sp. nov. from the brown bear (Ursus americanus) in the Yukon. Canadian Journal of Zoology 34: 21-27.

WOO, P.T.K., D.R. GRANT, AND L. McLEAN. 1980. Trypanosomes of small mammals in southern Ontario. Canadian Journal of Zoology 58: 567-571.

WRIGHT, K.A. 1961. Observations on the life cycle of Capillaria hepatica (Bancroft, 1893) with a description of the adult. Canadian Journal of Zoology 38: 167-182.

1972. The fine structure of the esophagus of some trichuroid nematodes. I. The stichosome of Capillaria hepatica, Trichuris myocastoris, and Trichuris vulpis.

Canadian Journal of Zoology 50: 319-324.

YAMAGUTI, S. 1959. Systema Helminthum. Volume 2. The Cestoda of Vertebrates. 860 pp. Interscience Publishing Incorporated, New York. 1971. Synopsis of Digenetic Trematodes of Vertebrates. 1074 pp. Keigaku Publishing Company, Tokyo.

ZYLL DE JONG, C.G. VAN. 1966. Parasites of the Canada lynx, Felis ( Lynx) canadensis (Kerr). Canadian Journal of Zoology 44: 499-509. 


\section{SUPPLEMENTARY READING}

AMEEL, D.J. 1932. The muskrat, a new host for Paragonimus. Science 75: 382.

ANDERSON, R.C. 1951. A species of Dirofilaria from the black bear (Euarctos a. americana) Journal of Parasitology (suppl.). 37: 25. - 1965b. Cerebrospinal nematodiasis (Pneumostrongylus tenuis) in North American cervids. Transactions of the 13th North American Wildlife Conference 30: 156-167.

1968. The pathogenesis and transmission of neurotropic and accidental nematode parasites of the central nervous system of mammals and birds. Helminthological Abstracts 37: 191-210.

1971a. Lungworms, p. 81-126. In: Davis, J.W. and R. C. Anderson (eds.) Parasitic disease of wild animals. Iowa State University Press.

- 1972. The ecological relationships of meningeal worm and native cervids in North America. Journal of Wildlife Diseases 8: 304-310.

ANDERSON, R.C., AND M.W. LANKESTER. 1974. Infectious and parasitic diseases and arthropod pests of moose in North America. Nature Canada (Ottawa) 101: 23-50.

ANONYMOUS 1932. Report of the experimental fur farm. Ontario

Department of Game Fish. Annual Report 25 (1931): 8-43.

- 1967. Status of wildlife research projects for the year, 1966. Ontario Department of Lands and Forests Section Report (Wildlife) 65: 1-52. (See pp. 11-35).

BARTLETT, C.M. 1983b. Cercopithifilaria leporinus n. sp. (Nematoda: Filarioidea) from the snowshoe hare (Lepus americanus Erxleben) (Lagomorpha) in Canada. Annales de Parasitologie Humaine et Comparee 58: 275-283.

BAUGH, S.C. 1958. Contributions to our knowledge of digenetic trematodes. Proceedings of the National Academy of Sciences, India, Section B (Biological Sciences) 28: 205-226.

BECKLUND, W.W., AND C.M. SENGER. 1967. Parasites of Ovis canadensis canadensis in Montana with a checklist of the internal and external parasites of the Rocky Mountain bighorn sheep in North America. Journal of Parasitology 53: 157-165.

BECKLLND, W.W., AND M.L. WALKER. 1970.
Checklist of the internal and external parasites of deer, Odocoileus hemionus and $O$. virginianus, in the United States and Canada. Index-Catalogue of Medical and Veterinary Zoology. Special Publication No. 1.45 pp.

BERNARD, M.A. 1971. Dirofilariasis in Canada. Canadian Veterinary Journal 12: 139.

BROUGHTON, E., AND L.P.E. CHOQUETTE. 1969. Additional information and comments on disease conditions and parasites of barren-ground caribou. Transactions of the 33rd Federal-Provincial Wildlife Conference, Edmonton, Alta. pp. 30-39.

BROWN, J.H., AND G.D. ROY. 1943. The Richardson ground squirrel, Citellus richardsoni Sabine, in southern Alberta: its importance and control. Science in Agriculture Review of Agronomy Canada 24: 176-197.

BROWN, M., ET AL. 1949. A note on trichinosis in animals of the Canadian Northwest Territories. Canadian Journal of Public Health 40: 20-21.

CAMERON, T.W.M. 1938. On the morphology and parasitic development of Travassosius rufus Khalil, 1922, a trichostrongyle parasite of the Canadian beaver ( Castor canadensis canadensis). Libro Jubilar L. Travassos, Rio de Janeiro. pp. 103-106. - 1944. The morphology, taxonomy and life history of Metorchis conjunctus (Cobbold, 1860). Canadian Journal of Research Section D, Zoological Sciences 22: 6-16.

CAWTHORN, R.J., AND R.J. BROOKS. 1985. Histological observations on precystic merogony and metrocyte formation of Sarcocystis rauschorum (Apicomplexa: Sarcocystidae) in varying lemmings, Dicrostonyx richardsoni. Canadian Journal of Zoology 63: 2907-2912.

CHITWOOD, B.G. 1932. The occurrence of Uncinaria stenocephala from the fisher, Mustela sp. Journal of Parasitology 19: 307.

CRUM, J.M., R. FAYER, AND A.K. PRESTWOOD. 1981. Sarcocystis spp. in white-tailed deer. I. Definitive and intermediate host spectrum with a description of $S$. odocoileocanis $\mathrm{n}$. sp. Journal of Wildlife Diseases 17: 567-578

DEROTH, L., AND J.L. FRECHETTE. 1975. 
Dracunculus insignis (Leidy, 1858) chez un berger allemand. Canadian Veterinary Journal 16: 176-178.

DE VOS, A. 1957. Pregnancy and parasites of marten. Journal of Mammalogy 38: 412.

DIKMANS, G. 1941. A new nematode,

Skrjabinema parva (Nematoda:

Oxyuroidea), from deer. Proceedings of the Helminthological Society of Washington 9: 66-68.

DORAN, D.J. 1954a. A catalogue of the Protozoa and helminths of North American rodents.

I. Protozoa and Acanthocephala. American Midland Naturalist 52: 118-128.

1954b. A catalogue of the Protozoa and helminths of North American rodents. II.

Cestoda. American Midland Naturalist 52: 469-480.

1955a. A catalogue of the Protozoa and

helminths of North American rodents. III.

Nematoda. American Midland Naturalist 53: 162-175.

1955b. A catalogue of the Protozoa and

helminths of North American rodents. IV.

Trematoda. American Midland Naturalist 53: 446-454.

DRAPER, D.J. 1952. Post-mortem examinations of deer shot in Rondeau Provincial Park in January, 1952. Ontario Department of Lands and Forests, Wildlife Research Report 1: 1-3.

DYER, W.G. 1969. A checklist of the Protozoa and helminths of the deer mouse,

Peromyscus maniculatus, in North America. American Midland Naturalist 81: 258-262.

ERICKSON, A.B. 1938. Parasites of some Minnesota Cricitidae and Zapodidae, and a host catalogue of helminth parasites of native American mice. American Midland Naturalist 20: 575-589.

- 1950. Helminths of Minnesota Canidae in relation to food habits, and a host list and key to the species reported from North America. American Midland Naturalist 32 358-372.

FRANK, J.F. 1952. A study of the incidence of trichinosis in swine in the maritime provinces. Canadian Journal of Comparative Medicine 16: 73-77.

FRECHETTE, J.L., AND M. PANISSET. 1973. Contribution a l'etude de l'epizootiologie de la trichinose au Quebec. Canadian Journal of Public Health 64: 443-444.

FYVIE, A. 1969. Manual of common parasites, diseases, and anomalies of wildlife in Ontario. Department of Lands and Forests. $102 \mathrm{pp}$.
GIBBS, H.C. 1964. Observations on an outbreak of clinical parasitism in ewes during the winter months. Canadian Veterinary Journal 5: 8-11.

HOLMES, J.C. 1973. Site selection by parasitic helminths: interspecific interactions, site segregation, and their importance to the development of helminth communities. Canadian Journal of Zoology 51: 333-347.

KINGSCOTE, A.A. 1938. Common parasites of Canadian animals. Canadian Journal of Comparative Medicine 2: 47-55.

KUITUNEN-EKBAUM, E., AND D. WEBSTER. 1947. Trichinosis in wild rats in Toronto. Canadian Journal of Public Health 38: 7678.

LANKESTER, M.W. 1976. A protostrongylid nematode of woodland caribou and implications in moose management. Proceedings of the 12th North American Moose Conference Workshop. St. John's, Newfoundland. 173-190.

- 1984. Pests, parasites and diseases of moose (Alces alces) in North America. In press.

LANKESTER, M.W., AND J.B. SNIDER. 1982. Rumenfilaria andersoni $\mathrm{n}$. gen., n. sp. (Nematoda: Filarioidea) in moose, Alces alces (L.), from northwestern Ontario, Canada. Canadian Journal of Zoology 60: 2455-2458.

LEIBY, P.D., AND W.G. DYER. 1971. Cyclophyllidean tapeworms of wild Carnivora. Parasitic disease of wild mammals. Ed. J.W. Davis and R.C. Anderson. Iowa State University Press, Iowa. 174-234.

MAGATH, T.B. 1954. The importance of sylvatic hydatid disease. Journal of the American Veterinary Medical Association 125: 411414.

NEILAND, K.A., AND C. DUKEMINIER. 1972. A bibliography of the parasites, disease, and disorders of several important wild ruminants of the northern hemisphere. Alaska Department of Fisheries, Game, and Wildlife Technical Bulletin No. 3, 151p.

OLSEN, O.W., AND R. FENSTERMACHER. 1942. Parasites of moose in northern Minnesota. American Journal of Veterinary Research 3: 403-408.

PARNELL, I.W. 1934. Animal parasites of Northeast Canada. Canadian FieldNaturalist 48: 111-115.

PEARSON, J.C. 1951. A species of Alaria from the marten (Martes a. americana). Journal of Parasitology 37 (supplement): 25. 
PYBUS, M.J., AND W.M. SAMUEL. 1984. Attempts to find a laboratory host for Parelaphostrongylus andersoni and Parelaphostrongylus ododoilei (Nematoda: Protostrongylidae). Canadian Journal of Zoology 62: 1181-1184.

- 1984. Parelaphostrongylus andersoni (Nematoda: Protostrongylidae) and $P$. odocoilei in two cervid definitive hosts. Journal of Parasitology 70: 507-515.

RAUSCH, R. L. 1952a. Hydatid disease in boreal regions. Arctic 5: 157-174.

RILEY, W.A. 1939. The need for data relative to the occurrence of hydatids and of Echinococcus granulosus in wildlife. Journal of Wildlife Management 3: 255257.

ROGERS, L.L., AND S.M. ROGERS. 1976. Parasites of bears: a review. Bears - their biology and management. Edited by M.R. Pelton, G.E. Folk, and J.F. Leatfer. IUCN Publication New Series 40. Morges, Switzerland. pp. 411-430.

ROUNTREE, J.L., J.F. WITTER, AND H.L. CHUTE. 1954. Acute lungworm infection. A case report. Veterinary Medicine 49: 306-307.

SAMSON, J., AND J.C. HOLMES. 1985a. The effect of temperature on rates of development of larval Protostrongylus spp. (Nematoda: Metastrongyloidea) from bighorn sheep, Ovis canadensis canadensis in the snail Valbria pulchella. Canadian Journal of Zoology 63: 1445-1448.

1985b. Modes of entry of first stage larvae of Protostrongylus stilesi and P. rushi (Nematoda: Metastrongyloidea) in the snail intermediate host Vallania pulchella. Canadian Journal of Zoology 63: 24812482

SAMUEL, W.M. 1968. Endoparasites of domestic ruminants and white-tailed deer. Report and Transactions of the 33rd North American Wildlife and Natural Resources Conference. 1968.

SAMUEL, W.M., T.R. PLATT, AND S.M. KNISPEL-KRAUSE. 1985. Gastropod intermediate hosts and transmission of Parelaphostrongylus odocoilei, a muscleinhabiting nematode of mule deer, Odocoileus h. hemionus, in Jasper National Park, Alberta. Canadian Journal of Zoology 63: 928-932.

SAUNDERS, B.P. 1974. Prevalence of Parelaphostrongylus tenuis in white-tailed deer in northwestern Ontario related to moose population densities. Journal of
Wildlife Management (PRESS)

SCOTT, J.W. 1941. A new genus and new species of tapeworm from the bighorn sheep. Anatomical Record 81: 65-66.

SOON, B.-K, AND R.S. DORNEY. 1969b. Occurrence of Eimeria tamiasciuri in Ontario red squirrels ( Tamiasciurus hudsonicus). Canadian Journal of Ecology 47: 731-732.

SPENCER, G.J. 1937. The menace of rat parasites in Vancouver in 1936. Entomological Society of B.C. 33: 44-45.

SUKHDEO, M.V.K., AND E. MEEROVITCH. 1977. Comparison of three geographical isolates of Trichinella. Canadian Journal of Zoology 55: 2060-2064.

SWALES, W.E. 1933a. A review of Canadian helminthology. I. The present status of knowledge of the helminth parasites of domesticated and semidomesticated mammals and economically important birds in Canada, as determined from work published prior to 1933. Canadian Journal of Research, Section D, Zoological Sciences 8: 468-477.

SWALES, W.E., AND L. CHOQUETTE. 1940.

Diagnostic et treitement des maladies parasitaires du mouton dans la provice de Quebec. Canadian Journal of Comparative Medicine 4: 292-294.

UHAZY, L.S., AND J.C. HOLMES. 1971 Helminths of the Rocky Mountain bighorn sheep in western Canada. Canadian Journal of Zoology 49: 507-512.

UHAZY, L.S., AND J.L. MAHRT. 1971. Coccidia of Rocky Mountain bighorn sheep in Western Canada. Canadian Journal of Zoology 49: 1461-1464.

WARDLE, R.A. 1933. The parasitic helminths of Canadian animals. I. The Cestodaria and Cestoda. Canadian Journal of Research, Section D, Zoological Sciences 8: 317-333

WEBSTER, C.A., AND T.W.M. CAMERON 1967. Epidemiology and diagnosis of echinococcosis in Canada. Canadian Medical Association Journal 96: 600-607.

WOBESER, G., R.J. CAWTHORN, AND A.A. GAJADHAR. 1983. Pathology of Sarcocystis campestris infection in Richardson's ground squirrels (Spermophilus richardsonii). Canadian Journal of Comparative Medicine 47: 198202.

WOLFGANG, R.W., AND J.B. POOLE. 1956. Distribution of Echinococcus disease in northwestern Canada. American Journal of Tropical Medicine and Hygiene 5: 869-871 


\section{INDEX \\ PARASITES}

Acanthatrium eptesici 11

Acanthatrium nycteridis 11

Acanthocephala 44

Ackertia marmotae 32

Adeleina 7

Adenophorea 25

Aelurostrongylus pridhami 35

Alaria alata 8

Alaria americana 8

Alaria arisaemoides 9

Alaria canadensis 9

Alaria canis

see Alaria americana 8

Alaria craterum 9

Alaria marcianae 9

Alaria mustelae 9

Alaria taxideae 9

Alaria spp. 9

Allintoshius nycticeius 41

Amoebida 3

Amphibiophilidae 39

Ancylostoma caninum 35

Ancylostomatidae 35

Ancylostomatoidea 35

Andersonstrongylus captivensis 35

Andrya arctica 14

Andrya bairdi 15

Andrya communis 15

Andrya macrocephala 15

Andrya neotomae 15

Andrya primordialis 15

Andrya sciuri 15

Andrya sp. 15

Angiostrongylidae 35

Angiostrongylus vasorum 35

Anisakidae 28

Anisakis sp. 28

Anoplocephalidae 14

Apicomplexa 3

Apophallus venustus 11

Apophallus sp. 11

Archiacanthocephala 44

Arthrocephalus lotoris 35

Ascaridida 28

Ascarididae 28

Ascaridoidea 28

Ascaris columnaris see Baylisascaris columnaris 28

Ascaris devosi see Baylisascaris devosi 28

Ascaris obvelata see Syphacia obvelata 30
Ascaris transfuga

see Baylisascaris transfuga 29

Ascaris spp. 28

Atriotaenia procyonis 15

Avitellina arctica 15

Avitellina spp. 15

Baylisascaris columnaris 28

Baylisascaris devosi 28

Baylisascaris laevis 29

Baylisascaris transfuga 29

Baylisascaris sp. 29

Besnoitia spp. 3

Brachylaemidae 8

Brachylaima sp. 8

Capillaria aerophila 26

Capillaria eubursata 26

Capillaria hepatica 26

Capillaria michiganensis 27

Capillaria mustelorum 27

Capillaria plica 27

Capillaria procyonis 27

Capillaria putorii 27

Capillaria ransomia 27

Capillaria spp. 27

Castorstrongylus castoris 39

Catatropis filamentis see Notocotylus filamentis 12

Catenotaenia dendritica 17

Catenotaeniidae 17

Cephaluris alaskensis 30

Cephaluris coloradensis 30

Cercopithifilaria leporinus 32

Cestoidea 14

Chabertiidae 39

Chilomastix bettencourti 3

Choanotaenia nebraskensis 17

Choanotaenia peromysci 17

Choanotaenia spermophili 17

Choanotaenia sp. 17

Citellinema bifurcatum 40

Cittotaenia pectinata 15

Cittotaenia pectinata americana 15

Cladotaenia circi 18

Cladotaenia globifera 18

Cladotaenia mirsoevi 18

Cladotaenia spp. 19

Cooperia oncophora 42

Crenosoma decoratum 36

Crenosoma goblei 36

Crenosomahermani 36 
Crenosoma mephitidis 36

Crenosoma petrowi 36

Crenosoma potos 36

Crenosoma vulpis 36

Crenosoma spp. 36

Crenosomatidae 36

Cryptocotyle lingua 11

Cyclophyllidea 14

Cylicospirura subaequalis 34

Cysticercus fasciolaris 19

Cysticercus ovis 19

Cysticercus pisiformis 19

Cysticercus talpae 19

Cysticercus tarandi 19

Cysticercus tenuicollis 19

Cysticercus spp. 19

Diandrya vancouverensis 15

Dicrocoeliidae 8

Dicrocoelium dendriticum 8

Dicrocoelium lanceolatum see Dicrocoelium dendriticum 8

Dictyocaulidae 39

Dictyocaulus filaria 39

Dictyocaulus hadweni 39

Dictyocaulus viviparus 39

Dictyocaulus spp. 40

Digenea 8

Dilepididae 17

Dioctophyma renale 25

Dioctophymatidae 25

Dioctophymatoidea 25

Dipetalonema mephitis 32

Dipetalonema sprenti 32

Diphyllobothriidae 24

Diphyllobothrium cordatum 24

Diphyllobothrium latum 24

Diphyllobothrium ursi 24

Diphyllobothrium spp. 25

Diplomonadida 2

Diplostomidae 8

Diplostomum mergi 9

Diplostomum spathaceum 9

Dipylidium caninum 17

Dirofilaria scapiceps 32

Dirofilaria ursi 32

Dracunculidae 31

Dracunculoidea 31

Dracunculus insignis 31

Dracunculus lutrae 31

Dracunculus spp. 31

Echinochasmus schwartzi 10

Echinococcus granulosus 19

Echinococcus granulosus canadensis 20

Echinococcus multilocularis 20

Echinococcus multilocularis sibiricensis 20
Echinococcus sp. 20

Echinoparyphium contiguum 10

Echinoparyphium recurvatum 10

Echinoparyphium sp. 10

Echinorhynchida 44

Echinorhynchidae 44

Echinorhynchus lateralis 44

Echinostoma callawayensis 10

Echinostoma revolutum 10

Echinostoma spp. 10

Echinostomatidae 10

Eimeria ahsata 3

Eimeria albertensis 4

Eimeria arizonensis 4

Eimeria athabascensis 4

Eimeria banffensis 4

Eimeria barretti 4

Eimeria bilamellata 4

Eimeria borealis 4

Eimeria calentinei 4

Eimeria callospermophili 4

Eimeria carinii 4

Eimeria circumborealis 4

Eimeria confusa 4

Eimeria crandallis 4

Eimeria dorneyi 4

Eimeria faurei 4

Eimeria granulosa 4

Eimeria holmesi 5

Eimeria intricata 5

Eimeria keithi 5

Eimeria klondikensis 5

Eimeria lateralis 5

Eimeria leporis 5

Eimeria miyairii 5

Eimeria moschati 5

Eimeria mustelae 5

Eimeria ninakohlyakimovae 5

Eimeria ontarioensis 5

Eimeria oomingmakensis 5

Eimeria ovibovis 5

Eimeria ovina 5

Eimeria parva 5

Eimeria princepsis 5

Eimeria robertsoni 6

Eimeria rochesterensis 6

Eimeria rowani 6

Eimeria ruficaudati 6

Eimeria separata 6

Eimeria siniffi 6

Eimeria spermophili 6

Eimeria stiedae 6

Eimeria tamiasciuri 6

Eimeria toddi 6

Eimeria townsendi 6

Eimeria worleyi 6

Eimeria spp. 6 
Eimeriina 3

Elaeophora schneideri 32

Elaphostrongylus cervi 37

Elaphostrongylus odocoilei 37

Encephalitozoon cuniculi 8

Enoplida 25

Entamoeba muris 3

Eucestoda 14

Eucoccidiida 3

Eugenuris talkeetnaeuris see Labiostomum talkeetnaeuris 30

Eumetazoa 8

Euparyphium inerme 10

Euparyphium melis 10

Euryhelmis pyriformis 11

Eustrongylides sp. 25

Fasciola hepatica 10

Fasciola magna see Fascioloides magna 11

Fasciolidae 10

Fascioloides magna 11

Fibricola cratera 10

Fibricola laruei 10

Filaria scapiceps see Dirofilaria scapiceps 32

Filaria sp. 31

Filariidae 31

Filarioidea 31

Filaroides bronchialis see Filaroides martis 36

Filaroides canadensis 36

Filaroides martis 36

Filaroides mephitis 37

Filaroididae 36

Frenkelia microti 6

Giardia mesocricetus 2

Giardia microti 2

Giardia muris 2

Giardia peromysci 2

Giardia simoni 2

Gigantorhynchida 44

Gigantorhynchidae 44

Gnathostoma miyazakii 33

Gnathostomatidae 33

Gnathostomatoidea 33

Graphidiella ochotonae 39

Haemonchus contortus 42

Haemonchus ostertagi 42

Haemonchus placei 42

Heligmosomidae 40

Heligmosomoides polygyrus 40

Heligmosomum carolinensis 40

Heligmosomum hudsonius 40

Heligmosomum muris 40
Hepatozoon spp. 7

Heterakidae 29

Heterakoidea 29

Heterakis spumosa 29

Heterophyidae 11

Heteroxynema cucullatum 30

Heteroxynematidae 29

Hexamitidae 2

Hymenolepididae 17

Hymenolepis bennetti 17

Hymenolepis citelli 17

Hymenolepis diminuta 17

Hymenolepis evaginata 17

Hymenolepis gertschi 17

Hymenolepis horrida 18

Hymenolepis johnsoni 18

Hymenolepis murina 18

Hymenolepis octocoronata 18

Hymenolepis spp. 18

\section{Isospora marquardti 7}

Isospora yukonensis 7

Kinetoplastida 2

Labiostomum coloradensis 30

Labiostomum rauschi 30

Labiostomum talkeetnaeuris 30

Labiostomum sp. 30

Lecithodendriidae 11

Lecithodendrium anticum 11

Lecithodendrium posticum 11

Limatulum gastroides 11

Litomosoides carinii 32

Litomosoides guiterasi 32

Lobosea 3

Marshallagia marshalli 42

Marshallagia spp. 42

Mastigophora 2

Mastophorus muris see Protospirura muris 35

Mediorhynchus robustus 44

Mesocestoides kirbyi 18

Mesocestoides latus 18

Mesocestoides spp. 18

Mesocestoididae 18

Metastrongyloidea 35

Metorchis conjunctus 12

Microfilaria mephitis see Dipetalonema mephitis 32

Microphallidae 12

Microphallus sp. 12

Microspora 8

Microsporea 8

Microsporida 8

Molineidae 40 
Molineus sp. 40

Moniezia benedini 15

Moniezia expansa 16

Moniezia sp. 16

Moniliformida 44

Moniliformidae 44

Moniliformis spiradentatis 44

Multiceps multiceps

see Taenia multiceps 22

Multiceps serialis see Taenia serialis 23

Multiceps sp. 21

Murielus harpespiculus 40

Nanophyetus salmincola 14

Nemathelminthes 25

Nematoda 25

Nematodirella alcidis 40

Nematodirella longispiculata 40

Nematodirella longispiculata antilocaprae 41

Nematodirella sp. 41

Nematodirus archari 41

Nematodirus davtiani 41

Nematodirus filicollis 41

Nematodirus helvetianus 41

Nematodirus maculosus 41

Nematodirus odocoilei 41

Nematodirus oiratianus 41

Nematodirus spathiger 41

Nematodirus triangularis 41

Nematodirus spp. 41

Nematospiroides carolinensis see Heligmosomoides carolinensis 40

Nematospiroides dubius see Heligmosomoides polygyrus 40

Nippostrongylus muris see Heligmosomoides muris 40

Notocotylidae 12

Notocotylus filamentis 12

Notocotylus quinqueserialis see Quinqueserialis quinqueserialis 12

Notocotylus urbanensis 12

Nudacotyle novicia 12

Obeliscoides cuniculi 42

Obeliscoides cuniculi cuniculi 42

Obeliscoides cuniculi multistriatus 42

Oesophagostomum venulosum 39

Oesophagostomum sp. 39

Onchocerca cervipedis 33

Onchocerca sp. 33

Onchocercidae 32

Oochoristica oklahomensis 16

Opisthorchiidae 12

Opisthorchis tonkae 12

Ornithostrongylidae 41

Orthostrongylus macrotis 37
Oslerus osleri 37

Oslerus sp. 37

Ostertagia bisonis 42

Ostertagia circumcincta 42

Ostertagia dikmansi 43

Ostertagia marshalli 43

Ostertagia mossi 43

Ostertagia occidentalis 43

Ostertagia odocoilei 43

Ostertagia oreamni 43

Ostertagia ostertagi 43

Ostertagia trifurcata 43

Ostertagia spp. 43

Oxynema sp. 29

Oxyurida 29

Oxyuridae 30

Oxyuroidea 29

Palaeacanthocephala 44

Paragonimus kellicotti 14

Paragonimus spp. 14

Parametorchis canadensis 13

Paramphistomidae 13

Paramphistomum cervi 13

Paramphistomum liorchis 13

Paramphistomum spp. 13

Paranoplocephala infrequens 16

Paranoplocephala lemmi 16

Paranoplocephala variabilis 16

Parelaphostongylus andersoni 37

Parelaphostongylus odocoilei 37

Parelaphostongylus tenuis 37

Parelaphostongylus sp. 38

Paruterina candelabraria 17

Paruterina rauschi 17

Passalurus nonanulatus 30

Pelodera strongyloides 31

Phagicola longa 11

Pharyngostomoides adenocephala 10

Pharyngostomoides procyonis 10

Physaloptera bispiculata 33

Physaloptera maxillaris 33

Physaloptera praeputialis 33

Physaloptera rara 34

Physaloptera spinicauda 34

Physaloptera spp. 34

Physalopteridae 33

Physalopteroidea 33

Placoconus lotoris 35

Plagiorchiidae 13

Plagiorchis elegans 13

Plagiorchis muris 13

Plagiorchis noblei 13

Plagiorchis proximus 13

Plagiorchis vespertilionis 14

Plagiorchis sp. 14

Platyhelminthes 8 
Pneumostrongylus tenuis see Parelaphostrongylus tenuis 37

Polymorphida 44

Polymorphidae 44

Polymorphus paradoxus 44

Polymorphus spp. 44

Postharmostomum helicis 8

Prosthodendrium macnabi 11

Prosthodendrium naviculum 11

Prosthodendrium silvai 12

Prosthodendrium swansoni 12

Prosthodendrium spp. 12

Protospirura ascaroidea 34

Protospirura muris 35

Protostrongylidae 37

Protostrongylus boughtoni 38

Prostostrongylus macrotis 38

Prostostrongylus rushi 38

Protostrongylus stilesi 38

Protostrongylus sp. 38

Protozoa 2

Pseudophyllidea 24

Psilostomidae 14

Psilostomum ondatrae 14

Quinqueserialis quinqueserialis 12

Quinqueserialis zibethicai 12

Retortamonadida 3

Rhabditida 31

Rhabditidae 31

Rhabditoidea 31

Rictulariidae 34

Rictularioidea 34

Rictularia citelli 34

Rictularia coloradensis 34

Rictularia lucifugus 34

Rictularia sp. 34

Rumenfilaria andersoni 33

Sarcocystis campestris 7

Sarcocystis spp. 7

Sarcodina 3

Sarcomastigophora 2

Scaphiostomum pancreaticum 8

Schistocephalus solidus 25

Schistosomatidae 14

Schistosomatium douthitti 14

Schizorchis caballeroi 16

Schizorchis ochotonae 16

Secernentea 28

Setaria cervi 33

Setaria labiatopapillosa 33

Setaria tundra 33

Setaria yehi 33

Setaria spp. 33
Skrjabinema oreamni

see Ostertagia oreamni 43

Skrjabinema ovis 30

Skrjabinema sp. 30

Skrjabingylidae 38

Skrjabingylus chitwoodorum 38

Skrjabingylus lutrae 38

Skrjabingylus magnus 38

Skrjabingylus nasicola 39

Skrjabingylus sp. 39

Sobolevingylus sp. 36

Spiculopteragia bohmi 43

Spirocerca arctica 34

Spirocerca lupi 34

Spirocercidae 34

Spirurida 31

Spiruridae 34

Spiruroidea 34

Spirura infundibuliformis 35

Sporozoea 3

Stephanoproraoides lawi 14

Stichorchis subtriquetrus 13

Strongylida 35

Strongyloidea 39

Strongyloididae 31

Strongyloides papillosus 31

Strongyloides ratti 31

Strongyloides robustus 31

Strongyloides sp. 31

Subuluridae 29

Subuluroidea 29

Syphacia citelli 30

Syphacia eutamii 30

Syphacia obvelata 30

Syphacia peromysci 30

Syphacia thompsoni 30

Taeniidae 18

Taenia crassiceps 21

Taenia hydatigena 21

Taenia krabbei 21

Taenia laticollis 22

Taenia macrocystis 22

Taenia martis 22

Taenia multiceps 22

Taenia mustelae 22

Taenia omissa 23

Taenia ovis 23

Taenia pisiformis 23

Taenia polyacantha 23

Taenia rileyi 23

Taenia serialis 23

Taenia sibirica 23

Taenia taeniaeformis 24

Taenia taxidiensis 24

Taenia tenuicollis

see Taenia mustelae 22 
Taenia twitchelli 24

Taenia spp. 24

Teladorsagia davtiani 43

Teladorsagia spp. 43

Thysanosoma actinioides 16

Toxascaris leonina 29

Toxascaris limbata see Toxascaris leonina 29

Toxocara canis 29

Toxocara cati 29

Toxocara mystax see Toxocara cati 29

Toxoplasma gondii 7

Travassosius americanus 44

Trematoda 8

Trichinellidae 25

Trichinelloidea 25

Trichinella spiralis 25

Trichinella spiralis var. pseudospiralis 26

Trichinella spp. 26

Trichomonadida 3

Trichomonas muris 3

Trichosomoides crassicauda 27

Trichostrongylidae 42

Trichostrongyloidea 39

Trichostrongylus axei 44

Trichostrongylus calcaratus 44

Trichostrongylus longispicularis 44

Trichostrongylus spp. 44

Trichuridae 26

Trichuris fossor 27

Trichuris leporis 27

Trichuris muris 27

Trichuris opaca 28

Trichuris oreamnos 28

Trichuris ovis 28

Trichuris schumakovitschi 28

Trichuris vulpis 28

Trichuris spp. 28

Trilobostrongylus bioccai 36

Troglotrematidae 14

Troglostrongylus wilsoni 36

Trypanosoma citelli 2

Trypanosoma evotomys 2

Trypanosoma hedricki 2

Trypanosoma leporis-sylvaticus 2

Trypanosoma lewisi 2

Trypanosoma microti 3

Trypanosoma myoti 3

Trypanosoma otospermophili 3

Trypanosoma peromysci 3

Trypanosoma soricis 3

Trypanosoma tamiasi 3

Trypanosoma sp. 3

Uncinaria yukonensis 35
Varestrongylus alpenae 38

Wardius zibethicus 13

Warrenius bifurcatus see Citellinema bifurcatum 40

Wehrdikmansia cervipedis see Onchocerca cervipedis 33

Wyominia tetoni 16

Zoomastigophorea 2

Zygocotyle lunata 13 
Alces alces 48

Alces alces americana 48

Alces alces andersoni 49

Alces americana see Alces alces 48

Alopex lagopus 51

Antilocapridae 47

Antilocapra americana 47

Artiodactyla 47

Barren-ground caribou 50

Bison bison 47

Blarina brevicauda 56

Bovidae 47

Canidae 51

Canis latrans 51

Canis lupus 51

Carnivora 51

Castoridae 58

Castor canadensis 58

Castor canadensis leucodontus 58

Cavia porcellus 58

Caviidae 58

Cervidae 48

Cervus canadensis see Cervus elaphus 49

Cervus canadensis nelsoni see Cervus elaphus 49

Cervus elaphus 49

Chiroptera 55

Citellus franklinii see Spermophilus franklinii 62

Citellus richardsonii see Spermophilus richardsonii 62

Citellus tridecemlineatus see Spermophilus tridecemlineatus 63

Clethrionomys gapperi 58

Clethrionomys gapperi gapperi 58

Clethrionomys gapperi saturatus 58

Clethrionomys sp. 58

Clethrionomyx gapperi see Clethrionomys gapperi 58

Cottontail 57

Coyote $\mathrm{x}$ Dog 52

\section{Deer 50}

Dicrostonyx groenlandicus 58

Dicrostonyx groenlandicus richardsoni 58

Dicrostonyx hudsonius 58

Dicrostonyx torquatus 58

Didelphidae 47
Didelphis marsupialis see Didelphis virginiana 47

Didelphis virginiana 47

Elk 51

Eptesicus fuscus 55

Erethizontidae 58

Erethizon dorsatum 58

Euarctos americanus americanus see Ursus americanus 55

Eutamias amoenus 62

Eutamias minimus 62

Eutheria 47

Evotomys saturatus see Myotis saturatus 56

Felidae 52

Felis canadensis see Lynx canadensis 52

Felis concolor 52

Felis concolor vancouverensis 52

Felis lynx see Lynx canadensis 52

Felis rufus see Lynx rufus 52

Ferret 54

Field mice 61

Geomyidae 58

Glaucomys sabrinus 62

Gulo gulo 52

Hamster (lab.) 61

Insectivora 56

Lagomorpha 56

Lasionycteris noctivagans 56

Lasiurus cinereus 56

Lemmus trimucronatus see Lemmus sibiricus 59

Lemmus trimucronatus trimucronatus see Lemmus sibiricus trimucronatus 59

Leporidae 56

Lepus americanus 56

Lepus americanus americanus 57

Lepus americanus pallidus 57

Lepus americanus struthopus 57

Lepus capensis 57

Lepus cuniculus see Oryctolagus cuniculus 57

Lepus sylvaticus see Sylvilagus nuttallii 57 
Lontra canadensis

see Lutra canadensis 53

Lutra canadensis 53

Lutra canadensis canadensis 53

Lynx canadensis 52

Lynx lynx

see Lynx canadensis 52

Lynx rufus 52

Lynx rufus fasciatus 52

Mammalia 47

Marmota monax 62

Marmota monax canadensis 62

Marmota vancouverensis 62

Marsupialia 47

Martes americana 53

Martes americana actuosa 53

Martes americana americana 53

Martes americanus see Martes americana 53

Martes martes 53

Martes pennanti 53

Martes pennanti pennanti 53

Mephitis mephitis 53

Meriones unguiculatus 59

Mesocricetus auratus (Lab) 59

Metatheria 47

Microtus chrotorrhinus 59

Microtus ochrogaster 59

Microtus pennsylvanicus 59

Microtus pennsylvanicus drummondii 59

Microtus pennsylvanicus pennsylvanicus 59

Microtus pennsylvanicus terraenovae 59

Microtus sp. 59

Mink 54

Moose 51

Mouse (Lab) 61

Muridae 58

Mus musculus 59

Mus musculus (lab) 59

Muskrat 61

Mustelidae 52

Mustela cicognani anguinae see Mustela erminea anguinae 54

Mustela erminea 54

Mustela erminea anguinae 54

Mustela erminea richardsonii 54

Mustela frenata 54

Mustela frenata longicauda 54

Mustela putorius (Lab) 54

Mustela vison 54

Mustela vison vison 54

Myotis californicus 56

Myotis lucifugus 56

Myotis saturatus see Myotis yumanensis saturatus 56
Myotis volans longicrus 56

Myotis yumanensis saturatus 56

Napaeozapus insignis algonquinensis see Napaeozapus insignis insignis 63

Napaeozapus insignis insignis 63

Neotoma cinerea 60

Ochotona collaris 57

Ochotona princeps 57

Ochotonidae 57

Odocoileus columbianus see Odocoileus hemionus columbianus 49

Odocoileus hemionus 49

Odocoileus hemionus columbianus 49

Odocoileus hemionus hemionus 49

Odocoileus virginianus 49

Odocoileus virginianus borealis 50

Odocoileus virginianus dacotensis 50

Odocoileus virginianus ochrourus 50

Odocoileus sp. 50

Ondatra zibethica see Ondatra zibethicus 60

Ondatra zibethicus 60

Ondatra zibethicus obscurus 60

Ondatra zibethicus osoyoosensis 60

Otter 55

Oreamnos americanus 47

Oryctolagus cuniculus 57

Ovibos moschatus 47

Ovis canadensis 47

Ovis canadensis californiana 48

Ovis canadensis californianus see Ovis canadensis californiana 48

Ovis canadensis canadensis 48

Ovis dalli 48

Pedomys ochrogaster see Microtus ochrogaster 59

Peromyscus leucopus 60

Peromyscus leucopus noveboracensis 60

Peromyscus maniculatus 60

Peromyscus maniculatus gracilis 61

Peromyscus maniculatus nebrascensis 61

Phenacomys intermedius 61

Procyonidae 55

Procyon lotor 55

Procyon lotor hirtus 55

Rabbit 57

Rabbit (lab) 57

Raccoon 55

Rangifer arcticus see Rangifer tarandus 50

Rangifer anticus arcticus see Rangifer tarandus 50 
Rangifer carbibou sylvestris see Rangifer tarandus 50

Rangifer montanus see Rangifer tarandus 50

Rangifer tarandus 50

Rangifer tarandus caribou 50

Rangifer tarandus groenlandicus 50

Rangifer tarandus tarandus $\mathbf{5 0}$

Rangifer tarandus terraenovae 50

Rangifer sp. 50

Rat (lab) 61

Rattus norvegicus 61

Rattus sp. 61

Red fox 52

Reindeer 51

Rodentia 58

Sciuridae 62

Sciurus carolinensis 62

Sigmodon hispidus (lab) 61

Soricidae 56

Sorex cinereus 56

Sorex fumeus 56

Sorex vagrans 56

Spermophilus columbianus 62

Spermophilus franklinii 62

Spermophilus parryii 62

Spermophilus richardsonii 62

Spermophilus tridecemlineatus 63

Spermophilus undulatus plesius

see Spermophilus parryii 62

Spilogale gracilis olympica

see Spilogale putorius gracilis 54

Spilogale putorius gracilis 54

Squirrel 63

Sylvilagus floridanus 57

Sylvilagus nuttallii 57

Synaptomys borealis 61

Synaptomys cooperi cooperi 61

Tamias striatus 63

Tamiasciurus hudsonicus 63

Tamiasciurus hudsonicus hudsonicus 63

Taxidea taxus 54

Thalarctos maritimus see Ursus maritimus 55

Thomomys talpoides 58

Ursidae 55

Ursus americanus 55

Ursus americanus americanus 55

Ursus americanus vancouveri 55

Ursus arctos 55

Ursus maritimus 55

Varying hare 58

Vespertilionidae 55
Vespertilio subtilus see Myotis lucifugus 56

Vulpes fulva see Vulpes vulpes 52

Vulpes vulpes 52

Zapodidae 63

Zapus hudsonius 63 

